Alexander Premet

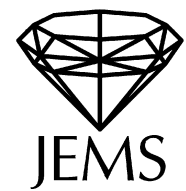

\title{
Enveloping algebras of Slodowy slices and the Joseph ideal
}

Received June 17, 2005, and in revised form March 30, 2006

\begin{abstract}
Let $G$ be a simple algebraic group over an algebraically closed field $\mathbb{k}$ of characteristic 0 , and $\mathfrak{g}=$ Lie $G$. Let $(e, h, f)$ be an $\mathfrak{s l}_{2}$-triple in $\mathfrak{g}$ with $e$ being a long root vector in $\mathfrak{g}$. Let $(\cdot, \cdot)$ be the $G$-invariant bilinear form on $\mathfrak{g}$ with $(e, f)=1$ and let $\chi \in \mathfrak{g}^{*}$ be such that $\chi(x)=(e, x)$ for all $x \in \mathfrak{g}$. Let $\mathcal{S}$ be the Slodowy slice at $e$ through the adjoint orbit of $e$ and let $H$ be the enveloping algebra of $\mathcal{S}$; see [31]. In this article we give an explicit presentation of $H$ by generators and relations. As a consequence we deduce that $H$ contains an ideal of codimension 1 which is unique if $\mathfrak{g}$ is not of type A. Applying Skryabin's equivalence of categories we then construct an explicit Whittaker model for the Joseph ideal of $U(\mathfrak{g})$. Inspired by Joseph's Preparation Theorem we prove that there exists a homeomorphism between the primitive spectrum of $H$ and the spectrum of all primitive ideals of infinite codimension in $U(\mathfrak{g})$ which respects Goldie rank and GelfandKirillov dimension. We study highest weight modules for the algebra $H$ and apply earlier results of Miličić-Soergel and Backelin to express the composition multiplicities of the Verma modules for $H$ in terms of some inverse parabolic Kazhdan-Lusztig polynomials. Our results confirm in the minimal nilpotent case the de Vos-van Driel conjecture on composition multiplicities of Verma modules for finite $\mathcal{W}$-algebras. We also obtain some general results on the enveloping algebras of Slodowy slices and determine the associated varieties of related primitive ideals of $U(\mathfrak{g})$. A sequel to this paper will treat modular aspects of this theory.
\end{abstract}

\section{Introduction}

1.1. Let $\mathbb{k}$ be an algebraically closed field of characteristic 0 and let $G$ be a simple algebraic group over $\mathbb{k}$. Let $\mathfrak{g}=$ Lie $G$ and let $(e, h, f)$ be an $\mathfrak{s l}_{2}$-triple in $\mathfrak{g}$. Let $(\cdot, \cdot)$ be the $G$-invariant bilinear form on $\mathfrak{g}$ with $(e, f)=1$ and define $\chi=\chi_{e} \in \mathfrak{g}^{*}$ by setting $\chi(x)=(e, x)$ for all $x \in \mathfrak{g}$. Let $\mathcal{O}_{\chi}$ denote the coadjoint orbit of $\chi$.

Let $\mathcal{S}_{e}=e+\operatorname{Ker}$ ad $f$ be the Slodowy slice at $e$ through the adjoint orbit of $e$ and let $H_{\chi}$ be the enveloping algebra of $\mathcal{S}_{e}$; see $[31,10,5]$. Recall that $H_{\chi}=\operatorname{End}_{\mathfrak{g}}\left(Q_{\chi}\right)^{\text {op }}$ where $Q_{\chi}$ is a generalised Gelfand-Graev module for $U(\mathfrak{g})$ associated with the $\mathfrak{s l}_{2}$-triple $(e, h, f)$; see $[22,29]$. The module $Q_{\chi}$ is induced from a one-dimensional module $\mathbb{k}_{\chi}$ over a nilpotent subalgebra $\mathfrak{m}_{\chi}$ of $\mathfrak{g}$ such that $\operatorname{dim} \mathfrak{m}_{\chi}=\frac{1}{2} \operatorname{dim} \mathcal{O}_{\chi}$. The subalgebra $\mathfrak{m}_{\chi}$ is $(\operatorname{ad} h)$-stable, all weights of $\operatorname{ad} h$ on $\mathfrak{m}_{\chi}$ are negative, and $\chi$ vanishes on the derived

A. Premet: Department of Mathematics, The University of Manchester, Oxford Road, Manchester M13 9PL, UK; e-mail: sashap@ma.man.ac.uk

Mathematics Subject Classification (2000): Primary 17B35; Secondary 17B63, 17B81 
subalgebra of $\mathfrak{m}_{\chi}$. The action of $\mathfrak{m}_{\chi}$ on $\mathbb{k}_{\chi}=\mathbb{k} 1_{\chi}$ is given by $x\left(1_{\chi}\right)=\chi(x) 1_{\chi}$ for all $x \in \mathfrak{m}_{\chi} ;$ see 2.1 for more details.

Let $\mathfrak{z}_{\chi}$ denote the stabiliser of $\chi$ in $\mathfrak{g}$. Clearly, $\mathfrak{z}_{\chi}$ coincides with the centraliser $\mathfrak{c}_{\mathfrak{g}}(e)$ of $e$ in $\mathfrak{g}$. The subalgebra $\mathfrak{z}_{\chi}$ is $(\operatorname{ad} h)$-stable and it follows from the $\mathfrak{s l}_{2}$-theory that all weights of ad $h$ on $\mathfrak{z}_{\chi}$ are nonnegative integers. Let $x_{1}, \ldots, x_{r}$ be a basis of $\mathfrak{z}_{\chi}$ such that $\left[h, x_{i}\right]=$ $n_{i} x_{i}$ for some $n_{i} \in \mathbb{Z}_{+}$. By [31, Theorem 4.6], to each basis vector $x_{i}$ one can attach an element $\Theta_{x_{i}} \in H_{\chi}$ in such a way that the monomials $\Theta_{x_{1}}^{i_{1}} \ldots \Theta_{x_{r}}^{i_{r}}$ with $\left(i_{1}, \ldots, i_{r}\right) \in \mathbb{Z}_{+}^{r}$ form a basis of $H_{\chi}$ over $\mathbb{k}$. We say that the monomial $\Theta_{x_{1}}^{a_{1}} \ldots \Theta_{x_{r}}^{a_{r}}$ has Kazhdan degree $\sum_{i=1}^{r} a_{i}\left(n_{i}+2\right)$ and denote by $H_{\chi}^{k}$ the span of all monomials as above of Kazhdan degree $\leq k$. According to [31, (4.6)], we then have $H_{\chi}=\bigcup_{k \geq 0} H_{\chi}^{k}$ and $H_{\chi}^{i} \cdot H_{\chi}^{j} \subseteq H_{\chi}^{i+j}$ for all $i, j \in \mathbb{Z}_{+}$. In other words, $\left\{H_{\chi}^{k} \mid k \in \mathbb{Z}_{+}\right\}$is an increasing filtration of the algebra $H_{\chi}$. We call it the Kazhdan filtration of $H_{\chi}$. The corresponding graded algebra gr $H_{\chi}$ is a polynomial algebra in $\operatorname{gr} \Theta_{x_{1}}, \ldots, \operatorname{gr} \Theta_{x_{r}}$ which identifies naturally with the function algebra $\mathbb{k}\left[\mathcal{S}_{e}\right]$ on the special transverse slice $\mathcal{S}_{e}=e+\operatorname{Ker}$ ad $f$ endowed with its Slodowy grading.

According to [31, Theorem 4.6(iv)],

$$
\left[\Theta_{x_{i}}, \Theta_{x_{j}}\right]=\Theta_{x_{j}} \circ \Theta_{x_{i}}-\Theta_{x_{i}} \circ \Theta_{x_{j}} \equiv \Theta_{\left[x_{i}, x_{j}\right]}+q_{i j}\left(\Theta_{1}, \ldots, \Theta_{r}\right)\left(\bmod H_{\chi}^{n_{i}+n_{j}}\right)
$$

where $q_{i j}$ is a polynomial in $r$ variables with initial form of total degree $\geq 2$. Using this result we prove in 2.3 that there exists an associative $\mathbb{k}[t]$-algebra $\mathcal{H}_{\chi}$ free as a module over $\mathbb{k}[t]$ and such that

$$
\mathcal{H}_{\chi} /(t-\lambda) \mathcal{H}_{\chi} \cong \begin{cases}H_{\chi} & \text { if } \lambda \neq 0 \\ U\left(\mathfrak{z}_{\chi}\right) & \text { if } \lambda=0\end{cases}
$$

as $\mathbb{k}$-algebras. Thus $H_{\chi}$ is a deformation of the universal enveloping algebra $U\left(\mathfrak{z}_{\chi}\right)$.

We have a certain degree of freedom in our choice of PBW generators $\Theta_{x_{i}}$. In 2.2 we show that they can be chosen such that the map $\Theta_{x_{i}} \mapsto(-1)^{n_{i}} \Theta_{x_{i}}, 1 \leq i \leq r$, extends to an automorphism of the algebra $H_{\chi}$. This automorphism, denoted by $\sigma$, will play an important rôle later on.

1.2. Given $x \in \mathfrak{g}$ we denote by $Z_{G}(x)$ the centraliser of $x$ in $G$. It is well-known that $C(e):=Z_{G}(h) \cap Z_{G}(e)$ is a Levi subgroup of $Z_{G}(e)$ and the centraliser $Z_{G}(e)$ decomposes as a semidirect product of $C(e)$ and the unipotent radical $R_{u}\left(Z_{G}(e)\right)$. For $i \in \mathbb{Z}_{+}$ put $\mathfrak{z}_{\chi}(i)=\left\{x \in \mathfrak{z}_{\chi} \mid[h, x]=i x\right\}$. It is well-known that $\mathfrak{z}_{\chi}(0)=$ Lie $C(e)$. Clearly, the group $C(e)$ preserves each subspace $\mathfrak{z}_{\chi}(i)$ of $\mathfrak{z}_{x}$.

In [10], Gan and Ginzburg have found a different realisation of the algebra $H_{\chi}$ which enables one to observe that the reductive group $C(e)$ acts on $H_{\chi}$ as algebra automorphisms. Moreover, this action of $C(e)$ preserves the Kazhdan filtration of $H_{\chi}$; see 2.1 for more details. In Section 2 we show that there exists an injective $C(e)$-equivariant linear map $\Theta: \mathfrak{z}_{\chi} \rightarrow H_{\chi}, x \mapsto \Theta_{\chi}$, whose image generates $H_{\chi}$ as an algebra, such that $\operatorname{gr} \Theta\left(\mathfrak{z}_{\chi}\right) \cong \mathfrak{z}_{x}$ as graded $C(e)$-modules. The subspace $\Theta\left(\mathfrak{z}_{\chi}(0)\right)$ can be chosen to be a 
Lie subalgebra of $H_{\chi}$ with respect to the commutator product in $H_{\chi}$. It follows that the map $\Theta$ can be selected in such a way that

$$
\left[\Theta_{x}, \Theta_{y}\right]=\Theta_{[x, y]} \quad(\forall x \in \mathfrak{z} \chi(0), \forall y \in \mathfrak{z} \chi) .
$$

This is a consequence of Lemma 2.4 which states, in particular, that the Lie algebra homomorphism ad $\circ \Theta: \mathfrak{z}_{\chi}(0) \rightarrow \operatorname{Der}\left(H_{\chi}\right)$ coincides with the differential of the locally finite (rational) action of $C(e)$ on $H_{\chi}$. Combined with Lemma 2.1 and Weyl's theorem on complete reducibility this implies that every two-sided ideal of $H_{\chi}$ is $\sigma$-stable; see Corollary 2.1 .

1.3. For $\chi=(e, \cdot)$ we let $\mathcal{C}_{\chi}$ denote the category of all $\mathfrak{g}$-modules on which $x-\chi(x)$ acts locally nilpotently for all $x \in \mathfrak{m}_{\chi}$. Given a $\mathfrak{g}$-module $M$ we set

$$
\mathrm{Wh}(M):=\left\{m \in M \mid x . m=\chi(x) m\left(\forall x \in \mathfrak{m}_{\chi}\right)\right\} .
$$

It should be mentioned here that the algebra $H_{\chi}$ acts on $\mathrm{Wh}(M)$ via a canonical isomorphism $H_{\chi} \cong\left(U(\mathfrak{g}) / N_{\chi}\right)^{\text {ad } \mathfrak{m}_{\chi}}$ where $N_{\chi}$ denotes the left ideal of $U(\mathfrak{g})$ generated by all $x-\chi(x)$ with $x \in \mathfrak{m}_{\chi}$. In the Appendix to [31], Skryabin proved that the functors $V \rightsquigarrow Q_{\chi} \otimes_{H_{\chi}} V$ and $M \rightsquigarrow \mathrm{Wh}(M)$ are mutually inverse equivalences between the category of all $H_{\chi}$-modules and the category $\mathcal{C}_{\chi}$; see also [10, Theorem 6.1].

Skryabin's equivalence implies that for any irreducible $H_{\chi}$-module $V$ the annihilator $\operatorname{Ann}_{U(\mathfrak{g})}\left(Q_{\chi} \otimes_{H_{\chi}} V\right)$ is a primitive ideal of $U(\mathfrak{g})$. By the Irreducibility Theorem, the associated variety $\mathcal{V A}(\mathcal{I})$ of any primitive ideal $\mathcal{I}$ of $U(\mathfrak{g})$ is the closure of a nilpotent orbit in $\mathfrak{g}^{*}$. Generalising a classical result of Kostant on Whittaker modules we show in Section 3 that for any irreducible $H_{\chi}$-module $V$ the associated variety of $\operatorname{Ann}_{U(\mathfrak{g})}\left(Q_{\chi} \otimes_{H_{\chi}} V\right)$ contains the coadjoint orbit $\mathcal{O}_{\chi}$. In the most interesting case where $V$ is a finite-dimensional irreducible $H_{\chi}$-module we prove that

$$
\mathcal{V A}\left(\operatorname{Ann}_{U(\mathfrak{g})}\left(Q_{\chi} \otimes_{H_{\chi}} V\right)\right)=\overline{\mathcal{O}}_{\chi} \quad \text { and } \quad \operatorname{Dim}\left(Q_{\chi} \otimes_{H_{\chi}} V\right)=\frac{1}{2} \operatorname{dim} \overline{\mathcal{O}}_{\chi}
$$

where $\operatorname{Dim}(M)$ is the Gelfand-Kirillov dimension of a finitely generated $U(\mathfrak{g})$-module $M$. In particular, this implies that for any irreducible finite-dimensional $H_{\chi}$-module $V$ the irreducible $U(\mathfrak{g})$-module $Q_{\chi} \otimes_{H_{\chi}} V$ is holonomic.

1.4. Let $\mathfrak{h}$ be a Cartan subalgebra of $\mathfrak{g}$, and let $\Phi$ be the root system of $\mathfrak{g}$ relative to $\mathfrak{h}$. Let $\Pi=\left\{\alpha_{1}, \ldots, \alpha_{\ell}\right\}$ be a basis of simple roots in $\Phi$ with the elements in $\Pi$ numbered as in [4], and let $\Phi^{+}$be the positive system of $\Phi$ relative to $\Pi$. If $\mathfrak{g}$ is not of type A or $\mathrm{C}$, there is a unique long root in $\Pi$ linked with the lowest root $-\widetilde{\alpha}$ on the extended Dynkin diagram of $\mathfrak{g}$; we call it $\beta$. For $\mathfrak{g}$ of type $A_{n}$ and $C_{n}$ we set $\beta=\alpha_{n}$. Choose root vectors $e_{\beta}, e_{-\beta} \in \mathfrak{g}$ corresponding to roots $\beta$ and $-\beta$ such that $\left(e_{\beta},\left[e_{\beta}, e_{-\beta}\right], e_{-\beta}\right)$ is an $\mathfrak{s l}_{2}$-triple and put $h_{\beta}=\left[e_{\beta}, e_{-\beta}\right]$.

In this article we investigate the algebra $H_{\chi}$ in the case where $(e, h, f)=$ $\left(e_{\beta}, h_{\beta}, e_{-\beta}\right)$. Then $\mathcal{O}_{\chi}=\mathcal{O}_{\min }$, the minimal nonzero nilpotent orbit in $\mathfrak{g}^{*}$. We let $H$ denote the minimal nilpotent algebra $H_{\chi}$. One of our main objectives is to give a presentation of $H$ by generators and relations. 
The action of the inner derivation ad $h$ gives $\mathfrak{g}$ a short $\mathbb{Z}$-grading

$$
\mathfrak{g}=\mathfrak{g}(-2) \oplus \mathfrak{g}(-1) \oplus \mathfrak{g}(0) \oplus \mathfrak{g}(1) \oplus \mathfrak{g}(2), \quad \mathfrak{g}(i)=\{x \in \mathfrak{g} \mid[h, x]=i x\}
$$

with $\mathfrak{g}(1) \oplus \mathfrak{g}(2)$ and $\mathfrak{g}(-1) \oplus \mathfrak{g}(-2)$ being Heisenberg Lie algebras. One knows of course that $\mathfrak{g}( \pm 2)$ is spanned by $e_{ \pm \beta}$, that $\mathfrak{z} \chi(i)=\mathfrak{g}(i)$ for $i=1,2$, and that $\mathfrak{z} \chi(0)$ coincides with the image of the Lie algebra homomorphism

$$
\sharp: \mathfrak{g}(0) \rightarrow \mathfrak{g}(0), \quad x \mapsto x-\frac{1}{2}(x, h) h,
$$

whose kernel $\mathbb{k} h$ is a central ideal of $\mathfrak{g}(0)$. The graded component $\mathfrak{g}(-1)$ has a basis $z_{1}, \ldots, z_{s}, z_{s+1}, \ldots, z_{2 s}$ such that the $z_{i}$ 's with $1 \leq i \leq s$ (resp. $s+1 \leq i \leq 2 s$ ) are root vectors for $\mathfrak{h}$ corresponding to negative (resp. positive) roots, and

$$
\left[z_{i}, z_{j}\right]=\left[z_{i+s}, z_{j+s}\right]=0, \quad\left[z_{i+s}, z_{j}\right]=\delta_{i j} f \quad(1 \leq i, j \leq s) .
$$

Moreover, in the present case we can choose $\mathfrak{m}_{\chi}$ to be the span of $f$ and the $z_{i}$ 's with $s+1 \leq i \leq 2 s$, an abelian subalgebra of $\mathfrak{g}$ of dimension $s+1=\frac{1}{2} \operatorname{dim} \mathcal{O}_{\min }$. We set $z_{i}^{*}:=z_{i+s}$ for $1 \leq i \leq s$ and $z_{i}^{*}:=-z_{i-s}$ for $s+1 \leq i \leq 2 s$.

Let $C$ denote the Casimir element of $U(\mathfrak{g})$ corresponding to the bilinear form $(\cdot, \cdot)$. This form is nondegenerate on $\mathfrak{z}_{\chi}(0)$, hence we can find bases $\left\{a_{i}\right\}$ and $\left\{b_{i}\right\}$ of $\mathfrak{z}_{\chi}(0)$ such that $\left(a_{i}, b_{j}\right)=\delta_{i j}$. Set $\Theta_{\text {Cas }}:=\sum_{i} \Theta_{a_{i}} \Theta_{b_{i}}$, a central element of the associative subalgebra of $H$ generated by the Lie algebra $\Theta\left(\mathfrak{z}_{\chi}(0)\right)$. Obviously, we can regard $C$ as a central element of $H$.

By a well-known result of Joseph, outside type A the universal enveloping algebra $U(\mathfrak{g})$ contains a unique completely prime primitive ideal whose associated variety is $\overline{\mathcal{O}}_{\text {min }}$; see [14]. This ideal, often denoted $\mathcal{J}_{0}$, is known as the Joseph ideal of $U(\mathfrak{g})$.

We are finally in a position to formulate one of the main results of this article:

Theorem 1.1. The algebra $H$ is generated by the Casimir element $C$ and the subspaces $\Theta\left(\mathfrak{z}_{\chi}(i)\right)$ for $i=0,1$, subject to the following relations:

(i) $\left[\Theta_{x}, \Theta_{y}\right]=\Theta_{[x, y]}$ for all $x, y \in \mathfrak{z}_{\chi}(0)$;

(ii) $\left[\Theta_{x}, \Theta_{u}\right]=\Theta_{[x, u]}$ for all $x \in \mathfrak{z}_{\chi}(0)$ and $u \in \mathfrak{z}_{\chi}(1)$;

(iii) $C$ is central in $H$;

(iv) $\left[\Theta_{u}, \Theta_{v}\right]=\frac{1}{2}(f,[u, v])\left(C-\Theta_{\text {Cas }}-c_{0}\right)+\frac{1}{2} \sum_{i=1}^{2 s}\left(\Theta_{\left[u, z_{i}\right]^{\sharp}} \Theta_{\left[v, z_{i}^{*}\right]^{\sharp}}+\Theta_{\left[v, z_{i}^{*}\right]^{\sharp}} \Theta_{\left[u, z_{i}\right]^{\sharp}}\right)$ for all $u, v \in \mathfrak{z} \chi(1)$, where $c_{0}$ is a constant depending on $\mathfrak{g}$.

If $\mathfrak{g}$ is not of type $\mathrm{A}$ then $c_{0}$ is the eigenvalue of $C$ on the primitive quotient $U(\mathfrak{g}) / \mathcal{J}_{0}$. If $\mathfrak{g}$ is of type $\mathrm{A}_{n}, n \geq 2$, then $c_{0}=-n(n+1) / 4$. If $\mathfrak{g}$ is of type $\mathrm{A}_{1}$ then $H=\mathbb{k}[C]$.

We start proving this theorem in Section 2 where we show that (i) and (ii) hold in $H$ for a suitable choice of $\Theta: \mathfrak{z}_{\chi} \rightarrow H$. In Section 4 we determine all of the quadratic relation (iv) except the elusive constant $c_{0}$.

1.5. We first computed $c_{0}$ by brute force, but later it turned out that there was a much better way to do it, based on a certain refined version of Joseph's Preparation Theorem. 
This theorem, which we prove in Section 5 in our special case, enables us to link the primitive ideals of $H$ directly with primitive ideals of $U(\mathfrak{g})$.

Let $\Delta$ denote the automorphism of the polynomial algebra $\mathbb{k}[h]$ such that $\Delta(h)=$ $h+1$. Let $\langle\Delta\rangle$ stand for the cyclic subgroup of Aut $(\mathbb{k}[h])$ generated by $\Delta$. The skew group algebra $\mathbb{k}[h] *\langle\Delta\rangle$ has $\left\{h^{i} \Delta^{j} \mid i \in \mathbb{Z}_{+}, j \in \mathbb{Z}\right\}$ as a $\mathbb{k}$-basis and multiplication in $\mathbb{k}[h] *\langle\Delta\rangle$ has the property that $\Delta \cdot h=(h+1) \cdot \Delta$.

Let $\mathbf{A}_{e}$ denote the Weyl algebra with standard generators $z_{1}, \ldots, z_{s}, \partial_{1}, \ldots, \partial_{s}$, so that $\left[\partial_{i}, z_{j}\right]=\delta_{i j}$ for $1 \leq i, j \leq s$. Let $\mathcal{A}_{e}:=(\mathbb{k}[h] *\langle\Delta\rangle) \otimes \mathbf{A}_{e}$, a simple Noetherian algebra over $\mathbb{k}$, and identify $\mathbb{k}[h] *\langle\Delta\rangle$ and $\mathbf{A}_{e}$ with subalgebras of $\mathcal{A}_{e}$. Define an involution $\tau \in \operatorname{Aut}\left(\mathcal{A}_{e}\right)$ by setting

$$
\tau\left(z_{i}\right)=-z_{i}, \quad \tau\left(\partial_{i}\right)=-\partial_{i}, \quad \tau(h)=h, \quad \tau\left(\Delta^{k}\right)=(-1)^{k} \Delta^{k} \quad(1 \leq i \leq s, k \in \mathbb{Z}) .
$$

Then $\tau \otimes \sigma$ is an automorphism of order two of the associative algebra $\mathcal{A}_{e} \otimes H$.

Let $U(\mathfrak{g})_{f}$ denote the localisation of $U(\mathfrak{g})$ with respect to the Ore set $\left\{f^{i} \mid i \in \mathbb{Z}_{+}\right\}$. By mapping $U(\mathfrak{g})_{f}$ into the endomorphism algebra of the induced module $Q_{\chi}$ we are able to identify $U(\mathfrak{g})_{f}$ with a subalgebra of $\mathcal{A}_{e} \otimes H$. More precisely, we prove that

$$
U(\mathfrak{g})_{f}=\left(\mathcal{A}_{e} \otimes H\right)^{\tau \otimes \sigma}=\mathcal{A}_{e}^{\tau} \otimes H_{+} \oplus \mathcal{A}_{e}^{\tau} \Delta \otimes H_{-}
$$

where $H_{ \pm}=\{x \in H \mid \sigma(x)= \pm x\}$. As mentioned in 1.1 every two-sided ideal $I$ of $H$ is stable under the involution $\sigma \in \operatorname{Aut}(H)$. Hence $I=I_{+} \oplus I_{-}$where $I_{ \pm}=I \cap H_{ \pm}$. We identify $U(\mathfrak{g})$ with a subalgebra of $U(\mathfrak{g})_{f}$ and set

$$
\tilde{I}:=U(\mathfrak{g}) \cap\left(\mathcal{A}_{e}^{\tau} \otimes I_{+} \oplus \mathcal{A}_{e}^{\tau} \Delta \otimes I_{-}\right) .
$$

Then $\widetilde{I}$ is a two-sided ideal of $U(\mathfrak{g})$. By Corollary 5.1(vi), the centre of $H$ identifies canonically with $Z(\mathfrak{g})$, the centre of $U(\mathfrak{g})$. Let $X=\operatorname{Prim} U(\mathfrak{g})$ and let $X_{\text {inf }}$ be the set of all primitive ideals of infinite codimension in $\mathcal{X}$. Given a prime Noetherian ring $R$ we let $\operatorname{rk}(R)$ denote the Goldie rank of $R$.

Theorem 1.2. Take Prim $H$ with the Jacobson topology and take $X_{\mathrm{inf}}$ with the topology induced by the Jacobson topology of $X$. Then the following hold:

(i) The map $I \mapsto \widetilde{I}$ induces a homeomorphism $\varkappa$ : Prim $H \stackrel{\sim}{\rightarrow} X_{\text {inf. }}$.

(ii) For any $I \in \operatorname{Prim} H$ we have $\operatorname{Dim}(U(\mathfrak{g}) / \widetilde{I})=\operatorname{Dim}(H / I)+\operatorname{dim} \overline{\mathcal{O}}_{\text {min }}$.

(iii) If $I=\operatorname{Ann}_{H} V$ where $V$ is a finite-dimensional irreducible $H$-module, then $\widetilde{I}=$ $\operatorname{Ann}_{U(\mathfrak{g})}\left(Q_{\chi} \otimes_{H} V\right)$ and $\operatorname{rk}(U(\mathfrak{g}) / \widetilde{I})=\operatorname{dim} \operatorname{Wh}\left(Q_{\chi} \otimes_{H} V\right)=\operatorname{dim} V$.

(iv) For any $\mathcal{I} \in X$ with $\mathcal{V} \mathcal{A}(\mathcal{I})=\overline{\mathcal{O}}_{\text {min }}$ there is a finite-dimensional irreducible $H$ module $V$ such that $\mathcal{I}=\operatorname{Ann}_{U(\mathfrak{g})}\left(Q_{\chi} \otimes_{H} V\right)$.

(v) Let $V_{1}$ and $V_{2}$ be two finite-dimensional irreducible $H$-modules. Then $V_{1} \cong V_{2}$ as $H$-modules if and only if $\operatorname{Ann}_{U(\mathfrak{g})}\left(Q_{\chi} \otimes_{H} V_{1}\right)=\operatorname{Ann}_{U(\mathfrak{g})}\left(Q_{\chi} \otimes_{H} V_{2}\right)$.

(vi) A prime ideal $I$ of $H$ is primitive if and only if $I \cap Z(H)$ is a maximal ideal of $Z(H)$.

It follows from Theorem 1.2 that for any homomorphism $\eta: Z(\mathfrak{g}) \rightarrow \mathbb{k}$ the map $\varkappa$ induces a bijection between the isoclasses of finite-dimensional irreducible $H$-modules with central character $\eta$ and the primitive ideals $\mathcal{I} \in X$ such that $\mathcal{I} \cap Z(\mathfrak{g})=\operatorname{Ker} \eta$ and 
$\mathcal{V A}(\mathcal{I})=\overline{\mathcal{O}}_{\text {min }}($ recall that $Z(\mathfrak{g})=Z(H))$. This result indicates that for any nilpotent $\chi$ it should be possible to interpret the number of isoclasses of irreducible finite-dimensional $H_{\chi}$-modules with a fixed central character as the dimension of a cell representation of the integral Weyl group of the character. In type A this agrees with recent results of BrundanKleshchev.

Theorem 1.2(iii) relates the dimensions of irreducible finite-dimensional $H$-modules with Goldie-rank polynomials. We explore this in 6.4 to obtain dimension formulae for all irreducible finite-dimensional representations of $H$ for $\mathfrak{g}$ of type $\mathrm{C}_{n}$ and $\mathrm{G}_{2}$. It is quite possible that all Goldie-rank polynomials, properly scaled, will appear in dimension formulae for "nonrestricted Weyl modules" over Lie algebras of reductive groups in characteristic $p$ (we recall that in characteristic $p$ a truncated version of $H_{\chi}$ is Morita equivalent to the reduced enveloping algebra $U_{\chi}(\mathfrak{g})$; see [31, (2.3), (2.6)]).

Theorem 1.2(vi) says that $H$ satisfies the Dixmier-Mœglin-Rentschler equivalence. Again it is possible that this holds for any algebra $H_{\chi}$.

1.6. In the last section of this article we introduce and study highest weight modules for the algebra $H$. Let $\Phi_{e}=\{\alpha \in \Phi \mid \alpha(h)=0$ or 1$\}$, and put $\Phi_{e}^{ \pm}=\Phi_{e} \cap \Phi^{ \pm}$where $\Phi^{-}=-\Phi^{+}$. For $i=0,1$ put $\Phi_{e, i}^{ \pm}=\left\{\alpha \in \Phi_{e}^{ \pm} \mid \alpha(h)=i\right\}$. Note that $\mathfrak{z} \chi$ is spanned by $\mathfrak{h}_{e}:=\mathfrak{h} \cap \mathfrak{g}(0)^{\sharp}$, by root vectors $e_{\alpha}$ with $\alpha \in \Phi_{e}$, and by $e$. Let $h_{1}, \ldots, h_{l-1}$ be a basis of $\mathfrak{h}_{e}$, and let $\mathfrak{n}^{ \pm}(i)$ be the span of all $e_{\alpha}$ with $\alpha \in \Phi_{e, i}^{ \pm}$. Clearly, $\mathfrak{n}^{+}(0)$ and $\mathfrak{n}^{-}(0)$ are maximal nilpotent subalgebras of $\mathfrak{g}(0)^{\sharp}$. Let $\left\{x_{1}, \ldots, x_{t}\right\}$ and $\left\{y_{1}, \ldots, y_{t}\right\}$ be bases of $\mathfrak{n}^{+}(0)$ and $\mathfrak{n}^{-}(0)$ consisting of root vectors for $\mathfrak{h}$. For $1 \leq i \leq s$ let $\gamma_{i}$ (resp. $\left.\gamma_{i}^{*}\right)$ denote the root of $z_{i}$ (resp. $\left.z_{i}^{*}\right)$, and put $u_{i}=\left[e, z_{i}\right], u_{i}^{*}=\left[e, z_{i}^{*}\right]$. Then $\left\{u_{1}, \ldots, u_{s}, u_{1}^{*}, \ldots, u_{s}^{*}\right\}$ is a $\mathbb{k}$-basis of $\mathfrak{z} \chi(1)$.

In general, $H$ is unlikely to possess a triangular decomposition similar to that of $U(\mathfrak{g})$. Nevertheless, one can still define Verma modules and highest weight modules for $H$. Given $\lambda \in \mathfrak{h}_{e}^{*}$ and $c \in \mathbb{k}$ we denote by $J_{\lambda, c}$ the linear span in $H$ of all

$$
\prod_{i=1}^{t} \Theta_{y_{i}}^{l_{i}} \cdot \prod_{i=1}^{s} \Theta_{u_{i}}^{m_{i}} \cdot \prod_{i=1}^{\ell-1}\left(\Theta_{h_{i}}-\lambda\left(h_{i}\right)\right)^{n_{i}} \cdot(C-c)^{n_{\ell}} \cdot \prod_{i=1}^{s} \Theta_{u_{i}^{*}}^{r_{i}} \cdot \prod_{i=1}^{t} \Theta_{x_{i}}^{q_{i}}
$$

with $\sum_{i=1}^{\ell} n_{i}+\sum_{i=1}^{t} r_{i}+\sum_{i=1}^{s} q_{i}>0$. Using Theorem 1.1 we show in 7.1 that $J_{\lambda, c}$ is a left ideal of $H$. We call the $H$-module $Z_{H}(\lambda, c):=H / J_{\lambda, c}$ the Verma module of level $c$ corresponding to $\lambda$. By the above, $Z_{H}(\lambda, c)$ has a nice PBW basis. In 7.2 we show that $Z_{H}(\lambda, c)$ contains a unique maximal submodule which we denote $Z_{H}^{\max }(\lambda, c)$. Thus to every $(\lambda, c) \in \mathfrak{h}_{e}^{*} \times \mathbb{k}$ there corresponds an irreducible highest weight $H$-module $L_{H}(\lambda, c):=Z_{H}(\lambda, c) / Z_{H}^{\max }(\lambda, c)$. It is fairly easy to show that $L_{H}(\lambda, c) \cong L_{H}\left(\lambda^{\prime}, c^{\prime}\right)$ if and only if $(\lambda, c)=\left(\lambda^{\prime}, c^{\prime}\right)$ and that any irreducible finite-dimensional $H$-module is isomorphic to exactly one of $L_{H}(\lambda, c)$ with $\lambda$ satisfying a natural integrality condition.

To determine the composition multiplicities of the Verma modules $Z_{H}(\lambda, c)$ we link the latter with $\mathfrak{g}$-modules obtained by parabolic induction from Whittaker modules for $\mathfrak{s} \mathfrak{l}_{2}$. Let $\mathfrak{s}_{\beta}=\mathbb{k} e_{\beta} \oplus \mathbb{k} h_{\beta} \oplus \mathbb{k} f_{\beta}$ and put

$$
\mathfrak{p}_{\beta}:=\mathfrak{s}_{\beta}+\mathfrak{h}+\sum_{\alpha \in \Phi^{+}} \mathbb{k} e_{\alpha}, \quad \mathfrak{n}_{\beta}:=\sum_{\alpha \in \Phi^{+} \backslash\{\beta\}} \mathbb{k} e_{\alpha}, \quad \widetilde{\mathfrak{s}}_{\beta}:=\mathfrak{h}_{e} \oplus \mathfrak{s}_{\beta}
$$


Let $C_{\beta}=e f+f e+\frac{1}{2} h^{2}=2 e f+\frac{1}{2} h^{2}-h$, a central element of $U\left(\widetilde{s}_{\beta}\right)$. Given $\lambda \in \mathfrak{h}_{e}^{*}$ and $c \in \mathbb{k}$ we denote by $I_{\beta}(\lambda, c)$ the left ideal of $U\left(\mathfrak{p}_{\beta}\right)$ generated by $f-1, C_{\beta}-c$, all $h-\lambda(h)$ with $h \in \mathfrak{h}_{e}$, and all $e_{\gamma}$ with $\gamma \in \Phi^{+} \backslash\{\beta\}$. Let $Y(\lambda, c):=U\left(\mathfrak{p}_{\beta}\right) / I_{\beta}(\lambda, c)$, a $\mathfrak{p}_{\beta}$-module with the trivial action of $\mathfrak{n}_{\beta}$. Note that $Y(\lambda, c)$ is a Whittaker module for the Levi subalgebra $\widetilde{\mathfrak{s}}_{\beta}$. Now define

$$
M(\lambda, c):=U(\mathfrak{g}) \otimes_{U\left(\mathfrak{p}_{\beta}\right)} Y(\lambda, c) .
$$

Recall that each $z_{i}^{*}$ with $i \leq s$ is a root vector corresponding to $\gamma_{i}^{*}=-\beta-\gamma_{i} \in \Phi^{+}$. Let $\delta=\frac{1}{2}\left(\gamma_{1}^{*}+\cdots+\gamma_{s}^{*}\right)$ and $\rho=\frac{1}{2} \sum_{\alpha \in \Phi^{+}} \alpha$. Since the restriction of $(\cdot, \cdot)$ to $\mathfrak{h}_{e}$ is nondegenerate, for any $\eta \in \mathfrak{h}_{e}^{*}$ there is a unique $t_{\eta} \in \mathfrak{h}_{e}$ such that $\varphi=\left(t_{\eta}, \cdot\right)$. Hence $(\cdot, \cdot)$ induces a bilinear form on $\mathfrak{h}_{e}^{*}$ via $(\mu, v):=\left(t_{\mu}, t_{\nu}\right)$ for all $\mu, v \in \mathfrak{h}_{e}^{*}$. Given a linear function $\varphi \in \mathfrak{h}^{*}$ we denote by $\bar{\varphi}$ the restriction of $\varphi$ to $\mathfrak{h}_{e}$.

Theorem 1.3. Each $\mathfrak{g}$-module $M(\lambda, c)$ is an object of the category $\mathcal{C}_{\chi}$. Furthermore, $\mathrm{Wh}(M(\lambda, c)) \cong Z_{H}(\lambda+\bar{\delta}, c+(\lambda+2 \bar{\rho}, \lambda))$ as $H$-modules.

Combined with Skryabin's equivalence and the main results of Miličić-Soergel [28] and Backelin [1], Theorem 1.3 shows that the composition multiplicities of the Verma modules $Z_{H}(\lambda, c)$ can be computed with the help of certain parabolic Kazhdan-Lusztig polynomials. This confirms in the minimal nilpotent case the Kazhdan-Lusztig conjecture for finite $\mathcal{W}$-algebras formulated by de Vos and van Driel in [8]; see Remark 7.1 for more details.

Apart from its relevance to the theory of primitive ideals this work is a contribution to the rapidly growing theory of $\mathcal{W}$-algebras. Finite $\mathcal{W}$-algebras are attached to nilpotent elements of finite-dimensional simple Lie algebras via quantum Hamiltonian reduction. All finite $\mathcal{W}$-algebras of type A were recently described by J. Brundan and A. Kleshchev [5] who identified them with shifted truncated Yangians. It seems likely that their results can be extended to some nilpotent elements in Lie algebras of types B, C and D. Hidden Yangian symmetry of finite $\mathcal{W}$-algebras of type A was first discovered, in some special cases, by E. Ragoucy and P. Sorba [33].

Affine counterparts of finite $\mathcal{W}$-algebras have been studied even more intensively. It should be mentioned here that V. G. Kac and M. Wakimoto described minimal nilpotent superconformal algebras in the context of vertex operators and quantum reduction; see [20] and the references therein. It would be interesting to compare the algebras $H$ of this paper with quasiclassical limits of vertex algebras of Kac-Wakimoto.

\section{Structural features of the algebras $H_{\chi}$}

2.1. In this section we assume that $e$ is an arbitrary nilpotent element in $\mathfrak{g}$. Decompose $\mathfrak{g}$ into the weight spaces relative to ad $h$ giving a $\mathbb{Z}$-grading $\mathfrak{g}=\bigoplus_{i \in \mathbb{Z}} \mathfrak{g}(i)$. Let $\chi$ be as in 1.1 and denote by $\mathfrak{z} \chi$ the centraliser of $\chi$ in $\mathfrak{g}$. It is well-known that $\mathfrak{z} \chi=\mathfrak{c}_{\mathfrak{g}}(e)$ is a graded subalgebra of $\mathfrak{p}_{e}:=\bigoplus_{i \geq 0} \mathfrak{g}(i)$, that is, $\mathfrak{z}_{\chi}=\bigoplus_{i \geq 0} \mathfrak{z}_{\chi}(i)$ where $\mathfrak{z}_{\chi}(i)=\mathfrak{z} \chi \cap \mathfrak{g}(i)$. Choose a $\mathbb{k}$-basis $x_{1}, \ldots, x_{m}$ of the parabolic subalgebra $\mathfrak{p}_{e}$ with $x_{i} \in \mathfrak{g}\left(n_{i}\right)$ such that $x_{1}, \ldots, x_{r}$ $\operatorname{span} \mathfrak{z} \chi$. Let $\mathcal{O}=\mathcal{O}_{\chi}$ and let $d$ denote half of the dimension of $\mathcal{O}$. 
Define the skew-symmetric bilinear form $\langle\cdot, \cdot\rangle$ on the subspace $\mathfrak{g}(-1)$ by setting $\langle x, y\rangle=(e,[x, y])$ for all $x, y \in \mathfrak{g}(-1)$. As $\mathfrak{z}_{\chi} \subset \mathfrak{p}_{e}$, this form is nondegenerate. Choose a basis $z_{1}, \ldots, z_{s}, z_{s+1}, \ldots, z_{2 s}$ of $\mathfrak{g}(-1)$ such that

$$
\left\langle z_{i+s}, z_{j}\right\rangle=\delta_{i j}, \quad\left\langle z_{i}, z_{j}\right\rangle=\left\langle z_{i+s}, z_{j+s}\right\rangle=0 \quad(1 \leq i, j \leq r)
$$

and denote by $\mathfrak{g}(-1)^{0}$ the linear span of $z_{s+1}, \ldots, z_{2 s}$. Let $\mathfrak{m}_{\chi}=\mathfrak{g}(-1)^{0} \oplus \sum_{i \leq 2} \mathfrak{g}(i)$, a nilpotent subalgebra of $\mathfrak{g}$ of dimension $d$; see [31] for example. Since $\chi$ vanishes on the derived subalgebra of $\mathfrak{m}_{\chi}$ the ideal $N_{\chi}$ of $U\left(\mathfrak{m}_{\chi}\right)$ generated by all $x-\chi(x)$ with $x \in \mathfrak{m}_{\chi}$ has codimension one in the enveloping algebra $U\left(\mathfrak{m}_{\chi}\right)$. Let $\mathbb{k}_{\chi}=U\left(\mathfrak{m}_{\chi}\right) / N_{\chi}$, a onedimensional left $U\left(\mathfrak{m}_{\chi}\right)$-module, and let $1_{\chi}$ stand for the image of 1 in $\mathbb{k}_{\chi}$. We denote by $Q_{\chi}$ the induced $\mathfrak{g}$-module $U(\mathfrak{g}) \otimes_{U\left(\mathfrak{m}_{\chi}\right)} \mathbb{k}_{\chi}$ and set

$$
H_{\chi}:=\operatorname{End}_{\mathfrak{g}}\left(Q_{\chi}\right)^{\mathrm{op}}
$$

It is proved in [31] that the algebra $H_{\chi}$ is a filtered deformation of the graded coordinate ring $\mathbb{k}\left[\mathcal{S}_{e}\right]$.

In what follows we will rely on a different realisation of $H_{\chi}$ found by W. L. Gan and Ginzburg [10]. Let $\mathfrak{n}_{\chi}=\bigoplus_{i \leq-1} \mathfrak{g}(i)$ and $\mathfrak{n}_{\chi}^{\prime}=\bigoplus_{i \leq-2} \mathfrak{g}(i)$. Clearly, $\mathfrak{n}_{\chi}$ and $\mathfrak{n}_{\chi}^{\prime}$ are nilpotent subalgebras of $\mathfrak{g}$ and $\mathfrak{n}_{\chi}^{\prime}$ is an ideal of $\mathfrak{n}_{\chi}$. Since $\mathfrak{n}_{\chi}^{\prime} \subseteq \mathfrak{m}_{\chi}$, we may view $\mathbb{k}_{\chi}$ as an $\mathfrak{n}_{\chi}^{\prime}$-module. Let $\widehat{Q}_{\chi}=U(\mathfrak{g}) \otimes_{U\left(\mathfrak{n}_{\chi}^{\prime}\right)} \mathbb{k}_{\chi}$, an induced $\mathfrak{g}$-module and the quotient of $U(\mathfrak{g})$ by the left ideal $\mathcal{J}_{\chi}$ generated by all $x-\chi(x)$ with $x \in \mathfrak{n}_{\chi}^{\prime}$. The representation of $U(\mathfrak{g})$ in $\operatorname{End}(Q)$ will be denoted by $\widehat{\rho}_{\chi}$. Since $\chi$ vanishes on $\left[\mathfrak{n}_{\chi}, \mathfrak{n}_{\chi}^{\prime}\right] \subseteq \bigoplus_{i \leq-3} \mathfrak{g}(i)$, the left ideal $\mathcal{J}_{\chi}$ is stable under the adjoint action of $\mathfrak{n}_{\chi}$ on $U(\mathfrak{g})$. Therefore, ad $\mathfrak{n}_{\chi}$ acts on $\widehat{Q}_{\chi}$. The fixed point space $\widehat{Q}_{\chi}^{\mathrm{ad} \mathfrak{n}_{\chi}}$ carries a natural algebra structure given by $\left(x+\mathcal{J}_{\chi}\right)\left(y+\mathcal{J}_{\chi}\right)=x y+\mathcal{J}_{\chi}$ for all $x+\mathcal{J}_{\chi}, y+\mathcal{J}_{\chi} \in \widehat{Q}_{\chi}$; see [10, p. 244] for more details. We furnish $Q_{\chi}^{\text {ad } \mathfrak{m}_{\chi}}$ and $\widehat{Q}_{\chi}^{\text {ad } \mathfrak{n}_{\chi}^{\prime}}$ with algebra structures in a similar fashion. It is well known (and easily seen) that

$$
H_{\chi} \cong Q_{\chi}^{\mathrm{ad} \mathfrak{m}_{\chi}} \text { and } \operatorname{End}_{\mathfrak{g}}\left(\widehat{Q}_{\chi}\right)^{\mathrm{op}} \cong \widehat{Q}_{\chi}^{\mathrm{ad} \mathfrak{n}_{\chi}^{\prime}}
$$

as algebras. As $\mathfrak{n}_{\chi}^{\prime} \subseteq \mathfrak{m}_{\chi}$, there is a natural $\mathfrak{g}$-module epimorphism $\widehat{Q}_{\chi} \rightarrow Q_{\chi}$. As $\mathfrak{m}_{\chi} \subseteq \mathfrak{n}_{\chi}$, it induces an algebra map $\eta: \widehat{Q}_{\chi}^{\text {ad } \mathfrak{n}_{\chi}} \rightarrow H_{\chi}$. By [10, Theorem 4.1], $\eta$ is an isomorphism of algebras. Henceforth we will make no distinction between $H_{\chi}$ and $\widehat{Q}_{\chi}^{\text {ad } \mathfrak{n}_{\chi}}$ and view the latter as a subalgebra of $\operatorname{End}_{\mathfrak{g}}(\widehat{Q})^{\text {op }}$.

Given $(\mathbf{a}, \mathbf{b}) \in \mathbb{Z}_{+}^{m} \times \mathbb{Z}_{+}^{2 s}$ we set $x^{\mathbf{a}} z^{\mathbf{b}}:=x_{1}^{a_{1}} \cdots x_{m}^{a_{m}} z_{1}^{b_{1}} \cdots z_{2 s}^{b_{2 s}}$. By the PBW theorem, the monomials $x^{\mathbf{a}} z^{\mathbf{b}} \otimes 1_{\chi}$ with $(\mathbf{a}, \mathbf{b}) \in \mathbb{Z}_{+}^{m} \times \mathbb{Z}_{+}^{2 s}$ form a $\mathbb{k}$-basis of $\widehat{Q}_{\chi}$. For $k \in \mathbb{Z}_{+}$we denote by $\widehat{Q}_{\chi}^{k}$ the linear span of all $x^{\mathbf{a}} z^{\mathbf{b}} \otimes 1_{\chi}$ with

$$
|(\mathbf{a}, \mathbf{b})|_{e}:=\sum_{i=1}^{m} a_{i}\left(n_{i}+2\right)+\sum_{i=1}^{2 s} b_{i} \leq k .
$$

We let $H_{\chi}^{k}$ denote the subspace of $H_{\chi}$ consisting of all $h \in H_{\chi}$ with $h\left(1_{\chi}\right) \in \widehat{Q}_{\chi}^{k}$. By [31] and [10], the subspaces $\left\{H_{\chi}^{k} \mid k \in \mathbb{Z}_{+}\right\}$form an increasing filtration of the algebra 
$H_{\chi}$ and the corresponding graded algebra gr $H_{\chi}$ is isomorphic to a polynomial ring in $r$ variables with free homogeneous generators of degree $n_{1}+2, \ldots, n_{r}+2$. The elements $x$ in $\widehat{Q}_{\chi}^{k} \backslash \widehat{Q}_{\chi}^{k-1}$ and $H_{\chi}^{k} \backslash H_{\chi}^{k-1}$ are said to have Kazhdan degree $k$, written $\operatorname{deg}_{e}(x)=k$. It is immediate from [31, Theorem 4.6] that in our present realisation the algebra $H_{\chi}$ has a distinguished generating set $\Theta_{1}, \ldots, \Theta_{r}$ such that $\operatorname{gr} \Theta_{1}, \ldots, \operatorname{gr} \Theta_{r}$ generate gr $H_{\chi}$ and

$$
\Theta_{k}\left(1_{\chi}\right)=\left(x_{k}+\sum_{0<|(\mathbf{i}, \mathbf{j})|_{e} \leq n_{k}+2} \lambda_{\mathbf{i}, \mathbf{j}}^{k} x^{\mathbf{i}} z^{\mathbf{j}}\right) \otimes 1_{\chi}, \quad 1 \leq k \leq r,
$$

where $\lambda_{\mathbf{i}, \mathbf{j}}^{k} \in \mathbb{k}$ and $\lambda_{\mathbf{i}, \mathbf{j}}^{k}=0$ if either $|(\mathbf{i}, \mathbf{j})|_{e}=n_{k}+2$ and $|\mathbf{i}|+|\mathbf{j}|=1$, or $\mathbf{i} \neq \mathbf{0}, \mathbf{j}=\mathbf{0}$, and $i_{j}=0$ for $j>r$. The monomials $\Theta_{1}^{a_{1}} \ldots \Theta_{r}^{a_{r}}$ with $\left(a_{1}, \ldots, a_{r}\right) \in \mathbb{Z}_{+}^{r}$ form a PBW basis of $H_{\chi}$.

2.2. Given a subset $X$ of $\mathfrak{g}$ we denote by $Z_{G}(X)$ the closed subgroup of $G$ consisting of all $g \in G$ with $(\operatorname{Ad} g)(x)=x$ for all $x \in X$. Let $P_{e}$ denote the parabolic subgroup of $G$ with Lie $P_{e}=\mathfrak{p}_{e}$. There exists a 1-parameter subgroup $\lambda_{e}: \mathbb{k}^{\times} \rightarrow G$ optimal for the $G$-unstable vector $e$ and such that:

- $\left(\operatorname{Ad} \lambda_{e}(t)\right)_{\mid \mathfrak{g}(i)}=t^{i}$ id for all $t \in \mathbb{k}^{\times}$and $i \in \mathbb{Z}$;

- $Z_{G}(e) \subset P(e), R_{u}\left(Z_{G}(e)\right) \subset R_{u}\left(P_{e}\right), Z_{G}(e)=\left(Z_{G}(e) \cap Z_{G}\left(\lambda_{e}\right)\right) R_{u}\left(Z_{G}(e)\right)$;

- $C(e):=Z_{G}(e) \cap Z_{G}\left(\lambda_{e}\right)$ is a reductive group, and Lie $C(e)=\mathfrak{z}_{\chi}(0)$;

see [6, Chapter 5] and [32]. Let $\operatorname{Ad} C(e)$ denote the image of $C(e)$ in the adjoint group $\operatorname{Ad} G=(\text { Aut } \mathfrak{g})^{\circ}$. Put $\sigma=\operatorname{Ad} \lambda_{e}(-1)$, an element of order $\leq 2$ in Ad $G$. Clearly, $\sigma$ lies in the centre of $\operatorname{Ad} C(e)$ and $\sigma(x)=(-1)^{i} x$ for all $x \in \mathfrak{g}(i)$ and $i \in \mathbb{Z}$.

Lemma 2.1. The element $\sigma$ belongs to any maximal torus of $\operatorname{Ad} C(e)$.

Proof. Let $T_{0}$ be a maximal torus of $\operatorname{Ad} C(e), \widetilde{T}_{0}$ the inverse image of $T_{0}$ in $G$, and $L=Z_{G}\left(\widetilde{T}_{0}\right)$. Then $L$ is a Levi subgroup of $G$ and $e$ is a distinguished nilpotent element in $\mathfrak{l}=$ Lie $L$. The construction in [32] shows that all weights of $\operatorname{Ad} \lambda_{e}\left(\mathbb{k}^{\times}\right)$on $\mathfrak{l}$ are even. Then $\sigma$ acts trivially on $\mathfrak{l}$, yielding $\mathfrak{g}^{\sigma t} \supseteq \mathfrak{l}$ for all $t \in T_{0}$. As $\mathbb{k}$ is infinite, there is $t_{0} \in T_{0}$ such that $\mathfrak{g}^{\sigma t_{0}}=\mathfrak{l}$. Let $\mathcal{C}$ denote the conjugacy class of the image of $\sigma t_{0}$ in the component group $Z_{\operatorname{Ad} G}(e) / Z_{\operatorname{Ad} G}(e)^{\circ} \cong(\operatorname{Ad} C(e)) /(\operatorname{Ad} C(e))^{\circ}$. As $\operatorname{Ad} G$ is a group of adjoint type, the $G$-conjugacy class of the pair $(L, e)$ corresponds under Sommers' bijection to the $G$-conjugacy class of the pair $(e, \mathcal{C})$; see $[35,27,32]$. As $L$ is a Levi subgroup in $G$, the cited references also show that $\mathcal{C}=\{1\}$. But $T_{0} \subseteq(\operatorname{Ad} C(e))^{\circ}$ and $t_{0} \in T_{0}$. So we get $\sigma \in Z\left((\operatorname{Ad} C(e))^{\circ}\right)$. As ( $\left.\operatorname{Ad} C(e)\right)^{\circ}$ is a reductive group, the torus $T_{0}$ is self-centralising in $(\operatorname{Ad} C(e))^{\circ}$. Hence $\sigma \in T_{0}$, completing the proof.

We now fix a maximal torus $T_{e}$ in $\operatorname{Ad} C(e)$ and assume (without loss of generality) that all $z_{i}$ with $i \leq 2 s$ and $x_{j}$ with $j \leq m$ are weight vectors with respect to $T_{e}$. By Lemma 2.1. $\sigma \in T_{e}$. Note that $C(e)$ preserves both $\mathfrak{n}_{\chi}^{\prime}$ and Ker $\chi$. Since $C(e)$ acts on $U(\mathfrak{g})$ as algebra automorphisms, it preserves the left ideal $\mathcal{J}_{\chi}$ and thus acts on $\widehat{Q}_{\chi}$. This action is compatible with that of $\mathfrak{g}$, i.e.

$$
g \circ \widehat{\rho}_{\chi}(x) \circ g^{-1}=\widehat{\rho}_{\chi}((\operatorname{Ad} g)(x)) \quad(\forall g \in C(e), x \in \mathfrak{g}) .
$$


Since $C(e)$ preserves $\mathfrak{n}_{\chi}$ too, it acts on $H_{\chi}=\widehat{Q}_{\chi}^{\mathrm{ad} \mathfrak{n}_{\chi}}$ as algebra automorphisms. Since $g\left(1_{\chi}\right)=1_{\chi}$ for all $g \in C(e)$, the action of $C(e)$ on $\widehat{Q}_{\chi}$ and $H_{\chi}$ is filtration preserving, hence locally finite. Since $Z(G)$ acts trivially on $U(\mathfrak{g})$, there is a natural action of $\operatorname{Ad} C(e)$ on $\widehat{Q}_{\chi}$ and $H_{\chi}$. It should be mentioned that

$$
\sigma\left(x^{\mathbf{a}} z^{\mathbf{b}} \otimes 1_{\chi}\right)=(-1)^{|(\mathbf{a}, \mathbf{b})|_{e}} x^{\mathbf{a}} z^{\mathbf{b}} \otimes 1_{\chi}
$$

for all $(\mathbf{a}, \mathbf{b}) \in \mathbb{Z}_{+}^{m} \times \mathbb{Z}_{+}^{2 s}$.

Lemma 2.2. Each generator $\Theta_{k} \in H_{\chi}$ can be chosen to be a weight vector for $T_{e}$ of the same weight as $x_{k}$.

Proof. Let $\gamma_{k}$ denote the $T_{e}$-weight of $x_{k}$. If $\gamma_{k} \neq 0$, we assume without loss of generality that $\lambda_{\mathbf{0}, \mathbf{0}}^{k}=0$. Let $t \in T_{e}$ and $\Theta_{t}:=t\left(\Theta_{k}\right)-\gamma_{k}(t) \Theta_{k}$, an element in $H_{\chi}$. Since all $x^{\mathbf{i}} z^{\mathbf{j}} \otimes 1_{\chi} \in \widehat{Q}_{\chi}$ are weight vectors for $T_{e}$, we deduce from 2.1 .2 and 2.2 .1 that $\Theta_{t}\left(1_{\chi}\right)$ is a linear combination of $x^{\mathbf{a}} z^{\mathbf{b}} \otimes 1_{\chi}$ with either $\mathbf{b} \neq \mathbf{0}$ or $a_{j} \neq 0$ for some $j>r$. Then $\Theta_{t}=0$ for all $t \in T_{e}$, by [31, Lemma 4.5], and the result follows.

2.3. We now consider the linear map $\Theta: \mathfrak{z}_{\chi} \rightarrow H_{\chi}, x \mapsto \Theta_{x}$, such that $\Theta_{x_{i}}=\Theta_{i}$ for all $i$. Thanks to Lemma 2.2, $\Theta$ is an injective homomorphism of $T_{e}$-modules. Although $\Theta$ is not a Lie algebra homomorphism, in general, it follows from [31, Theorem 4.6(iv)] and 2.2.2 and Lemma 2.2 that

$$
\left[\Theta_{x_{i}}, \Theta_{x_{j}}\right] \equiv \Theta_{\left[x_{i}, x_{j}\right]}+q_{i j}\left(\Theta_{1}, \ldots, \Theta_{r}\right)\left(\bmod H_{\chi}^{n_{i}+n_{j}}\right)
$$

where $q_{i j}$ is a polynomial in $r$ variables with initial form of total degree $\geq 2$.

Remark 2.1. As $C(e)$ is a reductive group, each $C(e)$-module $H_{\chi}^{k}$ is completely reducible. From this it follows that there exists a unitriangular polynomial substitution

$$
F:\left(\Theta_{1}, \ldots, \Theta_{r}\right) \mapsto\left(F_{1}\left(\Theta_{1}, \ldots, \Theta_{r}\right), \ldots, F_{r}\left(\Theta_{1}, \ldots, \Theta_{r}\right)\right)
$$

which satisfies the following conditions:

- $\operatorname{deg}_{e} F\left(\Theta_{i}\right)=\operatorname{deg}_{e} \Theta_{i}=n_{i}+2$ for all $i \leq r$;

- the linear map $\Theta_{F}: \mathfrak{z} \chi \rightarrow H_{\chi}$ with $\Theta_{F}\left(x_{i}\right)=F\left(\Theta_{i}\right)$ for all $i$ is an injective homomorphism of $C(e)$-modules, and $\mathfrak{z}_{\chi} \cong \operatorname{gr} \Theta_{F}\left(\mathfrak{z}_{\chi}\right)$ as graded $C(e)$-modules;

- an analogue of 2.3 .1 holds for $F\left(\Theta_{1}\right), \ldots, F\left(\Theta_{r}\right)$, and the subspaces $\Theta_{F}\left(\mathfrak{z}_{\chi}\right)$ and gr $\Theta_{F}\left(\mathfrak{z}_{\chi}\right)$ generate the algebras $H_{\chi}$ and gr $H_{\chi}$, respectively.

Proposition 2.1. There exists an associative $\mathbb{k}[t]$-algebra $\mathcal{H}_{\chi}$ free as a module over $\mathbb{k}[t]$ and such that

$$
\mathcal{H}_{\chi} /(t-\lambda) \mathcal{H}_{\chi} \cong \begin{cases}H_{\chi} & \text { if } \lambda \neq 0, \\ U(\mathfrak{z} \chi) & \text { if } \lambda=0\end{cases}
$$

as $\mathbb{k}$-algebras. In other words, the enveloping algebra $U\left(\mathfrak{z}_{\chi}\right)$ is a contraction of $H_{\chi}$. 
Proof. Consider the algebra $H(R)=R \otimes H_{\chi}$ over the ring of Laurent polynomials $R=$ $k\left[t, t^{-1}\right]$ obtained from $H_{\chi}$ by extension of scalars, and identify $H_{\chi}$ with the subspace $\mathbb{k} \otimes H_{\chi}$ of $H(R)$. Define an invertible $R$-linear transformation $\pi$ on $H(R)$ by setting

$$
\pi\left(\Theta_{1}^{k_{1}} \cdots \Theta_{r}^{k_{r}}\right)=t^{n_{1} k_{1}+\cdots+n_{r} k_{r}} \Theta_{1}^{k_{1}} \cdots \Theta_{r}^{k_{r}} \quad \forall\left(k_{1}, \ldots, k_{r}\right) \in \mathbb{Z}_{+}^{r}
$$

and extending to $H(R)$ by $R$-linearity. We view $\pi$ as an isomorphism from $H(R)$ onto a new $R$-algebra $H(R, \pi)$ with underlying $R$-module $R \otimes H_{\chi}$ and with associative product given by $(x \cdot y)_{\pi}:=\pi^{-1}(\pi(x) \cdot \pi(y))$ for all $x, y \in R \otimes H_{\chi}$. We denote by $\mathcal{H}_{\chi}$ the free $\mathbb{k}[t]$-submodule of $H(R, \pi)$ generated by $\Theta_{1}^{a_{1}} \ldots \Theta_{r}^{a_{r}}$ with $\left(a_{1}, \ldots, a_{r}\right) \in \mathbb{Z}_{+}^{r}$. It follows from 2.1.1 and 2.1.2 that

$$
\operatorname{deg}_{e}\left(\Theta_{1}^{k_{1}} \ldots \Theta_{r}^{k_{r}}\right)=\sum_{i=1}^{r} n_{i} k_{i}+2 \sum_{i=1}^{r} k_{i} .
$$

In view of 2.3.1 this yields

$$
\left(\Theta_{i} \cdot \Theta_{j}-\Theta_{j} \cdot \Theta_{i}\right)_{\pi}=\pi^{-1}\left(t^{n_{i}+n_{j}}\left[\Theta_{i}, \Theta_{j}\right]\right) \equiv \Theta_{\left[x_{i}, x_{j}\right]}\left(\bmod t \mathcal{H}_{\chi}\right)
$$

(since the initial form of $q_{i j}$ has total degree $\geq 2$ and $\operatorname{deg}_{e} q_{i j}\left(\Theta_{1}, \ldots, \Theta_{r}\right)=n_{i}+n_{j}+2$ if $q_{i j} \neq 0$ ). Using induction on the Kazhdan degree of $\Theta_{1}^{k_{1}} \cdots \Theta_{r}^{k_{r}}$ and the commutativity of gr $H_{\chi}$ we now deduce that $\left(\Theta_{i} \cdot \mathcal{H}_{\chi}\right)_{\pi} \subseteq \mathcal{H}_{\chi}$ for all $i$. So $\mathcal{H}_{\chi}$ is a $\mathbb{k}[t]$-subalgebra of $H(R, \pi)$.

If $\lambda \neq 0$ then the homomorphism $\mathbb{k}[t] \rightarrow k$ taking $t$ to $\lambda$ extends to a homomorphism $R \rightarrow k$. The isomorphism $\pi^{-1}$ injects $(t-\lambda) H(R, \pi)$ onto $(t-\lambda) H(R)$. Because $\mathcal{H}_{\chi} \cap(t-\lambda) H(R, \pi)=(t-\lambda) \mathcal{H}_{\chi}$ and $H_{\chi} \cap(t-\lambda) H(R)=0$, we have

$$
\mathcal{H}_{\chi} /(t-\lambda) \mathcal{H}_{\chi} \cong H(R, \pi) /(t-\lambda) H(R, \pi) \cong H(R) /(t-\lambda) H(R) \cong H_{\chi},
$$

by the theorem on isomorphism. Now put $\overline{\mathcal{H}}_{\chi}:=\mathcal{H}_{\chi} / t \mathcal{H}_{\chi}$ and identify the generators $\Theta_{i}=\Theta_{x_{i}}$ of $\mathcal{H}_{\chi}$ with their images in $\overline{\mathcal{H}}_{\chi}$. It is immediate from our earlier remarks that these images satisfy the relations $\left[\Theta_{x_{i}}, \Theta_{x_{j}}\right]=\Theta_{\left[x_{i}, x_{j}\right]}$ for all $i, j$. By the universality property of the enveloping algebra $U\left(\mathfrak{z}_{\chi}\right)$, there exists an algebra homomorphism $\phi: U\left(\mathfrak{z}_{\chi}\right) \rightarrow \overline{\mathcal{H}}_{\chi}$ with $\phi\left(x_{i}\right)=\Theta_{i}$ for all $i$. Since $\mathcal{H}_{\chi}$ is a free $\mathbb{k}[t]$-module, the monomials $\Theta_{1}^{a_{1}} \ldots \Theta_{r}^{a_{r}}$ with $\left(a_{1}, \ldots, a_{r}\right) \in \mathbb{Z}_{+}^{r}$ are linearly independent in $\overline{\mathcal{H}}_{\chi}$. As a consequence, $\phi$ is an isomorphism.

2.4. Let $\mathbf{A}_{e}$ denote the associative algebra over $\mathbb{k}$ generated by $z_{1}, \ldots, z_{s}, z_{s+1}, \ldots, z_{2 s}$ subject to the relations $\left[z_{i+s}, z_{j}\right]=\delta_{i j}$ and $\left[z_{i}, z_{j}\right]=\left[z_{i+s}, z_{j+s}\right]=0$ where $1 \leq i, j \leq s$. Clearly, $\mathbf{A}_{e} \cong \mathbf{A}_{s}(\mathbb{k})$, the $s$ th Weyl algebra over $\mathbb{k}$. If $s=0$ then $\mathbf{A}_{e}=\mathbb{k}$.

Let $i \mapsto i^{*}$ denote the involution on the set of indices $\{1, \ldots, s, s+1, \ldots, 2 s\}$ such that $i^{*}=i+s$ for $i \leq s$ and $i^{*}=i-s$ for $i>s$. For $1 \leq i \leq 2 s$ define $z_{i}^{*}:=(-1)^{p(i)} z_{i}{ }^{*}$ where

$$
p(i)= \begin{cases}0 & \text { if } i \leq s \\ 1 & \text { if } i>s\end{cases}
$$


Note that $z_{i}=(-1)^{p\left(i^{*}\right)} z_{i^{*}}^{*}$ for $1 \leq i \leq 2 s$ and $z_{i}^{*}=z_{i+s}, z_{i+s}^{*}=-z_{i}$ for $1 \leq i \leq s$. It is worth remarking that the following relation holds in $U(\mathfrak{g})$ :

$$
\sum_{i=1}^{2 s} z_{i} z_{i}^{*}=-\sum_{i=1}^{2 s} z_{i}^{*} z_{i} \equiv s\left(\bmod \mathcal{J}_{\chi}\right) .
$$

As the form $\langle\cdot, \cdot\rangle$ is $\mathfrak{z} \chi(0)$-invariant and $\left\langle z_{i}^{*}, z_{j}\right\rangle=\delta_{i j}$ for $1 \leq i, j \leq 2 s$, for all $x \in \mathfrak{z} \chi(0)$ we have

$$
\left[x, z_{k}^{*}\right]=\sum_{i=1}^{2 s}\left\langle z_{k}^{*},\left[x, z_{i}^{*}\right]\right\rangle z_{i}=-\sum_{i=1}^{2 s}\left\langle\left[x, z_{i}^{*}\right], z_{k}^{*}\right\rangle z_{i} .
$$

Each $h \in H_{\chi}$ is determined by its effect on the canonical generator $1_{\chi} \in \widehat{Q}_{\chi}$. Since the vector $h\left(1_{\chi}\right)$ can be uniquely expressed as $h\left(1_{\chi}\right)=\left(\sum_{\mathbf{i} \in \mathbb{Z}_{+}^{2 s}} u_{\mathbf{i}} \cdot z^{\mathbf{i}}\right) \otimes 1_{\chi}$ with $u_{\mathbf{i}} \in$ $U\left(\mathfrak{p}_{e}\right)$, one obtains a natural linear injection

$$
\tilde{\mu}: H_{\chi} \rightarrow U\left(\mathfrak{p}_{e}\right) \otimes \mathbf{A}_{e}^{\mathrm{op}}, \quad \tilde{\mu}(h)=\sum_{\mathbf{i} \in \mathbb{Z}_{+}^{2 s}} u_{\mathbf{i}} \otimes z^{\mathbf{i}} .
$$

As the form $\langle\cdot, \cdot\rangle$ is $C(e)$-invariant, the group $C(e)$ acts on $\mathbf{A}_{e}^{\mathrm{op}}$ as automorphisms. As $C(e)$ also acts on $U\left(\mathfrak{p}_{e}\right)$, it acts as automorphisms on the algebra $U\left(\mathfrak{p}_{e}\right) \otimes \mathbf{A}_{e}^{\text {op }}$, via $g(u \otimes a)=g(u) \otimes g(a)$ with the obvious choices of $g, u, a$.

Proposition 2.2. The map $\tilde{\mu}: H_{\chi} \hookrightarrow U\left(\mathfrak{p}_{e}\right) \otimes \mathbf{A}_{e}^{\mathrm{op}}$ is a $C($ e)-equivariant algebra homomorphism.

Proof. Let $z$ denote the linear span of all $z^{\mathbf{i}} \otimes 1_{\chi}$ with $\mathbf{i} \in \mathbb{Z}_{+}^{2 s}$. We identify $z$ with the space of the left regular representation of $\mathbf{A}_{e}$ via $z^{\mathbf{i}} \otimes 1_{\chi} \mapsto z^{\mathbf{i}}$. Now $\widehat{\rho}_{\chi}$ induces a representation of $U\left(\mathfrak{n}_{\chi}\right)$ in $\operatorname{End}(Z)$, say $\psi_{0}$. Since $\mathfrak{g}(-1) \subset \mathfrak{n}_{\chi}$ and $\mathfrak{g}(i) \subset \operatorname{Ker} \chi$ for all $i \leq-3$, the definition of $\widehat{Q}_{\chi}^{\text {ad } \mathfrak{n}_{\chi}}$ and induction on $k$ show that

$$
\widehat{\rho}_{\chi}\left(z_{1} \cdots z_{k}\right)\left(h\left(1_{\chi}\right)\right)=\sum_{\mathbf{i} \in \mathbb{Z}_{+}^{2 s}} u_{\mathbf{i}} \cdot \widehat{\rho}_{\chi}\left(z^{\mathbf{i}} \cdot z_{1} \cdots z_{k}\right)\left(1_{\chi}\right)
$$

for all $z_{1}, \ldots, z_{k} \in \mathfrak{g}(-1)$. Now let $h^{\prime}$ be another element in $H_{\chi}$ and suppose that $h^{\prime}\left(1_{\chi}\right)=\left(\sum_{\mathbf{i} \in \mathbb{Z}_{+}^{2 s}} u_{\mathbf{i}}^{\prime} \cdot z^{\mathbf{i}}\right) \otimes 1_{\chi}$ where $u_{\mathbf{i}}^{\prime} \in U\left(\mathfrak{p}_{e}\right)$. Then

$$
\begin{aligned}
\left(h \cdot h^{\prime}\right)\left(1_{\chi}\right) & =h^{\prime}\left(h\left(1_{\chi}\right)\right)=\sum_{\mathbf{i}} \widehat{\rho}_{\chi}\left(u_{\mathbf{i}}\right) \cdot \widehat{\rho}_{\chi}\left(z^{\mathbf{i}}\right)\left(h^{\prime}\left(1_{\chi}\right)\right) \\
& =\sum_{\mathbf{i}} \sum_{\mathbf{j}} u_{\mathbf{i}} \cdot u_{\mathbf{j}}^{\prime} \cdot \widehat{\rho}_{\chi}\left(z^{\mathbf{j}} \cdot z^{\mathbf{i}}\right)\left(1_{\chi}\right) .
\end{aligned}
$$

It remains to note that the map $z^{\mathbf{i}} \otimes 1_{\chi} \mapsto z^{\mathbf{i}}$ mentioned above identifies $\psi_{0}\left(U\left(\mathfrak{n}_{\chi}\right)\right)$ with the image of $\mathbf{A}_{e}$ in its left regular representation. The $C(e)$-equivariance of $\tilde{\mu}$ is immediate from the definitions. 
Remark 2.2. Composing $\tilde{\mu}$ with the natural projection $U\left(\mathfrak{p}_{e}\right) \otimes \mathbf{A}_{e}^{\mathrm{op}} \rightarrow U(\mathfrak{g}(0)) \otimes \mathbf{A}_{e}^{\mathrm{op}}$ one obtains an algebra homomorphism

$$
\mu: H_{\chi} \rightarrow U(\mathfrak{g}(0)) \otimes \mathbf{A}_{e}^{\mathrm{op}}
$$

which will be referred to as the Miura map. In the special case where $e$ is even this map has already appeared in $[31,(7.1)]$ (note that for $e$ even we have $\mathbf{A}_{e}^{\mathrm{op}}=\mathbb{k}$ ). It can be proved that the map $\mu$ is always injective (this will not be required in the present article).

The adjoint action of $\mathfrak{z}_{\chi}(0)$ on $\mathfrak{g}$ induces Lie algebra maps $\mathfrak{z}_{\chi}(0) \rightarrow \operatorname{Der}\left(\mathbf{A}_{e}^{\mathrm{op}}\right)$, $\mathfrak{z}_{\chi}(0) \rightarrow \operatorname{Der}\left(U\left(\mathfrak{p}_{e}\right) \otimes \mathbf{A}_{e}^{\mathrm{op}}\right)$ and $\mathfrak{z}_{\chi}(0) \rightarrow \operatorname{Der}\left(H_{\chi}\right)$ (of course, the same maps can be obtained by differentiating the respective actions of $C(e)$ on $\mathbf{A}_{e}^{\mathrm{op}}, U\left(\mathfrak{p}_{e}\right) \otimes \mathbf{A}_{e}^{\mathrm{op}}$ and $\left.H_{\chi}\right)$. By abuse of notation, the image of $x \in \mathfrak{z}_{\chi}(0)$ under each of these maps will be denoted by ad $x$.

2.5. In what follows we will need explicit formulae for the generators $\Theta_{k}$ of small Kazhdan degree. The reader will observe strong similarity between our formulae and the expressions for conserved fields of low conformal weight found by Kac and Wakimoto [20] in the context of vertex algebras and quantum reduction.

Lemma 2.3. If $v \in \mathfrak{z}_{\chi}(0)$ then it can be assumed that

$$
\Theta_{v}\left(1_{\chi}\right)=\left(v+\frac{1}{2} \sum_{i=1}^{2 s} z_{i}\left[v, z_{i}^{*}\right]\right) \otimes 1_{\chi}=\left(v+\frac{1}{2} \sum_{i=1}^{2 s}\left[v, z_{i}^{*}\right] z_{i}\right) \otimes 1_{\chi} .
$$

Proof. It follows from 2.1.2 and 2.2.2 that there exist a scalar $\beta$ and a symmetric matrix $A=\left(\alpha_{i j}\right)$ of order $2 s$ such that

$$
\Theta_{v}\left(1_{\chi}\right)=\left(v+\frac{1}{2} \sum_{i, j=1}^{2 s} \alpha_{i j} z_{i} z_{j}+\beta\right) \otimes 1_{\chi} .
$$

Since $A$ is symmetric and $\left[z_{k}^{*}, v+\frac{1}{2} \sum_{i, j=1}^{2 s} \alpha_{i j} z_{i} z_{j}+\beta\right] \in \mathcal{J}_{\chi}$ for all $k$, it must be that $\left[v, z_{k}^{*}\right]=\sum_{j=1}^{2 s} \alpha_{k j} z_{j}$. Therefore, after a proper adjustment of $\beta$ we get

$$
\Theta_{v}\left(1_{\chi}\right)=\left(v+\frac{1}{2} \sum_{i=1}^{2 s} z_{i}\left[v, z_{i}^{*}\right]\right) \otimes 1_{\chi} .
$$

Since $\mathcal{J}_{\chi}$ is $(\operatorname{ad} v)$-stable, 2.4.1 yields $\sum_{i=1}^{2 s} z_{i}\left[v, z_{i}^{*}\right] \equiv \sum_{i=1}^{2 s}\left[v, z_{i}^{*}\right] z_{i}\left(\bmod \mathcal{J}_{\chi}\right)$. This completes the proof.

From now on we always assume that the generators $\Theta_{v}$ with $v \in \mathfrak{z}_{x}(0)$ are chosen in accordance with Lemma 2.3 . This has the following advantage: 
Lemma 2.4. The restriction of $\Theta$ to $\mathfrak{z}_{\chi}(0)$ is a Lie algebra homomorphism, i.e.

$$
\left[\Theta_{u}, \Theta_{v}\right]=\Theta_{[u, v]} \quad\left(\forall u, v \in \mathfrak{z}_{\chi}(0)\right) .
$$

Moreover, the Lie algebra homomorphism ad $\circ \Theta: \mathfrak{z} \chi(0) \rightarrow \operatorname{Der}\left(H_{\chi}\right)$ coincides with the differential of the rational action $C(e) \rightarrow \operatorname{Aut}\left(H_{\chi}\right)$.

Proof. We are going to use the injective homomorphism $\tilde{\mu}$ from 2.4 Let $x \in \mathfrak{z}_{\chi}(0)$. Computing in $\mathbf{A}_{e}^{\mathrm{op}}$ and applying 2.4 .2 we get

$$
\begin{aligned}
{\left[\frac{1}{2} \sum_{i=1}^{2 s}\left[x, z_{i}^{*}\right] z_{i}, z\right] } & =-\frac{1}{2} \sum_{i=1}^{2 s}\left(\left\langle\left[x, z_{i}^{*}\right], z\right\rangle z_{i}+\left\langle z_{i}, z\right\rangle\left[x, z_{i}^{*}\right]\right) \\
& =\frac{1}{2}([x, z]+[x, z])=[x, z]
\end{aligned}
$$

for all $z \in \mathfrak{g}(-1)$. Hence $\operatorname{ad} x=\operatorname{ad}\left(\frac{1}{2} \sum_{i=1}^{2 s}\left[x, z_{i}^{*}\right] z_{i}\right)$ as derivations of $\mathbf{A}_{e}^{\text {op }}$. Then

$\left[\tilde{\mu}\left(\Theta_{x}\right), \tilde{\mu}(h)\right]=\left[\tilde{\mu}\left(\Theta_{x}\right), \sum u_{\mathbf{i}} \otimes z^{\mathbf{i}}\right]=\sum\left(\left[x, u_{\mathbf{i}}\right] \otimes z^{\mathbf{i}}+u_{\mathbf{i}} \otimes\left[x, z^{\mathbf{i}}\right]\right)=\tilde{\mu}((\operatorname{ad} x)(h))$ for all $h \in H_{\chi}$. As $\tilde{\mu}$ is injective, it must be that $\left[\Theta_{x}, h\right]=(\operatorname{ad} x)(h)$, i.e. the adjoint action of $\Theta\left(\mathfrak{z}_{\chi}(0)\right)$ coincides with the differential of the action of $C(e)$ on $H_{\chi}$. Also,

$$
\begin{aligned}
\sum_{i=1}^{2 s}\left[v, z_{i}^{*}\right]\left[u, z_{i}\right] & \stackrel{2.4 .2}{=}-\sum_{i, j=1}^{2 s}\left\langle z_{j}^{*},\left[u, z_{i}^{*}\right]\right\rangle\left[v, z_{i}\right] z_{j} \\
& \underline{\underline{2.4 .2}}-\sum_{i, j=1}^{2 s}\left\langle z_{i}^{*},\left[u, z_{j}^{*}\right]\right\rangle\left[v, z_{i}\right] z_{j}=-\sum_{j=1}^{2 s}\left[v,\left[u, z_{j}^{*}\right]\right] z_{j}
\end{aligned}
$$

as elements in $\mathbf{A}_{e}^{\text {op }}$, for all $u, v \in \mathfrak{z}_{\chi}(0)$. It follows that

$$
\begin{aligned}
{\left[\tilde{\mu}\left(\Theta_{u}\right), \tilde{\mu}\left(\Theta_{v}\right)\right] } & \stackrel{2.5 .1}{=}[u, v] \otimes 1+\frac{1}{2} \otimes \sum_{i=1}^{2 s}\left(\left[u,\left[v, z_{i}^{*}\right]\right] z_{i}+\left[v, z_{i}^{*}\right]\left[u, z_{i}\right]\right) \\
& \stackrel{\underline{2.5 .2}}{=}[u, v] \otimes 1+\frac{1}{2} \otimes \sum_{i=1}^{2 s}\left[u,\left[v, z_{i}^{*}\right]\right] z_{i}-\frac{1}{2} \otimes \sum_{i=1}^{2 s}\left[v,\left[u, z_{i}^{*}\right]\right] z_{i} \\
& =[u, v] \otimes 1+\frac{1}{2} \otimes \sum_{i=1}^{2 s}\left[[u, v], z_{i}^{*}\right] z_{i}=\tilde{\mu}\left(\Theta_{[u, v]}\right) .
\end{aligned}
$$

But then $\left[\Theta_{u}, \Theta_{v}\right]=\Theta_{[u, v]}$ for all $u, v \in \mathfrak{z}_{\chi}(0)$, as stated.

Corollary 2.1. Any two-sided ideal of $H_{\chi}$ is $\sigma$-stable. 
Proof. Let $I$ be a two-sided ideal of $H_{\chi}$. Clearly, $I$ is invariant under the adjoint action of $\Theta\left(\mathfrak{z}_{\chi}(0)\right)$. By Lemma 2.4, $I$ is then stable under the differential of the $C(e)$-action on $H_{\chi}$. Since $C(e)^{\circ}$ is a connected reductive group and the action of $C(e)$ on $H_{\chi}$ is filtration preserving, Weyl's theorem on complete reducibility shows that all subspaces $I \cap H_{\chi}^{k}$ are $C(e)^{\circ}$-stable. Since $Z(G) \subseteq C(e)$ acts trivially on $H_{\chi}$ and $(\operatorname{Ad} C(e))^{\circ}$ coincides with the image of $C(e)^{\circ}$ in $\operatorname{Ad} C(e) \cong C(e) / Z(G)$, Lemma 2.1 shows that $I$ is $\sigma$-stable, as claimed.

Given $n$ elements $x_{1}, x_{2}, x_{3}, \ldots, x_{n}$ in a Lie algebra we denote by $\left[x_{1} x_{2} x_{3} \ldots x_{n}\right]$ the commutator $\left[\ldots\left[\left[x_{1}, x_{2}\right], x_{3}\right], \ldots, x_{n}\right]$.

Lemma 2.5. If $v \in \mathfrak{z}_{\chi}(1)$ then the generator $\Theta_{v} \in H_{\chi}$ has the following property:

$$
\Theta_{v}\left(1_{\chi}\right)=\left(v+\sum_{i=1}^{2 s}\left[v, z_{i}^{*}\right] z_{i}+\frac{1}{3} \sum_{i, j=1}^{2 s}\left[v z_{i}^{*} z_{j}^{*}\right] z_{j} z_{i}+z_{v}\right) \otimes 1_{\chi}
$$

where $z_{v}=\frac{1}{3} \sum_{i=1}^{2 s}\left(\sum_{k=1}^{2 s}\left\langle z_{k},\left[v,\left[z_{k}^{*}, z_{i}^{*}\right]\right]\right\rangle\right) z_{i}$. Moreover,

$$
\left[\Theta_{u}, \Theta_{v}\right]=\Theta_{[u, v]} \quad\left(\forall u \in \mathfrak{z}_{\chi}(0)\right) .
$$

Proof. Let $h_{v}=\sum_{i}\left[v, z_{i}^{*}\right] z_{i}+\frac{1}{3} \sum_{i, j}\left[v z_{i}^{*} z_{j}^{*}\right] z_{j} z_{i}+z_{v}$, an element in $U(\mathfrak{g})$. By anticommutativity and the Jacobi identity, we have

$$
\left[z_{k}^{*},\left[v z_{i}^{*} z_{j}^{*}\right]\right]=\left[z_{j}^{*},\left[v z_{i}^{*} z_{k}^{*}\right]\right]+\left[v\left[z_{k}^{*} z_{j}^{*}\right] z_{i}^{*}\right]+\left[v,\left[z_{i}^{*}\left[z_{k}^{*}, z_{j}^{*}\right]\right]\right] .
$$

Since $(e,[v, x])=0$ for all $x \in \mathfrak{g}$, this yields

$$
\left\langle z_{k}^{*},\left[v z_{i}^{*} z_{j}^{*}\right]\right\rangle=\left\langle z_{j}^{*},\left[v z_{i}^{*} z_{k}^{*}\right]\right\rangle-\left\langle z_{i}^{*},\left[v z_{k}^{*} z_{j}^{*}\right]\right\rangle+\left\langle z_{i}^{*},\left[v z_{j}^{*} z_{k}^{*}\right]\right\rangle
$$

where $1 \leq i, j, k \leq 2 s$. Computing in $U(\mathfrak{g})$ modulo $\mathcal{J}_{\chi}$ we now get

$$
\begin{aligned}
{\left[z_{k}^{*}, \sum_{i, j}\left[v z_{i}^{*} z_{j}^{*}\right] z_{j} z_{i}\right] \equiv } & \sum_{i j}\left\langle z_{k}^{*},\left[v z_{i}^{*} z_{j}^{*}\right]\right\rangle z_{j} z_{i}+\sum_{i}\left[v z_{i}^{*} z_{k}^{*}\right] z_{i}+\sum_{i}\left[v z_{k}^{*} z_{i}^{*}\right] z_{i} \\
= & \sum_{i j}\left(\left\langle z_{j}^{*},\left[v z_{i}^{*} z_{k}^{*}\right]\right\rangle-\left\langle z_{i}^{*},\left[v z_{k}^{*} z_{j}^{*}\right]\right\rangle+\left\langle z_{i}^{*},\left[v z_{j}^{*} z_{k}^{*}\right]\right\rangle\right) z_{j} z_{i} \\
& +\sum_{i}\left(\left[v z_{i}^{*} z_{k}^{*}\right] z_{i}+\left[v z_{k}^{*} z_{i}^{*}\right] z_{i}\right) \\
\equiv & \sum_{i}\left(\left[v z_{i}^{*} z_{k}^{*}\right] z_{i}-z_{i}\left[v z_{k}^{*} z_{i}^{*}\right]+z_{i}\left[v z_{i}^{*} z_{k}^{*}\right]\right) \\
\equiv & 3 \sum_{i}\left(\left[v z_{i}^{*} z_{k}^{*}\right] z_{i}-\frac{1}{3}\left\langle z_{i},\left[v z_{k}^{*} z_{i}^{*}\right]\right\rangle+\frac{1}{3}\left\langle z_{i},\left[v z_{i}^{*} z_{k}^{*}\right]\right\rangle\right) \\
\equiv & 3\left(\sum_{i}\left(\left[v z_{i}^{*} z_{k}^{*}\right] z_{i}\right)-3\left[z_{k}^{*}, z_{v}\right] .\right.
\end{aligned}
$$


As a consequence,

$$
\begin{aligned}
{\left[z_{k}^{*}, h_{v}\right] \equiv } & {\left[z_{k}^{*}, v\right]+\sum_{i}\left[z_{k}^{*},\left[v, z_{i}^{*}\right]\right] z_{i}+\left[v, z_{k}^{*}\right]+\left[z_{k}^{*}, z_{v}\right] } \\
& +\sum_{i}\left[v z_{i}^{*} z_{k}^{*}\right] z_{i}-\left[z_{k}^{*}, z_{v}\right] \equiv 0\left(\bmod \mathcal{J}_{\chi}\right)
\end{aligned}
$$

for all $k$. It is easy to see that $\left[z, h_{v}\right] \equiv 0\left(\bmod \mathcal{J}_{\chi}\right)$ for all $z \in \mathfrak{n}_{\chi}^{\prime}$. By Lemma 2.2 $\Theta_{v}$ is a $(-1)$-eigenvector for $\sigma$. In conjunction with 2.1.2], [31, Lemma 4.5], and the computation above this shows that $\Theta_{v}\left(1_{\chi}\right)=h_{v} \otimes 1_{\chi}$.

Now let $u$ be any element in $\mathfrak{z}_{\chi}(0)$ and put $\Theta^{\prime}:=\left[\Theta_{u}, \Theta_{v}\right]-\Theta_{[u, v]}$. It is immediate from 2.3.1 that $\Theta^{\prime}$ is a polynomial in $\Theta_{x_{i}}$ with $x_{i} \in \mathfrak{z}_{\chi}(0)$. Since $\sigma\left(\Theta^{\prime}\right)=-\Theta^{\prime}$ by Lemma 2.2 this polynomial must be zero. So $\left[\Theta_{u}, \Theta_{v}\right]=\Theta_{[u, v]}$ necessarily holds, completing the proof.

\section{Associated varieties and Gelfand-Kirillov dimension}

3.1. At present very little is known about finite-dimensional representations of the algebras $H_{\chi}$. In view of Proposition 2.1 this can be partly explained by the lack of detailed information on the structure of the centraliser $\mathfrak{c}_{\mathfrak{g}}(e)$. Besides, if $e$ is not even then there is no obvious reason for $H_{\chi}=H_{\chi_{e}}$ to possess such representations. On the other hand, the evidence collected so far suggests that each algebra $H_{\chi}$ has infinitely many isoclasses of finite-dimensional irreducible representations and dimension formulae for those have roughly the same format as the Weyl dimension formula for $H_{0}=U(\mathfrak{g})$; see 6.4 It is therefore natural to ask:

Question 3.1. Is it true that for any nonzero $h \in H_{\chi}$ there exists a finite-dimensional irreducible representation $\rho$ of $H_{\chi}$ such that $\rho(h) \neq 0$ ?

Let $\mathrm{C}_{\chi}$ denote the category of all $\mathfrak{g}$-modules on which $x-\chi(x)$ acts locally nilpotently for each $x \in \mathfrak{m}_{\chi}$. Given a $\mathfrak{g}$-module $M$ we denote by $\mathrm{Wh}(M)$ the subspace of $M$ consisting of all $m \in M$ such that $x . m=\chi(x) m$ for all $x \in \mathfrak{m}_{\chi}$. Of course, for $M \in \mathcal{C}_{\chi}$ we have $\mathrm{Wh}(M)=0$ if and only if $M=0$. Let $H_{\chi}$-mod denote the category of all left $H_{\chi}$-modules. In the Appendix to [31], Skryabin proved that the functor

$$
H_{\chi}-\bmod \rightarrow \mathcal{C}_{\chi}, \quad V \mapsto Q_{\chi} \otimes_{H_{\chi}} V,
$$

is an equivalence of categories. The inverse equivalence is given by the functor

$$
\mathrm{C}_{\chi} \rightarrow H_{\chi} \text {-mod, } \quad M \mapsto \mathrm{Wh}(M) ;
$$

see also [10, Sect. 6]. Skryabin's result implies that the $\mathfrak{g}$-module $Q_{\chi} \otimes_{H_{\chi}} V$ is simple if and only if so is the $H_{\chi}$-module $V$. By the Irreducibility Theorem, the associated variety of the annihilator in $U(\mathfrak{g})$ of any simple $\mathfrak{g}$-module coincides with the closure of a nilpotent orbit in $\mathfrak{g}^{*}$; see $[2,18,21,36,12]$. Our goal in this section is to determine the associated varieties of the annihilators $\operatorname{Ann}_{U(\mathfrak{g})} M$ for all $M \in \mathcal{C}_{\chi}$ with $\operatorname{dim} \mathrm{Wh}(M)<\infty$. Such modules are in 1-1 correspondence with the finite-dimensional representations of $H_{\chi}$. 
3.2. We recall the definition of the Gelfand-Kirillov dimension of a finitely generated $U(\mathfrak{g})$-module $M$. Firstly note that there exists a finite-dimensional subspace $M_{0} \subseteq M$ such that $M=\bigcup_{n>0} U_{n} M_{0}$ where $U_{n}$ stands for the $n$th component of the standard filtration of $U(\mathfrak{g})$. It is known that for all $n \gg 0$ the dimension of $U_{n} M_{0}$ is a polynomial in $n$. The Gelfand-Kirillov dimension of $M$, denoted $\operatorname{Dim}(M)$, is defined as the degree of this polynomial. The key point in this definition is that $\operatorname{Dim}(M)$ is independent of the choice of $M_{0}$; see [13, p. 134] for more details.

Now let $I$ be a two-sided ideal of the universal enveloping algebra $U(\mathfrak{g})$. The subspaces $I_{n}:=I \cap U_{n}$ with $n \in \mathbb{Z}_{+}$form an increasing filtration of $I$ satisfying $U_{m} I_{n} \subseteq$ $I_{m+n}$ for all $m, n \in \mathbb{Z}_{+}$. The associated graded algebra gr $I \hookrightarrow \operatorname{gr} U(\mathfrak{g}) \cong S(\mathfrak{g})$ is therefore identified with a homogeneous ideal of the symmetric algebra $S(\mathfrak{g})$ stable under the adjoint action of $G$. The associated variety $\mathcal{V A}(I)$ of the ideal $I$ is defined as the maximal spectrum of the affine algebra $S(\mathfrak{g}) / \mathrm{gr} I$. It is immediate from the definition that $\mathcal{V} \mathcal{A}(I)$ is a Zariski closed, conical, $G$-invariant subset of $\operatorname{Max} S(\mathfrak{g})=\mathfrak{g}^{*}$. For $M$ as above we have

$$
\operatorname{dim} \mathcal{V} \mathcal{A}\left(\operatorname{Ann}_{U(\mathfrak{g})} M\right) \leq 2 \operatorname{Dim}(M) ;
$$

see $[13,(10.7)$ and (17.11)]. The $\mathfrak{g}$-module $M$ is called holonomic if equality holds here, that is, $\operatorname{dim} \mathcal{V A}\left(\operatorname{Ann}_{U(\mathfrak{g})} M\right)=2 \operatorname{Dim}(M)$.

3.3. We are now in a position to state and prove the main result of this section.

Theorem 3.1. Let $M \in \mathcal{C}_{\chi}$ and $I=\operatorname{Ann}_{U(\mathfrak{g})} M$. Then the following hold:

(i) $\mathcal{O}_{\chi} \subset \mathcal{V} \mathcal{A}(I)$.

(ii) If $\operatorname{dim} \operatorname{Wh}(M)<\infty$, then $\operatorname{Dim}(M)=\frac{1}{2} \operatorname{dim} \mathcal{O}_{\chi}$ and $\mathcal{V A}(I)$ coincides with the Zariski closure of $\mathcal{O}_{\chi}$. In particular, $M$ is a holonomic $\mathfrak{g}$-module.

Proof. (1) Let $\top$ denote the anti-involution of the algebra $U(\mathfrak{g})$ such that $x^{\top}=-x$ and $(u v)^{\top}=v^{\top} u^{\top}$ for all $x \in \mathfrak{g}$ and all $u, v \in U(\mathfrak{g})$. Let $M^{*}$ denote the $\mathfrak{g}$-module dual to $M$. It is easy to see that $\operatorname{Ann}_{U(\mathfrak{g})} M^{*}=I^{\top}$. Since $\top$ preserves the standard filtration of $U(\mathfrak{g})$ and acts as a scalar operator on each factor space $U_{n} / U_{n-1}$ we have $\operatorname{grAnn}_{U(\mathfrak{g})} M^{*}=$ $\operatorname{gr}\left(I^{\top}\right)=\operatorname{gr} I$. Consequently, $\mathcal{V} \mathcal{A}\left(\operatorname{Ann}_{U(\mathfrak{g})} M^{*}\right)=\mathcal{V} \mathcal{A}(I)$.

Pick any nonzero $m \in \mathrm{Wh}(M)$ and view it as a linear function on $M^{*}$ via $m(f)=$ $f(m)$ for all $f \in M^{*}$. Then

$$
m(x . f)=(x \cdot f)(m)=-f(x \cdot m)=-\chi(x) f(m)=-\chi(x) m(f) \quad\left(\forall x \in \mathfrak{m}_{\chi}\right) .
$$

This shows that $m$ is a dual $\left(\mathfrak{m}_{\chi},-\chi\right)$-Whittaker vector of the $\mathfrak{g}$-module $M^{*}$; see [24, p. 221]. Thanks to Matumoto's theorem [24] we are now able to deduce that the associated variety of $\operatorname{Ann}_{U(\mathfrak{g})} M^{*}$ contains $-\chi$. As $\mathcal{V A}\left(\operatorname{Ann}_{U(\mathfrak{g})} M^{*}\right)=\mathcal{V} \mathcal{A}(I)$ is conical and $G$ stable this yields $\mathcal{O}_{\chi} \subset \mathcal{V} \mathcal{A}(I)$, proving (i).

(2) From now on suppose that $M_{0}:=\mathrm{Wh}(M)$ is finite-dimensional. Let $2 d=\operatorname{dim} \mathcal{O}_{\chi}$. By the $\mathfrak{s l}_{2}$-theory, $r=\operatorname{dim} \mathfrak{z}_{\chi}=\operatorname{dim} \mathfrak{g}(0)+\operatorname{dim} \mathfrak{g}(-1)=\operatorname{dim} \mathfrak{g}(0)+2 s$. Hence $d=$ $\operatorname{dim} \mathfrak{m}_{\chi}=\operatorname{dim} \mathfrak{p}_{e}-\operatorname{dim} \mathfrak{g}(0)-s=m-r+s$. Let $Y_{1}, \ldots, Y_{d}$ be a basis of $\mathfrak{m}_{\chi}$ with $Y_{i} \in \mathfrak{g}\left(-l_{i}-2\right)$ for some $l_{i} \geq-1$ and choose $X_{i} \in \mathfrak{g}\left(l_{i}\right)$ with $1 \leq i \leq d$ such that 
$\chi\left(\left[Y_{i}, X_{j}\right]\right)=\delta_{i j}$. No generality will be lost by assuming further that $X_{i}=z_{i}$ for $i \leq s$ and $X_{s+j}=x_{r+j}$ for $1 \leq j \leq m-r$, where $z_{i}$ and $x_{j}$ are basis vectors introduced in 2.1 For $\mathbf{a}=\left(a_{1}, \ldots, a_{d}\right) \in \mathbb{Z}_{+}^{d}$ put

$$
|\mathbf{a}|=\sum_{i=1}^{d} a_{i}, \quad \text { wt } \mathbf{a}=-\sum_{i=1}^{s} a_{i}+\sum_{i=1}^{m-r} n_{r+i} a_{s+i}, \quad X^{\mathbf{a}}=X_{1}^{a_{1}} \cdots X_{d}^{a_{d}} \in U_{|\mathbf{a}|} .
$$

(3) Let $\left\{m_{i}\right\}$ be a basis of $M_{0}$. Since the $U(\mathfrak{g})$-module $Q_{\chi}$ is generated by $1_{\chi}$ it follows from 3.1.1 and 3.1.2 that the $U(\mathfrak{g})$-module $M$ is generated by $M_{0}$. As explained in [31, p. 53] the vectors $X^{\mathbf{a}}\left(m_{i}\right)$ with $\mathbf{a} \in \mathbb{Z}_{+}^{d}$ and $i \leq \operatorname{dim} M_{0}$ are linearly independent. Therefore,

$$
\operatorname{dim} U_{n} M_{0} \geq\left(\operatorname{dim} M_{0}\right) \cdot \operatorname{Card}\left\{\mathbf{a} \in \mathbb{Z}_{+}^{d}|n \geq| \mathbf{a} \mid\right\}=\left(\operatorname{dim} M_{0}\right) \cdot\left(\begin{array}{c}
n+d \\
d
\end{array}\right) .
$$

For all $n \gg 0$ the LHS is a polynomial in $n$ of degree $\operatorname{Dim}(M)$, while the RHS is a polynomial in $n$ of degree $d$. This yields $\operatorname{Dim}(M) \geq d$.

(4) Now put $N=\max \{n \in \mathbb{Z} \mid \mathfrak{g}(n) \neq 0\}$ and let $M_{d, j}$ denote the subspace of $M$ spanned by all vectors $X^{\mathbf{a}}\left(m_{i}\right)$ with $|\mathbf{a}| \leq j$. We claim that

$$
U_{k} M_{0} \subseteq M_{d,(N+2) k} \quad\left(\forall k \in \mathbb{Z}_{+}\right) .
$$

For $\mathbf{k}=\left(k_{1}, \ldots, k_{r}\right) \in \mathbb{Z}_{+}^{r}$ set

$$
\text { wt } \mathbf{k}=\sum_{i=1}^{r} n_{i} k_{i}, \quad x^{\mathbf{k}}=x_{1}^{k_{1}} \cdots x_{r}^{k_{r}} \in U(\mathfrak{g}), \quad \Theta^{\mathbf{k}}=\Theta_{1}^{k_{1}} \cdots \Theta_{r}^{k_{r}} \in H_{\chi} .
$$

Note that wt $\mathbf{k} \geq 0$. Given $\mathbf{a} \in \mathbb{Z}_{+}^{d}$ and $\mathbf{b} \in \mathbb{Z}_{+}^{r}$ put $|(\mathbf{a} ; \mathbf{b})|_{e}:=\operatorname{wt} \mathbf{a}+\mathrm{wt} \mathbf{b}+2|\mathbf{a}|+2|\mathbf{b}|$. Using the formula in [31, p. 27] and the isomorphism $M \cong Q_{\chi} \otimes_{H_{\chi}} M_{0}$ it is easy to observe that

$$
X^{\mathbf{a}} x^{\mathbf{b}}\left(m_{i}\right)=\left(X^{\mathbf{a}} \Theta^{\mathbf{b}}+\sum_{|(\mathbf{i} ; \mathbf{j})|_{e}=|(\mathbf{a} ; \mathbf{b})|_{e},|\mathbf{i}+| \mathbf{j}|>| \mathbf{a}|+| \mathbf{b} \mid} \mu_{\mathbf{i}, \mathbf{j}} X^{\mathbf{i}} \Theta^{\mathbf{j}}+\sum_{|(\mathbf{i} ; \mathbf{j})|_{e}<|(\mathbf{a} ; \mathbf{b})|_{e}} \mu_{\mathbf{i}, \mathbf{j}} X^{\mathbf{i}} \Theta^{\mathbf{j}}\right)\left(m_{i}\right)
$$

for some $\mu_{\mathbf{i}, \mathbf{j}} \in \mathbb{k}$. As the subspace $U_{k} M_{0}$ is spanned by all $X^{\mathbf{a}} x^{\mathbf{b}}\left(m_{i}\right)$ with $|\mathbf{a}|+|\mathbf{b}| \leq k$, it is contained in the span of all $X^{\mathbf{i}}\left(m_{j}\right)$ such that

$$
\begin{aligned}
\text { wt } \mathbf{i} & \leq|(\mathbf{i} ; \mathbf{j})|_{e} \leq \max _{|\mathbf{a}|+|\mathbf{b}| \leq k}|(\mathbf{a} ; \mathbf{b})|_{e} \leq 2 k+\max _{|\mathbf{a}|+|\mathbf{b}| \leq k}(\text { wt } \mathbf{a}+\mathrm{wt} \mathbf{b}) \\
& \leq 2 k+\max _{|\mathbf{a}|+|\mathbf{b}| \leq k}(N|\mathbf{a}|+N|\mathbf{b}|) \leq(N+2) k .
\end{aligned}
$$

The claim follows. Since all vectors $X^{\mathbf{i}}\left(m_{j}\right)$ are linearly independent, we derive

$$
\begin{aligned}
\operatorname{dim} U_{k} M_{0} & \leq \operatorname{dim} M_{d,(N+2) k}=\left(\operatorname{dim} M_{0}\right) \cdot \operatorname{Card}\left\{\mathbf{i} \in \mathbb{Z}_{+}^{d}|(N+2) k \geq| \mathbf{i} \mid\right\} \\
& =\left(\operatorname{dim} M_{0}\right) \cdot\left(\begin{array}{c}
N k+2 k+d \\
d
\end{array}\right) .
\end{aligned}
$$


Since the RHS is a polynomial in $k$ of degree $d$, we get $\operatorname{Dim}(M) \leq d$. In conjunction with part (3) this shows that $\operatorname{Dim}(M)=d=\left(\operatorname{dim} \mathcal{O}_{\chi}\right) / 2$.

(5) Since the $H_{\chi}$-module $\mathrm{Wh}(M)$ is finite-dimensional, Skryabin's equivalence of categories described in 3.1 implies that the $\mathfrak{g}$-module $M$ has a composition series $M=M_{1} \supset$ $\ldots \supset M_{l} \supset M_{l+1}=0$ such that $M_{i} / M_{i+1} \in \mathcal{C}_{\chi}$ and $\operatorname{dim} \mathrm{Wh}\left(M_{i} / M_{i+1}\right)<\infty$ for all $i \leq l$. Set $J_{i}:=\operatorname{Ann}_{U(\mathfrak{g})}\left(M_{i} / M_{i+1}\right)$. It is immediate from the discussion in [14, (17.7)] that

$$
\sqrt{\operatorname{gr} I}=\bigcap_{i=1}^{l} \sqrt{\operatorname{gr} J_{i}} .
$$

On the other hand, it follows from 3.2.1 and parts (1) and (4) of this proof that for any $\mathfrak{g}$-module $N \in \mathcal{C}_{\chi}$ with $\operatorname{dim} \operatorname{Wh}(N)<\infty$ one has

$$
\operatorname{dim} \mathcal{O}_{\chi} \leq \operatorname{dim} \mathcal{V} \mathcal{A}\left(\operatorname{Ann}_{U(\mathfrak{g})} N\right) \leq 2 \operatorname{Dim}(N)=\operatorname{dim} \mathcal{O}_{\chi}
$$

In conjunction with the Irreducibility Theorem mentioned in 3.1 this shows that $\mathcal{V A}\left(M_{i} / M_{i+1}\right)$ coincides with the Zariski closure $\overline{\mathcal{O}}_{\chi}$ for all $i \leq l$. But then 3.3.1) yields $\mathcal{V} \mathcal{A}(I)=\overline{\mathcal{O}}_{\chi}$, completing the proof.

3.4. Recall that a two-sided ideal $I$ of $U(\mathfrak{g})$ is called completely prime (respectively, primitive) if $U(\mathfrak{g}) / I$ is a domain (respectively, if $I$ is the annihilator of a simple $\mathfrak{g}$ module). For $n \in \mathbb{Z}_{+}$the set $Y_{n}:=\left\{\psi \in \mathfrak{g}^{*} \mid \operatorname{dim}\left(\operatorname{Ad}^{*} G\right) \psi=n\right\}$ is locally closed in the Zariski topology of $\mathfrak{g}^{*}$. A (locally closed) subset of $\mathfrak{g}^{*}$ is called a sheet if it coincides with an irreducible component of one of the locally closed sets $Y_{n}$. It is well-known that each sheet is $G$-invariant and contains a unique nilpotent coadjoint orbit (such an orbit may lie in several sheets, however).

Conjecture 3.1. Let $e$ be an arbitrary nilpotent element in $\mathfrak{g}$ and let $\chi=\chi_{e}$ be the corresponding linear function on $\mathfrak{g}$.

1. The algebra $H_{\chi}$ contains an ideal of codimension 1 .

2. The ideals of codimension 1 in $H_{\chi}$ are finite in number if and only if $\mathcal{O}_{\chi}$ is a sheet in $\mathfrak{g}^{*}$.

3. For any ideal $I$ of codimension 1 in $H_{\chi}$ the ideal $\tilde{I}=\operatorname{Ann}_{U(\mathfrak{g})}\left(Q_{\chi} \otimes_{H_{\chi}} H_{\chi} / I\right)$ of $U(\mathfrak{g})$ is completely prime.

Our last conjecture provides a hypothetical converse to Theorem 3.1(ii). It indicates that each category $\mathcal{C}_{\chi}$ is potentially very important for the theory of primitive ideals.

Conjecture 3.2. Let $\chi$ be as above and let $I$ be a primitive ideal of $U(\mathfrak{g})$ whose associated variety equals $\overline{\mathcal{O}}_{\chi}$. Then there exists a simple $\mathfrak{g}$-module $M \in \mathcal{C}_{\chi}$ with $\operatorname{dim} \operatorname{Wh}(M)<$ $\infty$ such that $I=\operatorname{Ann}_{U(\mathfrak{g})} M$. 


\section{Minimal nilpotent algebras: a quadratic relation}

4.1. Let $\mathfrak{h}$ be a Cartan subalgebra of $\mathfrak{g}$, and let $\Phi$ be the root system of $\mathfrak{g}$ relative to $\mathfrak{h}$. Let $\left\{e_{\alpha} \mid \alpha \in \Phi\right\} \cup\left\{h_{\alpha} \mid \alpha \in \Phi\right\}$ be a Chevalley system in $\mathfrak{g}$ with each triple $\left(e_{\alpha}, h_{\alpha}, e_{-\alpha}\right)$ being an $\mathfrak{s l}_{2}$-triple in $\mathfrak{g}$. Let $\Pi=\left\{\alpha_{1}, \ldots, \alpha_{\ell}\right\}$ be a basis of simple roots in $\Phi$ with the elements in $\Pi$ numbered as in [4], and let $\left\{\varpi_{1}, \ldots, \varpi_{\ell}\right\}$ be the corresponding system of fundamental weights in $\mathfrak{h}^{*}$. Let $\Phi^{+}$and $\Phi^{-}$be the positive and the negative system of $\Phi$ relative to $\Pi$, respectively, and let $P$ denote the lattice of integral weights in $\mathfrak{h}^{*}$. As usual, given $\lambda, \mu \in P$ we write $\lambda \geq \mu$ if and only if $\lambda-\mu$ is a sum of positive roots. Let $P^{+}=\left\{\sum_{i} a_{i} \varpi_{i} \mid i \in \mathbb{Z}_{+}\right\}$, the set of dominant weights, and $\rho=\varpi_{1}+\cdots+\varpi_{\ell}=$ $\frac{1}{2} \sum_{\alpha \in \Phi^{+}} \alpha$, the half-sum of positive roots. Let $W$ be the Weyl group of the root system $\Phi$; it is generated by reflections $s_{\alpha}$ with $\alpha \in \Phi$. The dot action of $W$ on $\mathfrak{h}^{*}$ is defined by setting $w \cdot \lambda=w(\lambda+\rho)-\rho$ for all $w \in W$ and $\lambda \in \mathfrak{h}^{*}$.

If $\mathfrak{g}$ is not of type $\mathrm{A}$ or $\mathrm{C}$, there is a unique long root in $\Pi$ linked with the lowest root $-\widetilde{\alpha} \in \Phi^{-}$on the extended Dynkin diagram of $\mathfrak{g}$; we call it $\beta$. For $\mathfrak{g}$ of type $\mathrm{A}_{n}$ and $\mathrm{C}_{n}$ put $\beta=\alpha_{n}$. In this paper, we will be mostly concerned with the $\mathfrak{s l}_{2}$-triple $(e, h, f)=$ $\left(e_{\beta}, h_{\beta}, e_{-\beta}\right)$. Recall that the invariant form $(\cdot, \cdot)$ on $\mathfrak{g}$ has the property that $(e, f)=1$. This entails $(h, h)=2$. It is well-known that the restriction of $(\cdot, \cdot)$ to $\mathfrak{h}$ is nondegenerate and induces a $W$-invariant scalar product on the $\mathbb{Q}$-span of $P$ in $\mathfrak{h}^{*}$. More precisely, for all $\lambda, \mu \in \mathfrak{h}^{*}$ we have $(\lambda, \mu)=\left(t_{\lambda}, t_{\mu}\right)$ where $t_{\lambda}, t_{\mu} \in \mathfrak{h}$ are such that $\lambda=\left(t_{\lambda}, \cdot\right)$ and $\mu=\left(t_{\mu}, \cdot\right)$. Put $\langle\lambda, \alpha\rangle=2(\lambda, \alpha) /(\alpha, \alpha)$ for all $\lambda \in \mathfrak{h}^{*}$ and $\alpha \in \Phi$. Since $(\cdot, \cdot)$ is a multiple of the Killing form of $\mathfrak{g}$, there is a constant $c \in \mathbb{k}^{\times}$such that $\beta(x)=c\left(h_{\beta}, x\right)$ for all $x \in \mathfrak{h}$. The equality $\beta\left(h_{\beta}\right)=2=\left(h_{\beta}, h_{\beta}\right)$ now shows that $c=1$ and $t_{\beta}=h_{\beta}$. Hence $(\gamma, \gamma)=2$ for all long roots $\gamma \in \Phi$.

From now on we assume that $\chi=(e, \cdot)$ where $e=e_{\beta}$. It is well-known that the adjoint action of $h=h_{\beta}$ on $\mathfrak{g}$ gives rise to a short $\mathbb{Z}$-grading

$$
\mathfrak{g}=\mathfrak{g}(-2) \oplus \mathfrak{g}(-1) \oplus \mathfrak{g}(0) \oplus \mathfrak{g}(1) \oplus \mathfrak{g}(2)
$$

with $\mathfrak{g}(i)=\{x \in \mathfrak{g} \mid[h, x]=i x\}$ for all $i \in \mathbb{Z}$ and with $\mathfrak{g}(1) \oplus \mathfrak{g}(2) \cong \mathfrak{g}(-1) \oplus \mathfrak{g}(-2)$ isomorphic to a Heisenberg Lie algebra. We also have $\mathfrak{g}(2)=\mathbb{k} e, \mathfrak{g}(-2)=\mathbb{k} f$, and $\mathfrak{g}(0)=\mathfrak{c}_{\mathfrak{g}}(h)$. The $\mathfrak{s l}_{2}$-theory implies that

$$
\mathfrak{c}_{\mathfrak{g}}(e)=\mathfrak{g}(0)^{\sharp} \oplus \mathfrak{g}(1) \oplus \mathfrak{g}(2)
$$

where $\mathfrak{g}(0)^{\sharp}=\{x \in \mathfrak{g}(0) \mid[x, e]=0\}$. More importantly for our later deliberations, $\mathfrak{g}(0)^{\sharp}$ is the orthogonal complement to $\mathbb{k} h$ in $\mathfrak{g}(0)$ and hence coincides with the image of the Lie algebra endomorphism

$$
\sharp: \mathfrak{g}(0) \rightarrow \mathfrak{g}(0), \quad x \mapsto x-\frac{1}{2}(x, h) h .
$$

In particular, $\mathfrak{g}(0)^{\sharp}$ is an ideal of codimension 1 in the Levi subalgebra $\mathfrak{g}(0)$. It is wellknown that outside type A the centre of $\mathfrak{g}(0)$ coincides with $\mathbb{k} h$ and $\mathfrak{g}(1)$ is an irreducible $\operatorname{ad} \mathfrak{g}(0)^{\sharp}$-module. As a consequence, if $\mathfrak{g}$ is not of type $A$, then $\mathfrak{g}(0)^{\sharp}=[\mathfrak{g}(0), \mathfrak{g}(0)]$ is the only ideal of codimension 1 in $\mathfrak{g}(0)$ and $\mathfrak{c}_{\mathfrak{g}}(e)=\left[\mathfrak{c}_{\mathfrak{g}}(e), \mathfrak{c}_{\mathfrak{g}}(e)\right]$ is a perfect Lie algebra. 
Note that in the present case $\mathfrak{g}(0)^{\sharp}=\operatorname{Lie} C(e)$. Put $\mathfrak{h}_{e}:=\mathfrak{h} \cap \mathfrak{c}_{\mathfrak{g}}(e)$, a Cartan subalgebra in $\mathfrak{g}(0)^{\sharp}$. It can be assumed without loss of generality that $\mathfrak{h}_{e}=$ Lie $T_{e}$ where $T_{e}$ is as in Section 2. We can choose $z_{1}, \ldots, z_{s}, z_{s+1}, \ldots, z_{2 s}$ to be root vectors for $\mathfrak{h}$. Moreover, we can (and will) assume that the $z_{i}$ 's with $1 \leq i \leq s$ are root vectors in $\mathfrak{g}(-1)$ corresponding to negative roots $\gamma_{i} \in \Phi^{-}$. Then each $z_{i}^{*}$ with $1 \leq i \leq s$ is a root vector in $\mathfrak{g}(-1)$ corresponding to $\gamma_{i}^{*}:=-\beta-\gamma_{i} \in \Phi^{+}$.

4.2. Set $H:=H_{\chi}$ and identify $H$ with $\tilde{\mu}(H)$. Given $a \in \mathfrak{z}_{\chi}(0)$ and $w \in \mathfrak{z}_{\chi}(1)$ we define the following elements of $\mathbf{A}_{e}$ :

$$
\psi_{a}:=\frac{1}{2} \sum_{i=1}^{2 s}\left[a, z_{i}^{*}\right] z_{i}, \quad \varphi_{w}:=\frac{1}{3} \sum_{i, j=1}^{2 s}\left[w z_{i}^{*} z_{j}^{*}\right] z_{j} z_{i}+z_{w} .
$$

Recall that $\sum_{i=1}^{2 s}\left[w, z_{i}^{*}\right] z_{i}=-\sum_{i=1}^{2 s}\left[w, z_{i}\right] z_{i}^{*}$. The computation used in the proof of Lemma 2.5 shows that

$$
\left[z_{k}^{*}, \varphi_{w}\right]=\sum_{i=1}^{2 s}\left[w z_{i}^{*} z_{k}^{*}\right] z_{i}
$$

Notice that in $U\left(\mathfrak{p}_{e}\right) \otimes \mathbf{A}_{e}^{\text {op }}$ we have $\sum_{i=1}^{2 s}\left[w, z_{i}^{*}\right] \otimes z_{i}=-\sum_{i=1}^{2 s}\left[w, z_{i}\right] \otimes z_{i}^{*}$, and $[a \otimes f, b \otimes g]=[a, b] \otimes g f-b a \otimes[f, g] \quad\left(\forall a, b \in U\left(\mathfrak{p}_{e}\right), \forall f, g \in \mathbf{A}_{e}\right)$.

Keeping this in mind it is straightforward to see that for all $u, v \in \mathfrak{z} \chi(1)$,

$$
\begin{aligned}
{\left[\Theta_{u}, \Theta_{v}\right]=} & {\left[u \otimes 1+\sum_{i=1}^{2 s}\left[u, z_{i}^{*}\right] \otimes z_{i}+1 \otimes \varphi_{u}, v \otimes 1+\sum_{i=1}^{2 s}\left[v, z_{i}^{*}\right] \otimes z_{i}+1 \otimes \varphi_{v}\right] } \\
= & {[u, v] \otimes 1+\sum_{i=1}^{2 s}\left[[u, v], z_{i}^{*}\right] \otimes z_{i}+\sum_{i, j=1}^{2 s}\left[u, z_{i}\right] \otimes\left[z_{i}^{*}, \varphi_{v}\right] } \\
& -\sum_{i, j=1}^{2 s}\left[v, z_{i}\right] \otimes\left[z_{i}^{*}, \varphi_{u}\right]+\sum_{i, j=1}^{2 s}\left[\left[u, z_{i}^{*}\right],\left[v, z_{j}^{*}\right]\right] \otimes z_{j} z_{i} \\
& +\sum_{i=1}^{2 s}\left[v, z_{i}^{*}\right]\left[u, z_{i}\right] \otimes 1-1 \otimes\left[\varphi_{u}, \varphi_{v}\right]
\end{aligned}
$$

In view of 4.2.1 and 2.5.3 we have

$$
\begin{aligned}
& \sum_{i=1}^{2 s}\left[x, z_{i}\right] \otimes\left[z_{i}^{*}, \varphi_{y}\right]=\sum_{i, j=1}^{2 s}\left[x, z_{i}\right] \otimes\left[y z_{j}^{*} z_{i}^{*}\right] z_{j}=\sum_{i, j, k}\left[x, z_{i}\right] \otimes\left\langle z_{k}^{*},\left[y z_{j}^{*} z_{i}^{*}\right]\right\rangle z_{k} z_{j} \\
& \quad=\sum_{i, j, k}\left[x,\left\langle z_{i}^{*},\left[y z_{j}^{*} z_{k}^{*}\right]\right\rangle z_{i}\right] \otimes z_{k} z_{j}+\sum_{i, j, k}\left[x, z_{i}\right] \otimes z_{k}\left\langle z_{j}^{*},\left[y,\left[z_{i}^{*}, z_{k}^{*}\right]\right]\right\rangle z_{j} \\
& \quad=\sum_{i, j=1}^{2 s}\left[x,\left[y z_{j}^{*} z_{i}^{*}\right]\right] \otimes z_{i} z_{j}+\sum_{i=1}^{2 s}\left[x, z_{i}^{*}\right] \otimes z_{i}[y, f]
\end{aligned}
$$


for all $x, y \in \mathfrak{z}_{x}(1)$. Combining this with 4 4.2.2) and taking into account that

$$
\sum_{i, j=1}^{2 s}\left[\left[u, z_{i}^{*}\right],\left[v, z_{j}^{*}\right]\right] \otimes z_{i} z_{j}=\sum_{i, j=1}^{2 s}\left[\left[u, z_{i}^{*}\right],\left[v, z_{j}^{*}\right]\right] \otimes z_{j} z_{i}-\sum_{i=1}^{2 s}\left[\left[u, z_{i}\right],\left[v, z_{j}^{*}\right]\right],
$$

we now obtain

$$
\begin{aligned}
{\left[\Theta_{u}, \Theta_{v}\right]=} & {[u, v] \otimes 1+\sum_{i=1}^{2 s}\left[[u, v], z_{i}^{*}\right] \otimes z_{i}-\sum_{i, j=1}^{2 s}\left[v,\left[u z_{j}^{*} z_{i}^{*}\right]\right] \otimes z_{i} z_{j} } \\
& -\sum_{i=1}^{2 s}\left[v, z_{i}^{*}\right] \otimes z_{i}[u, f]+\sum_{i, j=1}^{2 s}\left[u,\left[v z_{j}^{*} z_{i}^{*}\right]\right] \otimes z_{i} z_{j}+\sum_{i=1}^{2 s}\left[u, z_{i}^{*}\right] \otimes z_{i}[v, f] \\
& +\sum_{i, j=1}^{2 s}\left[\left[u, z_{i}^{*}\right],\left[v, z_{j}^{*}\right]\right] \otimes z_{j} z_{i}+\sum_{i=1}^{2 s}\left[v, z_{i}^{*}\right]\left[u, z_{i}\right] \otimes 1-1 \otimes\left[\varphi_{u}, \varphi_{v}\right] \\
= & (f,[u, v])\left(e \otimes 1+\sum_{i=1}^{2 s}\left[e, z_{i}^{*}\right] \otimes z_{i}+\sum_{i, j=1}^{2 s}\left[e z_{i}^{*} z_{j}^{*}\right] \otimes z_{j} z_{i}\right) \\
& -\sum_{i, j=1}^{2 s}\left[\left[u, z_{i}^{*}\right],\left[v, z_{j}^{*}\right]\right] \otimes z_{j} z_{i}+\sum_{i=1}^{2 s}\left(\left[u, z_{i}^{*}\right] \otimes z_{i}[v, f]-\left[v, z_{i}^{*}\right] \otimes z_{i}[u, f]\right) \\
& +\sum_{i=1}^{2 s}\left[u, z_{i}\right]\left[v, z_{i}^{*}\right] \otimes 1-1 \otimes\left[\varphi_{u}, \varphi_{v}\right] .
\end{aligned}
$$

Next observe that $\left[a, z_{i}^{*}\right]^{\sharp}=\left[a, z_{i}\right]-\frac{1}{2}\left(h,\left[a, z_{i}^{*}\right]\right) h$, and

$$
\sum_{i=1}^{2 s}\left(h,\left[a, z_{i}^{*}\right]\right) z_{i}=\sum_{i=1}^{2 s}\left(e,\left[f,\left[a, z_{i}^{*}\right]\right]\right) z_{i}=\sum_{i=1}^{2 s}\left\langle z_{i}^{*},[a, f]\right\rangle z_{i}=[a, f]
$$

for all $a \in \mathfrak{z}_{\chi}(1)$. Since $\sum_{i=1}^{2 s} z_{i}^{*} z_{i}=s$ (as elements in $\mathbf{A}_{e}$ ), it follows from 4.2.3 that

$$
\begin{aligned}
\sum_{i, j=1}^{2 s}\left[x, z_{i}\right]^{\sharp} \otimes\left[\left[y, z_{i}^{*}\right]^{\sharp}, z_{j}^{*}\right] z_{j}=\sum_{i, j=1}^{2 s}\left[x, z_{i}\right]^{\sharp} \otimes\left[y z_{i}^{*} z_{j}^{*}\right] z_{j}+\frac{s}{2} \sum_{i=1}^{2 s}\left(h,\left[y, z_{i}^{*}\right]\right)\left[x, z_{i}\right]^{\sharp} \otimes 1 \\
=\sum_{i, j=1}^{2 s}\left[x, z_{i}\right] \otimes\left[y z_{i}^{*} z_{j}^{*}\right] z_{j}-\frac{1}{2} \sum_{i, j=1}^{2 s}\left(h,\left[x, z_{i}^{*}\right]\right) h \otimes\left[y z_{i}^{*} z_{j}^{*}\right] z_{j}+\frac{s}{2}[x,[y, f]]^{\sharp} \otimes 1 \\
=\frac{s}{2}[x,[y, f]]^{\sharp} \otimes 1+\sum_{i, j=1}^{2 s}\left[x, z_{i}\right] \otimes\left[y z_{j}^{*} z_{i}^{*}\right] z_{i}+\sum_{i=1}^{2 s}\left[x, z_{i}^{*}\right] \otimes[y, f] z_{i} \\
\quad+\frac{1}{2} \sum_{i=1}^{2 s} h \otimes\left[[y,[x, f]], z_{j}^{*}\right] z_{j}=\frac{s}{2}[x,[y, f]]^{\sharp} \otimes 1+\frac{1}{2} \sum_{i=1}^{2 s} h \otimes\left[[y,[x, f]], z_{i}^{*}\right] z_{i} \\
\quad+\sum_{i, j=1}^{2 s}\left[x,\left[y z_{j}^{*} z_{i}^{*}\right]\right] \otimes z_{i} z_{j}+\sum_{i=1}^{2 s}\left[x, z_{i}^{*}\right] \otimes z_{i}[y, f]+\sum_{i=1}^{2 s}\left[x, z_{i}^{*}\right] \otimes[y, f] z_{i}
\end{aligned}
$$


for all $x, y \in \mathfrak{z}_{x}(1)$. But then

$$
\begin{aligned}
\sum_{i, j=1}^{2 s}\left[x, z_{i}\right]^{\sharp} \otimes\left[\left[y, z_{i}^{*}\right]^{\sharp}, z_{j}^{*}\right] z_{j}+\sum_{i, j=1}^{2 s}\left[y, z_{i}^{*}\right]^{\sharp} \otimes\left[\left[x, z_{i}\right]^{\sharp}, z_{j}^{*}\right] z_{j} \\
=\sum_{i, j=1}^{2 s}\left[x, z_{i}\right]^{\sharp} \otimes\left[\left[y, z_{i}^{*}\right]^{\sharp}, z_{j}^{*}\right] z_{j}-\sum_{i, j=1}^{2 s}\left[y, z_{i}\right]^{\sharp} \otimes\left[\left[x, z_{i}^{*}\right]^{\sharp}, z_{j}^{*}\right] z_{j} \\
=(f,[x, y])\left(\frac{s}{2} h^{\sharp} \otimes 1-\frac{1}{2} h \otimes \sum_{i=1}^{2 s}\left[h, z_{i}\right]^{*} z_{i}+\sum_{i, j=1}^{2 s}\left[e z_{i}^{*} z_{j}^{*}\right] \otimes z_{j} z_{i}\right) \\
\quad-2 \sum_{i, j=1}^{2 s}\left[\left[x, z_{i}^{*}\right],\left[y, z_{j}^{*}\right]\right] \otimes z_{j} z_{i}-\sum_{i=1}^{2 s}\left[\left[x, z_{i}\right],\left[y, z_{i}^{*}\right]\right] \otimes 1 \\
\quad+2 \sum_{i=1}^{2 s}\left(\left[x, z_{i}^{*}\right] \otimes z_{i}[y, f]-\left[y, z_{i}^{*}\right] \otimes z_{i}[x, f]\right)-[[x, y], f] \otimes 1 .
\end{aligned}
$$

We used the fact that

$$
\sum_{i=1}^{2 s}\left[x, z_{i}^{*}\right] \otimes\left[[y, f], z_{i}\right]=\sum_{i=1}^{2 s}\left[x, z_{i}\right] \otimes\left\langle z_{i}^{*},[y, f]\right\rangle=-[x,[y, f]] \otimes 1 .
$$

To ease notation, set

$$
A(u, v):=\left[\Theta_{u}, \Theta_{v}\right]-\frac{1}{2} \sum_{i=1}^{2 s}\left(\Theta_{[u, z i]^{\sharp}} \Theta_{\left[v, z_{i}^{*}\right]^{\sharp}}+\Theta_{\left[v, z_{i}^{*}\right]^{\sharp}} \Theta_{\left[u, z_{i}\right]^{\sharp}}\right) .
$$

Note that

$$
\begin{aligned}
& \sum_{i=1}^{2 s}\left(\Theta_{\left[u, z_{i}\right]^{\sharp}} \Theta_{\left[v, z_{i}^{*}\right]^{\sharp}}+\Theta_{\left[v, z_{i}^{*}\right]^{\sharp}} \Theta_{\left[u, z_{i}\right]^{\sharp}}\right)=\sum_{i=1}^{2 s}\left(\left[u, z_{i}\right]^{\sharp}\left[v, z_{i}^{*}\right]^{\sharp}+\left[v, z_{i}^{*}\right]^{\sharp}\left[u, z_{i}\right]^{\sharp}\right) \otimes 1 \\
& +\sum_{i, j=1}^{2 s}\left[u, z_{i}\right]^{\sharp} \otimes\left[\left[v, z_{i}^{*}\right]^{\sharp}, z_{j}^{*}\right] z_{j} z_{i}+\sum_{i, j=1}^{2 s}\left[v, z_{i}^{*}\right]^{\sharp} \otimes\left[\left[u, z_{i}\right]^{\sharp}, z_{j}^{*}\right] z_{j} z_{i}+1 \otimes \psi(u, v),
\end{aligned}
$$

where $\psi(u, v)=\frac{1}{4} \sum_{i=1}^{2 s}\left(\psi_{\left[v, z_{i}^{*}\right]^{\sharp}} \psi_{\left[u, z_{i}\right]^{\sharp}}+\psi_{\left[u, z_{i}\right]^{\sharp}} \psi_{\left[v, z_{i}^{*}\right]^{\sharp}}\right) \in \mathbf{A}_{e}$. Since

$$
\sum_{i=1}^{2 s}\left(x, z_{i}^{*}\right) z_{i}=-\sum_{i=1}^{2 s}\left(x, z_{i}\right) z_{i}^{*}=[x, f] \quad(\forall x \in \mathfrak{z} \chi(1)),
$$

we have

$$
\begin{aligned}
\sum_{i=1}^{2 s}\left(\left[u, z_{i}\right]^{\sharp}\left[v, z_{i}^{*}\right]^{\sharp}+\left[v, z_{i}^{*}\right]^{\sharp}\left[u, z_{i}\right]^{\sharp}\right)=\sum_{i=1}^{2 s}\left(\left[u, z_{i}\right]\left[v, z_{i}^{*}\right]+\left[v, z_{i}^{*}\right]\left[u, z_{i}\right]\right) \\
-\sum_{i=1}^{2 s}\left(\left(u, z_{i}\right) h\left[v, z_{i}^{*}\right]+\left(v, z_{i}^{*}\right) h\left[u, z_{i}\right]-\frac{1}{4}\left(u, z_{i}\right)\left(v, z_{i}^{*}\right) h^{2}-\frac{1}{4}\left(v, z_{i}^{*}\right)\left(u, z_{i}\right) h^{2}\right) \\
=-\frac{1}{2}(e,[u, v]) h^{2}+2 \sum_{i=1}^{2 s}\left[u, z_{i}\right]\left[v, z_{i}^{*}\right]-\sum_{i=1}^{2 s}\left[\left[u, z_{i}\right],\left[v, z_{i}^{*}\right]\right] .
\end{aligned}
$$


Since $h^{\sharp}=0$ and $h \otimes \sum_{i=1}^{2 s}\left[h, z_{i}^{*}\right] z_{i}=-s h \otimes 1$, we now combine the above with 4.2.4 and 4.2 .5 to deduce that

$$
\begin{aligned}
A(u, v)= & \frac{1}{2}(e,[u, v])\left(\frac{4 e+h^{2}-(s+2) h}{2} \otimes 1+2 \sum_{i=1}^{2 s}\left[e, z_{i}^{*}\right] \otimes z_{i}\right. \\
& \left.+\sum_{i, j=1}^{2 s}\left[e z_{i}^{*} z_{j}^{*}\right] \otimes z_{j} z_{i}\right)+1 \otimes\left(\left[\varphi_{v}, \varphi_{u}\right]-\frac{1}{2} \psi(u, v)\right) .
\end{aligned}
$$

4.3. Recall that $\mathfrak{z} \chi(0)=\mathfrak{g}(0)^{\sharp}$ is an ideal of codimension 1 in the Levi subalgebra $\mathfrak{g}(0)=\mathfrak{c}_{\mathfrak{g}}(h)$ of $\mathfrak{g}$. Let $\left\{a_{i}\right\}$ and $\left\{b_{i}\right\}$ be dual bases of $\mathfrak{z}_{\chi}(0)$ with respect to the restriction of $(\cdot, \cdot)$ to $\mathfrak{z}_{\chi}(0)$, and let $C_{0}=\sum_{i} a_{i} b_{i}$ be the corresponding Casimir element of $U\left(\mathfrak{z}_{\chi}(0)\right)$. Set $\Theta_{\text {Cas }}:=\sum_{i} \Theta_{a_{i}} \Theta_{b_{i}}$, an element of $H$. Although $\Theta_{\text {Cas }}$ is not central in $H$, Lemma 2.4 shows that it commutes with all operators $\Theta_{x}$ for $x \in \mathfrak{z} \chi(0)$. Since the skew-symmetric form $\langle\cdot, \cdot\rangle$ is invariant under $\mathfrak{z}_{\chi}(0)$ and $\mathbb{k} h$ is orthogonal to $\mathfrak{g}(0)^{\sharp}$ with respect to $(\cdot, \cdot)$, we have

$$
\begin{aligned}
\sum_{i, j=1}^{2 s}\left[e z_{i}^{*} z_{j}^{*}\right]^{\sharp} \otimes z_{j} z_{i} & \left.\left.=\sum_{i, j, k}\left(b_{k},\left[e z_{i}^{*} z_{j}^{*}\right]^{\sharp}\right]\right) a_{k} \otimes z_{j} z_{i}=\sum_{i, j, k}\left(b_{k},\left[e z_{i}^{*} z_{j}^{*}\right]\right]\right) a_{k} \otimes z_{j} z_{i} \\
& =\sum_{i, j, k}\left(\left[b_{k} z_{j}^{*} z_{i}^{*}\right], e\right) a_{k} \otimes z_{j} z_{i}=-\sum_{i, j, k}\left\langle z_{i}^{*},\left[b_{k} z_{j}^{*}\right]\right\rangle a_{k} \otimes z_{j} z_{i} \\
& =-\sum_{i, j, k}\left\langle z_{j}^{*},\left[b_{k} z_{i}^{*}\right]\right\rangle a_{k} \otimes z_{j} z_{i}=-\sum_{k, i} a_{k} \otimes\left[b_{k}, z_{i}^{*}\right] z_{i} .
\end{aligned}
$$

Interchanging the rôles of $\left\{a_{i}\right\}$ and $\left\{b_{i}\right\}$ we now obtain

$$
\sum_{i, j=1}^{2 s}\left[e z_{i}^{*} z_{j}^{*}\right]^{\sharp} \otimes z_{j} z_{i}=-\sum_{k, i} b_{k} \otimes\left[a_{k}, z_{i}^{*}\right] z_{i} .
$$

Next observe that

$$
\begin{aligned}
\sum_{i, j=1}^{2 s}\left[e z_{i}^{*} z_{j}^{*}\right]^{\sharp} \otimes z_{j} z_{i} & =\sum_{i, j=1}^{2 s}\left[e z_{i}^{*} z_{j}^{*}\right] \otimes z_{j} z_{i}-\frac{1}{2} \sum_{i, j=1}^{2 s}\left(h,\left[e z_{i}^{*} z_{j}^{*}\right]\right) h \otimes z_{j} z_{i} \\
& =\sum_{i, j=1}^{2 s}\left[e z_{i}^{*} z_{j}^{*}\right] \otimes z_{j} z_{i}+\frac{1}{2} \sum_{i, j=1}^{2 s}\left(\left[\left[h, z_{j}\right], z_{i}^{*}\right], e\right) h \otimes z_{j}^{*} z_{i} \\
& =\sum_{i, j=1}^{2 s}\left[e z_{i}^{*} z_{j}^{*}\right] \otimes z_{j} z_{i}+\frac{s h}{2} \otimes 1 .
\end{aligned}
$$

It follows that

$$
\sum_{i, j=1}^{2 s}\left[e z_{i}^{*} z_{j}^{*}\right] \otimes z_{j} z_{i}=-\frac{s h}{2} \otimes 1-\frac{1}{2}\left(\sum_{i, j} a_{i} \otimes\left[b_{i}, z_{j}^{*}\right] z_{j}+\sum_{i, j} b_{i} \otimes\left[a_{i}, z_{j}^{*}\right] z_{j}\right) .
$$


As a result,

$$
\begin{aligned}
\Theta_{\mathrm{Cas}} & =\sum_{i} a_{i} b_{i} \otimes 1+\frac{1}{2} \sum_{i, j}\left(a_{i} \otimes\left[b_{i}, z_{j}^{*}\right] z_{j}+b_{i} \otimes\left[a_{i}, z_{j}^{*}\right] z_{j}\right)+1 \otimes c_{0}^{\prime} \\
& =\left(-\frac{s h}{2}+\sum_{i} a_{i} b_{i}\right) \otimes 1-\sum_{i, j=1}^{2 s}\left[e z_{i}^{*} z_{j}^{*}\right] \otimes z_{j} z_{i}+1 \otimes c_{0}^{\prime},
\end{aligned}
$$

where $c_{0}^{\prime}=\frac{1}{4} \sum_{i, j, k}\left[b_{i}, z_{j}^{*}\right] z_{j}\left[a_{i}, z_{k}^{*}\right] z_{k} \in \mathbf{A}_{e}$.

4.4. Let $C$ denote the Casimir element of $U(\mathfrak{g})$ corresponding to the invariant form $(\cdot, \cdot)$. Clearly, $C$ induces a $\mathfrak{g}$-endomorphism of $\widehat{Q}_{\chi}$, and hence can be viewed as a central element of $H$. To determine $\widetilde{\mu}(C)$ we first observe that the (ordered) sets

$$
\{e, h, f\} \cup\left\{a_{i}\right\} \cup\left\{\left[e, z_{i}^{*}\right] \mid 1 \leq i \leq 2 s\right\} \cup\left\{z_{i} \mid 1 \leq i \leq 2 s\right\}
$$

and

$$
\{f, h / 2, e\} \cup\left\{b_{i}\right\} \cup\left\{z_{i} \mid 1 \leq i \leq 2 s\right\} \cup\left\{\left[e, z_{i}^{*}\right] \mid 1 \leq i \leq 2 s\right\}
$$

are dual bases of $\mathfrak{g}$ with respect to $(\cdot, \cdot)$. Since

$$
\sum_{i=1}^{2 s}\left[z_{i},\left[e, z_{i}^{*}\right]\right]=\sum_{i=1}^{2 s}\left(\left[\left[z_{i}, e\right], z_{i}^{*}\right]+\left[e,\left[z_{i}, z_{i}^{*}\right]\right]\right)=-\sum_{i=1}^{2 s}\left(\left[\left[z_{i}^{*}, e\right], z_{i}\right]+\left[e,\left[z_{i}^{*}, z_{i}\right]\right]\right),
$$

we have $\sum_{i=1}^{2 s}\left[z_{i},\left[e, z_{i}^{*}\right]\right]=-s h$. As $\widehat{\rho}_{\chi}(f) 1_{\chi}=(e, f) 1_{\chi}=1_{\chi}$, it follows that

$$
\begin{aligned}
C\left(1_{\chi}\right) & =\left(2 e+\frac{h^{2}}{2}-h+\sum_{i} a_{i} b_{i}+2 \sum_{i=1}^{2 s}\left[e, z_{i}^{*}\right] z_{i}+\sum_{i=1}^{2 s}\left[z_{i},\left[e, z_{i}^{*}\right]\right]\right) \otimes 1_{\chi} \\
& =\left(2 e+\frac{h^{2}}{2}-(s+1) h+\sum_{i} a_{i} b_{i}+2 \sum_{i=1}^{2 s}\left[e, z_{i}^{*}\right] z_{i}\right) \otimes 1_{\chi} .
\end{aligned}
$$

As a consequence,

$$
\widetilde{\mu}(C)=\left(2 e+\frac{h^{2}}{2}-(s+1) h+\sum_{i} a_{i} b_{i}\right) \otimes 1+2 \sum_{i=1}^{2 s}\left[e, z_{i}^{*}\right] \otimes z_{i} .
$$

In view of 4.3.1] and the convention of 4.1 this yields

$$
\begin{aligned}
C-\Theta_{\text {Cas }}= & \left(2 e+\frac{h^{2}-(s+2) h}{2}\right) \otimes 1 \\
& +2 \sum_{i=1}^{2 s}\left[e, z_{i}^{*}\right] \otimes z_{i}+\sum_{i, j=1}^{2 s}\left[e z_{i}^{*} z_{j}^{*}\right] \otimes z_{j} z_{i}-1 \otimes c_{0}^{\prime} .
\end{aligned}
$$


Proposition 4.1. Let $u, v \in \mathfrak{z} \chi(1)$. Then the following relation holds in $H$ :

$$
\left[\Theta_{u}, \Theta_{v}\right]=\frac{1}{2}(f,[u, v])\left(C-\Theta_{\text {Cas }}-c_{0}\right)+\frac{1}{2} \sum_{i=1}^{2 s}\left(\Theta_{\left[u, z_{i}\right]^{\sharp}} \Theta_{\left[v, z_{i}^{*}\right]^{\sharp}}+\Theta_{\left[v, z_{i}^{*}\right]^{\sharp}} \Theta_{\left[u, z_{i}\right]^{\sharp}}\right),
$$

where $c_{0} \in \mathbb{k}$ is a constant depending on $\mathfrak{g}$.

Proof. Set $B(u, v):=A(u, v)-\frac{1}{2}(f,[u, v])\left(C-\Theta_{\text {Cas }}\right)$, an element in $H$. From 4.2.6 and 4.4.2) we deduce that $\tilde{\mu}(B(u, v))=1 \otimes b(u, v)$ for some $b(u, v) \in \mathbf{A}_{e}$. In conjunction with [31, Lemma 4.5] this shows that $b(u, v) \in \mathbb{k}$ for all $u, v \in \mathfrak{z} \chi(1)$. In other words, $b: \mathfrak{z}_{\chi}(1) \times \mathfrak{z}_{\chi}(1) \rightarrow \mathbb{k},(u, v) \mapsto b(u, v)$, is a bilinear form on $\mathfrak{z}_{\chi}(1)$. In view of Lemma 2.5, the $\mathfrak{z}_{\chi}(0)$-invariance of $\langle\cdot, \cdot\rangle$, and the Jacobi identity, this form is invariant under $\mathfrak{z}_{\chi}(0)$. On the other hand, it is well-known (and easily seen) that if $\mathfrak{g}$ is not of type A, then $\mathfrak{z}_{\chi}(1)$ is an irreducible $\mathfrak{z}_{\chi}(0)$-module, and if $\mathfrak{g}$ is of type A and $\mathfrak{z}_{\chi}(1) \neq 0$, then $\mathfrak{z}_{\chi}(1) \cong M \oplus M^{*}$ where $M$ is an irreducible $\mathfrak{z}_{\chi}(0)$-module such that $M \nsubseteq M^{*}$. Therefore, in all cases $b=c_{0}(f,[\cdot, \cdot])$ for some $c_{0} \in \mathbb{k}$. This completes the proof.

Let $\left\{x_{1}, \ldots, x_{n}\right\}$ and $\left\{u_{1}, \ldots, u_{2 s}\right\}$ be bases of $\mathfrak{z} \chi(0)$ and $\mathfrak{z} \chi(1)$, respectively, and let $H^{+}$ denote the $\mathbb{k}$-span of

$$
\left\{\Theta_{x_{1}}^{i_{1}} \cdots \Theta_{x_{n}}^{i_{n}} \cdot \Theta_{u_{1}}^{j_{1}} \cdots \Theta_{u_{2 s}}^{j_{2 s}} \cdot\left(C-c_{0}\right)^{l} \mid \sum i_{k}+\sum j_{k}+l \geq 1\right\},
$$

a subspace of codimension 1 in $H$; see [31, Theorem 4.6(ii)].

Corollary 4.1. The subspace $H^{+}$is a two-sided ideal of the minimal nilpotent algebra $H$. If $\mathfrak{g}$ is not of type A, then $H^{+}$is the only ideal of codimension 1 in $H$.

Proof. We need to show that $h \cdot h^{\prime} \in H^{+}$for all $h, h^{\prime} \in H^{+}$. Since $C-c_{0} \in Z(H)$, it suffices to show that $\Theta_{x} \cdot H^{+} \subset H^{+}$for all $x \in \mathfrak{z}_{\chi}(0) \cup \mathfrak{z}_{\chi}(1)$. Using Lemma 2.4 it is easy to observe that the span of all $\Theta_{x_{1}}^{i_{1}} \ldots \Theta_{x_{n}}^{i_{n}}$ with $\sum_{k=1}^{n} i_{k} \geq 1$ is stable under the left multiplications by $\Theta_{x}$ with $x \in \mathfrak{z}_{\chi}(0)$. Thus we may assume that $x \in \mathfrak{z}_{\chi}(1)$. Lemma 2.5 (in conjunction with [31, Theorem 4.6(ii)]) now provides a further reduction: it suffices to show that $\Theta_{x} \cdot\left(\Theta_{u_{j_{1}}} \cdots \Theta_{u_{j_{N}}}\right) \in H^{+}$for all $x \in \mathfrak{z} \chi(1)$ and all $j_{1}, \ldots, j_{N} \in\{1, \ldots, 2 s\}$. This follows from Proposition 4.1 and Lemma 2.5 by induction on $N$.

Suppose $\mathfrak{g}$ is not of type A. Then only one node of the extended Dynkin diagram of $\mathfrak{g}$ is linked with the node corresponding to $-\widetilde{\alpha}$. From this it is immediate that the derived subalgebra of $\mathfrak{c}_{\mathfrak{g}}\left(h_{\tilde{\alpha}}\right)$ is semisimple and has codimension 1 in $\mathfrak{c}_{\mathfrak{g}}\left(h_{\tilde{\alpha}}\right)$. Since the roots $\beta$ and $-\widetilde{\alpha}$ lie in the same $W$-orbit, the subalgebras $\mathfrak{c}_{\mathfrak{g}}\left(h_{\tilde{\alpha}}\right)$ and $\mathfrak{g}(0)=\mathfrak{c}_{\mathfrak{g}}(h)$ are conjugate under Ad $G$. It follows that $\mathfrak{z} \chi(0)=[\mathfrak{g}(0), \mathfrak{g}(0)]$ is semisimple.

Let $I$ be any ideal of codimension 1 in $H$. Then $x y-y x \in I$ for all $x, y \in H$. Since $\mathfrak{z}_{\chi}(0)$ is semisimple, we have $\mathfrak{z}_{\chi}(0)=\left[\mathfrak{z}_{\chi}(0), \mathfrak{z}_{\chi}(0)\right]$. In view of Lemma 2.4 this implies that $\Theta_{x} \in I$ for all $x \in \mathfrak{z}_{\chi}(0)$. We have already mentioned that in the present case $\mathfrak{z}_{\chi}(1)$ is an irreducible $\mathfrak{z}_{\chi}(0)$-module. So $\mathfrak{z}_{\chi}(1)=\left[\mathfrak{z}_{\chi}(0), \mathfrak{z}_{\chi}(1)\right]$, yielding $\Theta_{u} \in I$ for all $u \in \mathfrak{z}_{\chi}(1)$; see Lemma 2.5. Since $I$ is a subalgebra of $H$ containing $\mathfrak{z}_{\chi}(0) \cup$ $\mathfrak{z}_{\chi}(1)$, Proposition 4.1 entails $C-c_{0} \in I$. As a consequence, $H^{+} \subseteq I$. Since $H_{\chi}^{+}$has codimension 1 in $H$, we conclude that $I=H^{+}$, as desired. 


\section{Primitive ideals and Goldie rank}

5.1. We retain the assumptions of the previous section and denote by $\mathcal{C}$ the category $\mathcal{C}_{\chi}$ for $\chi=\left(e_{\beta}, \cdot\right)$. According to (3.1), given $M \in \mathcal{C}$ one has $M \cong Q_{\chi} \otimes_{H} M_{0}$ where $M_{0}=\mathrm{Wh}(M)$. Let $\left\{m_{i} \mid i \in I\right\}$ be a basis of $M_{0}$. It is immediate from [31, p. 52] that the vectors

$$
\left\{h^{l} z_{1}^{i_{1}} \cdots z_{s}^{i_{s}} \otimes m_{j} \mid j \in I ; l, i_{i}, \ldots, i_{s} \in \mathbb{Z}_{+}\right\}
$$

form a basis of $Q_{\chi} \otimes_{H} M_{0}$ over $\mathbb{k}$. We can thus identify $M$ and $\mathbb{k}\left[h, z_{1}, \ldots, z_{s}\right] \otimes M_{0}$ as vector spaces over $\mathbb{k}$. Recall that $\left[z_{i}, z_{j}\right]=0$ and $\left[h, z_{i}\right]=-z_{i}$ for all $i, j \leq s$.

Let $\Delta$ denote the automorphism of the polynomial algebra $\mathbb{k}[h]$ such that $\Delta(h)=$ $h+1$. Clearly, $\Delta^{-1}(h)=h-1$. Let $\langle\Delta\rangle$ stand for the cyclic subgroup of Aut $(\mathbb{k}[h])$ generated by $\Delta$. The skew group algebra $\mathbb{k}[h] *\langle\Delta\rangle$ has the set $\left\{h^{i} \Delta^{j} \mid i \in \mathbb{Z}_{+}, j \in \mathbb{Z}\right\}$ as a $\mathbb{k}$-basis and the following relations hold:

$$
\Delta^{i} \cdot h^{k}=(h+i)^{k} \cdot \Delta^{i} \quad\left(i \in \mathbb{Z}, k \in \mathbb{Z}_{+}\right) .
$$

Let $\mathcal{A}_{e}:=(\mathbb{k}[h] *\langle\Delta\rangle) \otimes \mathbf{A}_{e}$, an associative algebra over $\mathbb{k}$, and identify the Weyl algebra $\mathbf{A}_{e}$ and the skew group algebra $\mathbb{k}[h] *\langle\Delta\rangle$ with the subalgebras $\mathbb{k} \otimes \mathbf{A}_{e}$ and $(\mathbb{k}[h] *\langle\Delta\rangle) \otimes \mathbb{k}$ of $\mathcal{A}_{e}$, respectively. Define an involution $\tau \in \operatorname{Aut}\left(\mathcal{A}_{e}\right)$ by setting

$\tau\left(z_{i}\right)=-z_{i}, \quad \tau\left(\partial_{i}\right)=-\partial_{i}, \quad \tau(h)=h, \quad \tau\left(\Delta^{k}\right)=(-1)^{k} \Delta^{k} \quad(1 \leq i \leq s, k \in \mathbb{Z})$.

The polynomial algebra $\mathbb{k}\left[h, z_{1}, \ldots, z_{s}\right]=\mathbb{k}[h]\left[z_{1}, \ldots, z_{s}\right]$ has a natural $\mathcal{A}_{e}$-module structure such that $h . f\left(h, z_{1}, \ldots, z_{s}\right)=h f\left(h, z_{1}, \ldots, z_{s}\right)$ and $\Delta^{k} . f\left(h, z_{1}, \ldots, z_{s}\right)=$ $f\left(h+k, z_{1}, \ldots, z_{s}\right)$. As this $\mathcal{A}_{e}$-module is faithful, we will identify $\mathcal{A}_{e}$ with a subalgebra of $\operatorname{End}\left(\mathbb{k}\left[h, z_{1}, \ldots, z_{s}\right]\right)$. Since $\Delta^{2 k} \in \mathcal{A}_{e}^{\tau}$ for all $k \in \mathbb{Z}$ and $\Delta \otimes z_{i}, \Delta \otimes \partial_{i} \in \mathcal{A}_{e}^{\tau}$ for all $i \leq s$, it is easy to see that $\mathbb{k}\left[h, z_{1}, \ldots, z_{s}\right]$ remains irreducible when restricted to the fixed point algebra $\mathcal{A}_{e}^{\tau}$.

Let $I$ be any two-sided ideal of $H$. Since $I$ is $\sigma$-stable by Corollary 2.1 the ideal $\mathcal{A}_{e} \otimes I$ of the algebra $\mathcal{A}_{e} \otimes H$ is invariant under the involution $\tau \otimes \sigma$ of $\mathcal{A}_{e} \otimes H$. Hence $\tau \otimes \sigma$ indices an automorphism of order two on the algebra $\mathcal{A}_{e} \otimes(H / I)$. We mention for further references that $\left(\mathcal{A}_{e} \otimes(H / I)\right)^{\tau \otimes \sigma}$ contains the images in $\mathcal{A}_{e} \otimes(H / I)$ of $\mathcal{A}_{e}^{\tau} \otimes \mathbb{k}$, $1 \otimes \Theta_{a}$, and $\Delta^{-1} \otimes \Theta_{u}$ for all $a \in \mathfrak{z}_{\chi}(0)$ and $u \in \mathfrak{z}_{\chi}(1)$.

5.2. Recall that in our present setting the element $f$ is a root vector for $\mathfrak{h}$ corresponding to $-\beta$. Since ad $f$ is locally nilpotent on $U(\mathfrak{g})$, the set $S_{f}:=\left\{f^{i} \mid i \in \mathbb{Z}_{+}\right\}$is an Ore set in $U(\mathfrak{g})$; see $[13,(11.2)]$. We denote by $U(\mathfrak{g})_{f}$ the localization $S_{f}^{-1} U(\mathfrak{g})$. Since $f$ commutes with the $z_{i}$ 's and $f h^{m}=(h+2)^{m} f$ for all $m \in \mathbb{Z}_{+}$, it follows that $f$ acts on $M=\mathbb{k}\left[h, z_{1}, \ldots, z_{s}\right] \otimes M_{0}$ as $\Delta^{2} \otimes \operatorname{id}_{M_{0}}$ (one should keep in mind that $\chi(f)=1$ ). In particular, $f$ acts invertibly on $M$. From this it follows that the localization $S_{f}^{-1} M$ can be identified with $M$; see [13,(11.4)]. As a result, the action of $U(\mathfrak{g})$ on $M$ extends uniquely to a representation of $U(\mathfrak{g})_{f}$ in End $M$. We note for completeness that the enveloping algebra $U(\mathfrak{g})$ is canonically identified with a subalgebra of $U(\mathfrak{g})_{f}$. 
Theorem 5.1. Let $M \in \mathcal{C}$ and identify $M$ with $\mathbb{k}\left[h, z_{1}, \ldots, z_{s}\right] \otimes M_{0}$ where $M_{0}=$ $\mathrm{Wh}(M)$. Let $\tilde{\rho}: U(\mathfrak{g})_{f} \rightarrow$ End $M$ and $\rho: H \rightarrow$ End $M_{0}$ be the representations of $U(\mathfrak{g})_{f}$ and $H$ induced by the action of $\mathfrak{g}$ on $M$. Then the following hold:

(i) $\left(\Delta \otimes \operatorname{id}_{M_{0}}\right) \circ \tilde{\rho}\left(U(\mathfrak{g})_{f}\right) \circ\left(\Delta \otimes \operatorname{id}_{M_{0}}\right)^{-1}=\tilde{\rho}\left(U(\mathfrak{g})_{f}\right)$;

(ii) $\tilde{\rho}(f)=\Delta^{2} \otimes \operatorname{id}_{M_{0}}$;

(iii) $\mathcal{A}_{e} \otimes \rho(H)=\tilde{\rho}\left(U(\mathfrak{g})_{f}\right) \oplus \tilde{\rho}\left(U(\mathfrak{g})_{f}\right)\left(\Delta \otimes \operatorname{id}_{M_{0}}\right)$;

(iv) $\tilde{\rho}\left(U(\mathfrak{g})_{f}\right)=\left(\mathcal{A}_{e} \otimes \rho(H)\right)^{\tau \otimes \sigma}$.

Proof. Put id $=\operatorname{id}_{M_{0}}$. We have already mentioned that $\tilde{\rho}(f)=\Delta^{2} \otimes \mathrm{id}$, showing (ii). Since $z_{i} h^{m}=(h+1)^{m} z_{i}$ for all $m \in \mathbb{Z}_{+}$and $i \leq 2 s$, it follows that

$$
\tilde{\rho}(h)=h \otimes \mathrm{id}, \quad \tilde{\rho}\left(z_{i}\right)=\left(\Delta \circ z_{i}\right) \otimes \mathrm{id}, \quad \tilde{\rho}\left(z_{i+s}\right)=\left(\Delta \circ \partial_{i}\right) \otimes \mathrm{id} \quad(1 \leq i \leq s) .
$$

All these are in $\mathcal{A}_{e}^{\tau} \otimes \mathrm{id} \subset\left(\mathcal{A}_{e} \otimes \rho(H)\right)^{\tau \otimes \sigma}$. Now let $a \in \mathfrak{z}_{\chi}(0)$ and write $\left[a, z_{i}\right]=$ $\sum_{i=1}^{2 s} \mu_{i j} z_{j}$ for $1 \leq i \leq 2 s$, where $\mu_{i j} \in \mathbb{k}$. Note that for $1 \leq i, j \leq s$ we have $\left[a z_{i} z_{j}\right]=\mu_{i, j+s} f$, and

$$
\begin{aligned}
a z_{1}^{k_{1}} \cdots z_{s}^{k_{s}}= & \sum_{i=1}^{s} k_{i} z_{1}^{k_{1}} \cdots z_{i}^{k_{i}-1} \cdots z_{s}^{k_{s}}\left[a, z_{i}\right]+\frac{k_{i}\left(k_{i}-1\right)}{2} \sum_{i=1}^{s} z_{1}^{k_{1}} \cdots z_{i}^{k_{i}-2} \cdots z_{s}^{k_{s}}\left[a z_{i}^{2}\right] \\
& +k_{i} k_{j} \sum_{1 \leq i<j \leq s} z_{1}^{k_{1}} \cdots z_{i}^{k_{i}-1} \cdots z_{j}^{k_{j}-1} \cdots z_{s}^{k_{s}}\left[a z_{i} z_{j}\right]+z_{1}^{k_{1}} \cdots z_{s}^{k_{s}} a
\end{aligned}
$$

for all $k_{i} \in \mathbb{Z}_{+}$. Since $\langle\cdot, \cdot\rangle$ is $\mathfrak{z} \chi(0)$-invariant, it must be that $\mu_{i+s, j+s}=-\mu_{j i}, \mu_{i, j+s}=$ $\mu_{j, i+s}$, and $\mu_{i+s, j}=\mu_{j+s, i}$ where $1 \leq i, j \leq s$. In view of Lemma 2.3 and the fact that $z_{i+s} \in \mathfrak{m}_{\chi}$ for all $i \leq s$, we must have $\tilde{\rho}(a)(1 \otimes m)=1 \otimes \rho\left(\Theta_{a}\right)(m)-$ $\frac{1}{2} \sum_{i=1}^{s}\left[a, z_{i+s}\right] z_{i} \otimes 1$ for all $m \in M_{0}$. In conjunction with our earlier remarks (and the fact that $[a, h]=0)$ this yields

$$
\begin{aligned}
\tilde{\rho}(a)= & 1 \otimes\left(\rho\left(\Theta_{a}\right)+\sum_{i=1}^{s} \mu_{i i}\right)+\left(\sum_{i, j=1}^{s} \mu_{i j} z_{j} \partial_{i}\right) \otimes \mathrm{id} \\
& +\left(\sum_{i=1}^{s} \frac{\mu_{i, i+s}}{2} \partial_{i}^{2}+\sum_{1 \leq i<j \leq s} \mu_{i, j+s} \partial_{i} \partial_{j}\right) \otimes \mathrm{id} \\
& -\left(\sum_{i=1}^{s} \frac{\mu_{i, i+s}}{2} z_{i}^{2}+\sum_{1 \leq i<j \leq s} \mu_{i, j+s} z_{i} z_{j}\right) \otimes \mathrm{id} .
\end{aligned}
$$

This shows that $\tilde{\rho}(a)$ commutes with $\Delta \otimes$ id and lies in $\left(\mathcal{A}_{e} \otimes \rho(H)\right)^{\tau \otimes \sigma}$. Since all operators $\left(\Delta^{2} \circ z_{i} z_{j}\right) \otimes \mathrm{id},\left(\Delta^{2} \circ \partial_{i} \partial_{j}\right) \otimes \mathrm{id}$, and $\left(\Delta^{2} \circ z_{j} \partial_{i}\right) \otimes$ id are in $\tilde{\rho}\left(U\left(\mathfrak{n}_{\chi}\right)\right)$ by our remarks earlier in the proof, and since $\tilde{\rho}\left(f^{-1}\right)=\Delta^{-2} \otimes \mathrm{id}$, it also follows that $1 \otimes \rho\left(\Theta_{a}\right)$ is in $\tilde{\rho}\left(U(\mathfrak{g})_{f}\right)$ for all $a \in \mathfrak{z}_{\chi}(0)$. 
Now let $u \in \mathfrak{z}_{\chi}(1)$. First note that $\left[u, z_{i}\right]=\left[u, z_{i}\right]^{\sharp}+\frac{1}{2}\left(u, z_{i}\right) h$ and $u h^{m}=(h-1)^{m} u$ for all $i \leq s$ and $m \in \mathbb{Z}_{+}$. Next observe that

$$
\begin{aligned}
u z_{1}^{k_{1}} \cdots z_{s}^{k_{s}}= & \sum_{i=1}^{s} z_{1}^{k_{1}} \cdots z_{i}^{k_{i}-1} \cdots z_{s}^{k_{s}}\left[u, z_{i}\right]+\sum_{i=1}^{s} \frac{k_{i}\left(k_{i}-1\right)}{2} z_{1}^{k_{1}} \cdots z_{i}^{k_{i}-2} \cdots z_{s}^{k_{s}}\left[u z_{i}^{2}\right] \\
& +\sum_{1 \leq i<j \leq s} k_{i} k_{j} z_{1}^{k_{1}} \cdots z_{i}^{k_{i}-1} \cdots z_{j}^{k_{j}-1} \cdots z_{s}^{k_{s}}\left[u z_{i} z_{j}\right] \\
& +\sum_{1 \leq i<j<l \leq s} k_{i} k_{j} k_{l} z_{1}^{k_{1}} \cdots z_{i}^{k_{i}-1} \cdots z_{j}^{k_{j}-1} \cdots z_{l}^{k_{l}-1} \cdots z_{s}^{k_{s}}\left[u z_{i} z_{j} z_{l}\right] \\
& +\sum_{1 \leq i<j \leq s} \frac{k_{i}\left(k_{i}-1\right)}{2} k_{j} z_{1}^{k_{1}} \cdots z_{i}^{k_{i}-2} \cdots z_{j}^{k_{j}-1} \cdots z_{s}^{k_{s}}\left[u z_{i}^{2} z_{j}\right] \\
& +\sum_{1 \leq i<j \leq s} k_{i} \frac{k_{j}\left(k_{j}-1\right)}{2} z_{1}^{k_{1}} \cdots z_{i}^{k_{i}-1} \cdots z_{j}^{k_{j}-2} \cdots z_{s}^{k_{s}}\left[u z_{i} z_{j}^{2}\right] \\
& +\sum_{i=1}^{s} \frac{k_{i}\left(k_{i}-1\right)\left(k_{i}-2\right)}{6} z_{1}^{k_{1}} \cdots z_{i}^{k_{i}-3} \cdots z_{s}^{k_{s}}\left[u z_{i}^{3}\right]+z_{1}^{k_{1}} \cdots z_{s}^{k_{s}} u
\end{aligned}
$$

and

$$
\begin{aligned}
z_{1}^{k_{1}} \cdots z_{s}^{k_{s}} h & =h z_{1}^{k_{1}} \cdots z_{s}^{k_{s}}-\left[h, z_{1}^{k_{1}} \cdots z_{s}^{k_{s}}\right]=h z_{1}^{k_{1}} \cdots z_{s}^{k_{s}}+\left(k_{1}+\cdots+k_{s}\right) z_{1}^{k_{1}} \cdots z_{s}^{k_{s}} \\
& =\left(h+\sum_{i=1}^{s} z_{i} \partial_{i}\right)\left(z_{1}^{k_{1}} \cdots z_{s}^{k_{s}}\right) .
\end{aligned}
$$

This shows that for all $m \in M_{0}$ we have

$$
\begin{aligned}
& \sum_{i=1}^{s} z_{1}^{k_{1}} \cdots z_{i}^{k_{i}-1} \cdots z_{s}^{k_{s}} \tilde{\rho}\left(\left[u, z_{i}\right]\right)(1 \otimes m) \\
& =\left(\left(h+\sum_{i=1}^{s} z_{i} \partial_{i}\right) \circ\left(\sum_{i=1}^{s} \frac{\left(u, z_{i}\right)}{2} \partial_{i}\right)+\sum_{i=1}^{s} \partial_{i} \otimes \rho\left(\Theta_{\left[u, z_{i}\right]^{\sharp}}\right)\right)\left(z_{1}^{k_{1}} \cdots z_{s}^{k_{s}} \otimes m\right) \\
& \quad-\frac{1}{2} \sum_{i, j=1}^{s} z_{1}^{k_{1}} \cdots z_{i}^{k_{i}-1} \cdots z_{s}^{k_{s}} \tilde{\rho}\left(\left[\left[u, z_{i}\right]^{\sharp}, z_{j+s}\right] z_{j}\right)(1 \otimes m) .
\end{aligned}
$$

In view of Lemma 2.5 we now get

$$
\begin{aligned}
& z_{1}^{k_{1}} \cdots z_{s}^{k_{s}} \tilde{\rho}(u)(1 \otimes m)=\left(\mathrm{id} \otimes \rho\left(\Theta_{u}\right)\right)\left(z_{1}^{k_{1}} \cdots z_{s}^{k_{s}} \otimes m\right) \\
& -z_{1}^{k_{1}} \cdots z_{s}^{k_{s}}\left(\sum_{i=1}^{s} z_{i} \tilde{\rho}\left(\left[u, z_{i+s}\right]\right)(1 \otimes m)+\frac{1}{3} \sum_{i, j=1}^{s} \tilde{\rho}\left(\left[u z_{i+s} z_{j+s}\right]\right)\left(z_{j} z_{i} \otimes m\right)\right. \\
& \left.+\frac{2}{3} \sum_{i=1}^{s} \tilde{\rho}\left(\left[u z_{i+s} z_{i}\right]\right)(1 \otimes m)+\tilde{\rho}\left(z_{u}\right)(1 \otimes m)\right)
\end{aligned}
$$


Together with the above remarks this says that $\tilde{\rho}(u)$ is a linear combination of the following operators:

$$
\begin{aligned}
& \left(h+\sum_{i=1}^{s} z_{i} \partial_{i}\right) \circ\left(\sum_{i=1}^{s} \frac{\left(u, z_{i}\right)}{2} \partial_{i}\right) \circ \Delta^{-1} \otimes \mathrm{id}, \sum_{i=1}^{s}\left(\partial_{i} \circ \Delta^{-1}\right) \otimes \rho\left(\Theta_{\left[u, z_{i}\right]^{\sharp}}\right), \\
& \left(h+\sum_{i=1}^{s} z_{i} \partial_{i}\right) \circ\left(\sum_{i=1}^{s} \frac{\left(u, z_{i+s}\right)}{2} z_{i}\right) \circ \Delta^{-1} \otimes \mathrm{id}, \sum_{i=1}^{s}\left(z_{i} \circ \Delta^{-1}\right) \otimes \rho\left(\Theta_{\left[u, z_{i+s}\right]^{\sharp}}\right), \\
& \left(\partial_{i} \partial_{j} \partial_{k} \circ \Delta^{-1}\right) \otimes \mathrm{id},\left(z_{i} z_{j} z_{k} \circ \Delta^{-1}\right) \otimes \mathrm{id},\left(z_{i} z_{j} \partial_{k} \circ \Delta^{-1}\right) \otimes \mathrm{id}, \\
& \left(z_{k} \partial_{i} \partial_{j} \circ \Delta^{-1}\right) \otimes \mathrm{id},\left(\partial_{i} \circ \Delta^{-1}\right) \otimes \mathrm{id},\left(z_{i} \circ \Delta^{-1}\right) \otimes \mathrm{id}, \Delta^{-1} \otimes \rho\left(\Theta_{u}\right),
\end{aligned}
$$

where $i, j, k \leq s$. But then $\tilde{\rho}(u) \in\left(\mathcal{A}_{e} \otimes \rho(H)\right)^{\sigma \otimes \tau}$ and $(\Delta \otimes \mathrm{id}) \tilde{\rho}(u)(\Delta \otimes \mathrm{id})^{-1}-\tilde{\rho}(u) \in$ $\mathcal{A}_{e}^{\tau} \otimes$ id. As $\mathcal{A}_{e}^{\tau} \otimes \mathrm{id} \subset \tilde{\rho}\left(U(\mathfrak{g})_{f}\right)$ by our earlier remarks, this yields

$$
(\Delta \otimes \mathrm{id}) \tilde{\rho}(x)(\Delta \otimes \mathrm{id})^{-1} \in \tilde{\rho}\left(U(\mathfrak{g})_{f}\right) \quad\left(\forall x \in \bigcup_{i \leq 1} \mathfrak{g}(i)\right) .
$$

Since $\mathfrak{g}(2)=[\mathfrak{g}(1), \mathfrak{g}(1)]$, we obtain (i) and the inclusion $\tilde{\rho}\left(U(\mathfrak{g})_{f}\right) \subseteq\left(\mathcal{A}_{e} \otimes \rho(H)\right)^{\sigma \otimes \tau}$. Notice that $\Delta^{-1} \otimes \rho\left(\Theta_{u}\right) \in \mathcal{A}_{e}^{\tau} \otimes \mathrm{id}+\tilde{\rho}\left(U(\mathfrak{g})_{f}\right)$ for all $u \in \mathfrak{z}_{\chi}(1)$ and $1 \otimes \rho\left(\Theta_{a}\right) \in$ $\mathcal{A}_{e}^{\tau} \otimes \mathrm{id}+\tilde{\rho}\left(U(\mathfrak{g})_{f}\right)$ for all $a \in \mathfrak{z}_{\chi}(0)$; see $(5.2 .1)$. Since the algebra $H$ is generated by the elements $\Theta_{x}$ with $x \in \mathfrak{z}_{\chi}(0) \cup \mathfrak{z}_{\chi}(1)$, by Proposition 4.1 , we get

$$
1 \otimes \rho(H) \subset \tilde{\rho}\left(U(\mathfrak{g})_{f}\right)+\tilde{\rho}\left(U(\mathfrak{g})_{f}\right)(\Delta \otimes \mathrm{id}) .
$$

Since $\mathcal{A}_{e}=\mathcal{A}_{e}^{\tau}+\mathcal{A}_{e}^{\tau} \Delta$ and $\tilde{\rho}\left(U(\mathfrak{g})_{f}\right) \subseteq\left(\mathcal{A}_{e} \otimes \rho(H)\right)^{\sigma \otimes \tau}$ we derive (iii). Then (iv) follows, completing the proof.

5.3. By [31, (6.2)], the restriction of the representation $\tilde{\rho}_{\chi}: U(\mathfrak{g}) \rightarrow \operatorname{End}\left(Q_{\chi}\right)$ to the centre $Z(\mathfrak{g})$ of $U(\mathfrak{g})$ is injective. Since any nonzero two-sided ideal of $U(\mathfrak{g})$ intersects $Z(\mathfrak{g})$ by $[9,(4.2 .2)]$, it follows that $\tilde{\rho}_{\chi}$ is a faithful representation of $U(\mathfrak{g})$. Note that $Q_{\chi} \in \mathcal{C}$ and $\operatorname{Wh}\left(Q_{\chi}\right)$ is canonically identified with $H$ (viewed as the left regular $H$ module). By 5.1. $Q_{\chi} \cong Q_{\chi} \otimes_{H} H$ is then identified with $\mathcal{A}_{e} \otimes H$ as vector spaces over $\mathbb{k}$. Since $\tilde{\rho}$ is faithful, it extends to a faithful representation of $U(\mathfrak{g})_{f}$ in $\operatorname{End}\left(Q_{\chi}\right)$ (one should take into account that $\tilde{\rho}_{\chi}(f)$ is invertible). Since $\operatorname{Wh}\left(Q_{\chi}\right)$ is identified with the left regular $H$-module, Theorem 5.1 iv) yields $\tilde{\rho}_{\chi}\left(U(\mathfrak{g})_{f}\right) \subseteq \mathcal{A}_{e} \otimes H$. Applying Theorem 5.1 in this situation we now obtain:

Corollary 5.1. Set $\widehat{\Delta}=\Delta \otimes \mathrm{id}_{H}$ and identify $U(\mathfrak{g})_{f}$ with its image in $\mathcal{A}_{e} \otimes H$. Then the following hold:

(i) $\widehat{\Delta} U(\mathfrak{g})_{f} \widehat{\Delta}^{-1}=U(\mathfrak{g})_{f}$;

(ii) $f=\widehat{\Delta}^{2}$;

(iii) $\mathcal{A}_{e} \otimes H=U(\mathfrak{g})_{f} \oplus U(\mathfrak{g})_{f} \widehat{\Delta}$;

(iv) $U(\mathfrak{g})_{f}=\left(\mathcal{A}_{e} \otimes H\right)^{\tau \otimes \sigma}$.

(v) $\left(\mathcal{A}_{e} \otimes H\right)^{\mathfrak{n}_{x}}=\mathbb{k}\left[\Delta, \Delta^{-1}\right] \otimes H=\left(U(\mathfrak{g})^{\mathfrak{n}_{x}}\right)_{f} \oplus\left(U(\mathfrak{g})^{\mathfrak{n}_{x}}\right)_{f} \widehat{\Delta}$;

(vi) $Z(H) \cong Z\left(U(\mathfrak{g})_{f}\right) \cong Z(\mathfrak{g})$. 
Proof. Parts (i)-(iv) follow from Theorem 5.1 and the discussion above. For (v), note that $\left(\Delta \circ z_{i}\right) \otimes 1,\left(\Delta \circ \partial_{i}\right) \otimes 1 \in \mathfrak{n}_{\chi}$ for all $i \leq s$ (see the beginning of the proof of Theorem 5.1). From this it is immediate that $\left(\mathcal{A}_{e} \otimes H\right)^{\mathfrak{n}_{x}}=\mathbb{k}\left[\Delta, \Delta^{-1}\right] \otimes H$. Since $f \in Z\left(U(\mathfrak{g})^{\mathfrak{n}_{x}}\right)$ we have $\left(U(\mathfrak{g})_{f}\right)^{\mathfrak{n}_{\chi}}=\left(U(\mathfrak{g})^{\mathfrak{n}_{\chi}}\right)_{f}$, hence the result.

For (vi), we first recall that the action of $1 \otimes \sigma$ on $\mathcal{A}_{e} \otimes H$ is induced by the adjoint action of $1 \otimes \Theta\left(\mathfrak{z}_{\chi}(0)\right) \subset 1 \otimes H$. Consequently,

$$
Z(H) \cong Z\left(\mathcal{A}_{e} \otimes H\right)=\mathbb{k} \otimes\left(H^{\sigma} \cap Z(H)\right)=\left(Z\left(\mathcal{A}_{e} \otimes H\right)\right)^{\tau \otimes \sigma} \subseteq Z\left(U(\mathfrak{g})_{f}\right),
$$

by (iv). On the other hand, $Z\left(U(\mathfrak{g})_{f}\right) \subseteq Z\left(\left(U(\mathfrak{g})^{\mathfrak{n}_{\chi}}\right)_{f}\right) \subseteq \mathbb{k}\left[\Delta, \Delta^{-1}\right] \otimes Z(H)$, by (v). Since $[h, \Delta]=-\Delta$, it must be that $Z\left(U(\mathfrak{g})_{f}\right) \subseteq \mathbb{k} \otimes Z(H)=Z\left(\mathcal{A}_{e} \otimes H\right)$. Therefore, $Z(H) \cong Z\left(U(\mathfrak{g})_{f}\right)$.

It remains to show that $Z\left(U(\mathfrak{g})_{f}\right)=Z(\mathfrak{g})$. This is easy and must be well-known, but we could not find a good reference. One can argue as follows: Let $K(\mathfrak{g})=\operatorname{Fract} U(\mathfrak{g})$ be the Lie field of $\mathfrak{g}$. By $[9,(4.3 .2)]$, the centre of $K(\mathfrak{g})$ coincides with Fract $Z(\mathfrak{g})$. Let $z \in Z\left(U(\mathfrak{g})_{f}\right)$. Then $z=f^{-d} c$ for some $c \in U(\mathfrak{g})$ and $d \in \mathbb{Z}_{+}$. Since $Z\left(U(\mathfrak{g})_{f}\right) \subset$ $Z(K(\mathfrak{g}))$, there are $a, b \in Z(\mathfrak{g})$ such that $a f^{d}=b c$. By a classical result of Kostant, $U(\mathfrak{g}) \cong Z(\mathfrak{g}) \otimes \mathrm{H}(\mathfrak{g})$ as $Z(\mathfrak{g})$-modules, where $\mathrm{H}(\mathfrak{g})$ denotes the subspace of $U(\mathfrak{g})$ spanned by the powers of the nilpotent elements of $\mathfrak{g}$; see [9, (8.2.4) and (8.5.5)]. Choose a basis $\left\{u_{i}\right\}$ in $\mathrm{H}(\mathfrak{g})$ with $u_{1}=f^{d}$. Write $c=\sum_{i} z_{i} u_{i}$ with $z_{i} \in Z(\mathfrak{g})$. Then $a=b z_{1}$ and $z_{i}=0$ for $i \neq 1$, forcing $z=f^{-d} c=f^{-d} z_{1} f^{d}=z_{1} \in Z(\mathfrak{g})$. The result follows.

5.4. Recall that a two-sided ideal $I$ of an associative ring $R$ is called prime if $I \neq R$ and for any two two-sided ideals $J_{1}, J_{2}$ the inclusion $J_{1} J_{2} \subseteq I$ implies that either $J_{1} \subseteq I$ or $J_{2} \subseteq I$. We let Spec $R$ denote the set of all prime ideals of $R$. Any primitive ideal of $R$ is prime. The ring $R$ is termed prime if $(0) \in \operatorname{Spec} R$.

According to [9, (3.6.17)], if $R$ is Noetherian and $S$ is an Ore set in $R$, then the mapping $I \mapsto S^{-1} I$ sets up a bijection between the subset $(\operatorname{Spec} R)_{S}:=\{I \in \operatorname{Spec} R \mid I \cap S$ $=\emptyset\}$ of $\operatorname{Spec} R$ and $\operatorname{Spec} S^{-1} R$. On the other hand, the complement to $(\operatorname{Spec} U(\mathfrak{g}))_{S_{f}}$ $=\left\{I \in \operatorname{Spec} U(\mathfrak{g}) \mid f^{n} \notin I\right.$ for all $\left.n \in \mathbb{Z}_{+}\right\}$in Spec $U(\mathfrak{g})$ consists of all prime ideals of finite codimension in $U(\mathfrak{g})$; see [13, Lemma 13.17] for example. Thus the mapping $I \mapsto S_{f}^{-1} I$ is a bijection between the set of all prime ideals of infinite codimension in $U(\mathfrak{g})$ and the set of all prime ideals of $U(\mathfrak{g})_{f}$.

By Goldie's theorem, the set $S$ of all regular elements of a prime Noetherian ring $R$ is an Ore set in $R$ and the localisation $\mathcal{Q}(R):=S^{-1} R$ is isomorphic to the matrix algebra $\operatorname{Mat}_{n}(K)$ over a noncommutative field $K$. Both $K$ and $n$ can be described intrinsically, hence are uniquely determined by $R$. They are called the Goldie field and the Goldie rank of $R$, respectively. We write $n=\operatorname{rk}(R)$.

5.5. Corollary 5.1 (iv) allows us to identify $U(\mathfrak{g})_{f}$ with the subalgebra $\left(\mathcal{A}_{e} \otimes H\right)^{\tau \otimes \sigma}$ of $\mathcal{A}_{e} \otimes H$. Since $\mathcal{A}_{e}=\mathcal{A}_{e}^{\tau} \oplus \mathcal{A}_{e}^{\tau} \Delta$ and $\tau(\Delta)=-\Delta$, we obtain the decomposition

$$
U(\mathfrak{g})_{f}=\mathcal{A}_{e}^{\tau} \otimes H_{+} \oplus \mathcal{A}_{e}^{\tau} \Delta \otimes H_{-}
$$

where $H_{+}=\{x \in H \mid \sigma(x)=x\}$ and $H_{-}=\{x \in H \mid \sigma(x)=-x\}$. Let $I$ be any two-sided ideal of $H$. We let $\operatorname{Dim}(H / I)$ denote the Gelfand-Kirillov dimension of the 
factor algebra $H / I$. Since $I$ is $\sigma$-stable (Corollary 2.1), we have $I=I_{+} \oplus I_{-}$where $I_{ \pm}=I \cap H_{ \pm}$. Put

$$
\tilde{I}_{f}:=\mathcal{A}_{e}^{\tau} \otimes I_{+} \oplus \mathcal{A}_{e}^{\tau} \Delta \otimes I_{-} \quad \text { and } \quad \tilde{I}:=\widetilde{I}_{f} \cap U(\mathfrak{g}) .
$$

Clearly, $\widetilde{I}_{f}$ and $\tilde{I}$ are two-sided ideals of $U(\mathfrak{g})_{f}$ and $U(\mathfrak{g})$, respectively. Let $\mathcal{O}_{\text {min }}$ denote the minimal nilpotent orbit $\left(\mathrm{Ad}^{*} G\right) \cdot \chi$ in $\mathfrak{g}^{*}$. Recall that

$$
\operatorname{dim} \mathcal{O}_{\min }=2 \operatorname{dim} \mathfrak{m}_{\chi}=2(s+1) .
$$

Theorem 5.2. The following are true:

(i) The map $I \mapsto \widetilde{I}_{f}$ sets up a bijection between the set of all two-sided ideals of $H$ and the set of all two-sided ideals of $U(\mathfrak{g})_{f}$. For any two-sided ideal I of $H$ one has

$$
\operatorname{Dim}\left(U(\mathfrak{g})_{f} / \widetilde{I}_{f}\right)=\operatorname{Dim}(H / I)+\operatorname{dim} \mathcal{O}_{\min } .
$$

(ii) The map $I \mapsto \widetilde{I}$ is a bijection between $\operatorname{Spec} H$ and the set of all prime ideals of infinite codimension in $U(\mathfrak{g})$. Furthermore,

$$
\operatorname{Dim}(U(\mathfrak{g}) / \widetilde{I})=\operatorname{Dim}(H / I)+\operatorname{dim} \mathcal{O}_{\min } \quad(\forall I \in \operatorname{Spec} H) .
$$

Proof. (a) Let $\boldsymbol{\Xi}$ denote the set of all quadruples $(m, n, \mathbf{i}, \mathbf{j})$ with $m \in \mathbb{Z}_{+}, n \in \mathbb{Z}$, and $\mathbf{i}, \mathbf{j} \in \mathbb{Z}_{+}^{s}$. Order the elements in $\Xi$ lexicographically. Given $\xi=(m, n, \mathbf{i}, \mathbf{j}) \in \Xi$ define $a_{\xi} \in \mathcal{A}_{e}$ by setting $a_{\xi}=h^{m} \Delta^{n} z_{1}^{i_{1}} \cdots z_{s}^{j_{s}} \partial_{1}^{j_{1}} \ldots \partial_{s}^{j_{s}}$, where $\mathbf{i}=\left(i_{1}, \ldots, i_{s}\right)$ and $\mathbf{j}=$ $\left(j_{1}, \ldots, j_{s}\right)$. Any nonzero $x \in \mathcal{A}_{e} \otimes H$ can be written uniquely as $x=\sum_{\xi \in \Xi(x)} a_{\xi} \otimes h_{\xi}$ for some nonzero $h_{\xi} \in H$. Here $\Xi(x)$ is a finite subset of $\Xi$ depending on $x$.

Let $\mathcal{I}$ be any two-sided ideal of $U(\mathfrak{g})_{f}=\left(\mathcal{A}_{e} \otimes H\right)^{\tau \otimes \sigma}$. Recall that the action of $\sigma$ on $H$ is induced by the adjoint action of $\Theta\left(\mathfrak{z}_{\chi}(0)\right)$. Since $1 \otimes \Theta\left(\mathfrak{z}_{\chi}(0)\right) \subset\left(\mathcal{A}_{e} \otimes H\right)^{\tau \otimes \sigma}$, the ideal $\mathcal{I}$ is stable under the involution $1 \otimes \sigma$ of $\mathcal{A}_{e} \otimes H$. It follows that $\mathcal{I}=\mathcal{I}_{+} \oplus \mathcal{I}_{-}$ where $\mathcal{I}_{ \pm}=\{x \in \mathcal{I} \mid(1 \otimes \sigma)(x)= \pm x\}$. Let $x \in \mathcal{I}_{+} \cup \mathcal{I}_{-}$and let $\xi_{0}=\left(m_{0}, n_{0}, \mathbf{a}, \mathbf{b}\right)$ be the maximal element in $\Xi(x)$. Then there exists a polynomial $f_{x}(t) \in \mathbb{k}[t]$ such that

$$
f_{x}(\operatorname{ad} h) \circ\left(\prod_{i=1}^{s}\left(\operatorname{ad} \Delta \otimes \partial_{i}\right)^{a_{i}} \circ \prod_{i=1}^{s}\left(\operatorname{ad} \Delta \otimes z_{i}\right)^{b_{i}}\right) \circ\left(\operatorname{ad} \widehat{\Delta}^{2 m_{0}}\right)(x) \in \mathbb{k}^{\times} \Delta^{N+n_{0}} \otimes h_{\xi_{0}}
$$

where $N=2 m_{0}+\sum_{i=1}^{s}\left(a_{i}+b_{i}\right)$. As $\widehat{\Delta}^{2}$ is invertible, it follows that $\Delta^{\varepsilon(x)} \otimes h_{\xi_{0}} \in \mathcal{I}_{ \pm}$, where $\varepsilon(x)=0$ if $x \in \mathcal{I}_{+}$and $\varepsilon(x)=1$ if $x \in \mathcal{I}_{-}$. But then $a_{\xi_{0}} \Delta^{-\varepsilon(x)} \otimes 1 \in \mathcal{A}_{e}^{\tau} \otimes \mathbb{k} \subset$ $\left(\mathcal{A}_{e} \otimes H\right)^{\tau \otimes \sigma}$ yielding $a_{\xi_{0}} \otimes h_{\xi_{0}} \in \mathcal{I}_{ \pm}$. Continuing this process one eventually observes that $a_{\xi} \otimes h_{\xi} \in \mathcal{I}_{ \pm}$for all $\xi \in \Xi(x)$. This implies that there is a graded subspace $I=$ $I_{+} \oplus I_{-}$in $H=H_{+} \oplus H_{-}$such that $\mathcal{I}=\mathcal{A}_{e}^{\tau} \otimes I_{+} \oplus \mathcal{A}_{e}^{\tau} \Delta \otimes I_{-}$. Since $1 \otimes H_{+} \cup \Delta \otimes H_{-} \subset$ $\left(\mathcal{A}_{e} \otimes H\right)^{\tau \otimes \sigma}$ and $\widehat{\Delta}^{2}$ is invertible, it follows that $I$ is a two-sided ideal of $H$. As a result, $\mathcal{I}=\widetilde{I}_{f}$, showing that the map $I \mapsto \widetilde{I}_{f}$ is surjective. The injectivity of this map is obvious.

(b) Let $\Xi_{\geq 0}$ be the subset of $\Xi$ consisting of all $(m, n, \mathbf{i}, \mathbf{j})$ with $n \geq 0$, and let $\mathcal{A}_{e}^{\prime}$ denote the $\mathbb{k}$-span of all $a_{\xi}$ with $\xi \in \Xi_{\geq 0}$. Clearly, $\mathcal{A}_{e}^{\prime}$ is a $\tau$-invariant subalgebra of $\mathcal{A}_{e}$. For $l \in \mathbb{Z}_{+}$we let $\mathcal{A}_{e, l}^{\prime}$ denote the $\mathbb{k}$-span of all $a_{\xi}$ with $\xi=(m, n, \mathbf{i}, \mathbf{j}) \in \Xi_{\geq 0}$ such that 
$m+n+\sum_{k=1}^{s}\left(i_{k}+j_{k}\right) \leq l$. It is easy to see that $\left\{\mathcal{A}_{e, l}^{\prime} \mid l \in \mathbb{Z}_{+}\right\}$is an increasing $\tau$-invariant filtration in $\mathcal{A}_{e}^{\prime}$ and the corresponding graded algebra gr $\mathcal{A}_{e}^{\prime}$ is isomorphic to the graded polynomial algebra $\mathbb{k}\left[X_{1}, \ldots, X_{2 s+2}\right]$ with all $X_{i}$ having degree 1 . Note that $\operatorname{ad}\left(\Delta^{2}\right)$ is locally nilpotent on $\mathcal{A}_{e}^{\prime}$ and the algebra $\mathcal{A}_{e}$ identifies with the localisation $\left(\mathcal{A}_{e}^{\prime}\right)_{\Delta^{2}}$.

Let $I$ be a two-sided ideal of $H$. It follows from part (a) of this proof that the twosided ideal $\widetilde{I}_{f} \oplus \widetilde{I}_{f} \widehat{\Delta}$ of $\mathcal{A}_{e} \otimes H$ coincides with $\mathcal{A}_{e} \otimes I$, so that $\widetilde{I}_{f}=\left(\mathcal{A}_{e} \otimes I\right)^{\tau \otimes \sigma}$. Therefore, the involution $\tau \otimes \sigma$ acts on the algebra $\mathcal{A}_{e} \otimes(H / I) \cong\left(\mathcal{A}_{e} \otimes H\right) /\left(\mathcal{A}_{e} \otimes I\right)$ in such a way that the quotient $U(\mathfrak{g})_{f} / \widetilde{I}_{f}$ identifies with $\left(\mathcal{A}_{e} \otimes(H / I)\right)^{\tau \otimes \sigma}$. Since $f=\widehat{\Delta}^{2}$, it is straightforward to see that

$$
\begin{aligned}
\left(\mathcal{A}_{e} \otimes(H / I)\right)^{\tau \otimes \sigma} & \cong\left(\left(\mathcal{A}_{e}^{\prime}\right)_{\Delta^{2}} \otimes(H / I)\right)^{\tau \otimes \sigma} \cong\left(\left(\mathcal{A}_{e}^{\prime} \otimes(H / I)\right)_{\bar{f}}\right)^{\tau \otimes \sigma} \\
& \cong\left(\left(\mathcal{A}_{e}^{\prime} \otimes(H / I)\right)^{\tau \otimes \sigma}\right)_{\bar{f}}
\end{aligned}
$$

where $\bar{f}$ denotes the image of $f$ in $\mathcal{A}_{e} \otimes(H / I)$. In view of [3, (6.3)] we then have

$$
\operatorname{Dim}\left(U(\mathfrak{g})_{f} / \widetilde{I}_{f}\right)=\operatorname{Dim}\left(\left(\mathcal{A}_{e}^{\prime} \otimes(H / I)\right)^{\tau \otimes \sigma} ;\right.
$$

see also [13, (11A.2)]. The Kazhdan filtration $\left\{H^{k} \mid k \in \mathbb{Z}_{+}\right\}$gives rise to the natural filtration $\left\{(H / I)^{k}=H^{k} /\left(H^{k} \cap I\right) \mid k \in \mathbb{Z}_{+}\right\}$of the algebra $H / I$. Thanks to [31, Theorem 4.6(iii)], gr $H$ is a Noetherian commutative $\mathbb{k}$-algebra. Hence so is the corresponding graded algebra $\operatorname{gr}(H / I) \cong \operatorname{gr} H / \operatorname{gr} I$. Since $\sigma$ preserves both $I$ and the Kazhdan filtration of $H$, it induces an automorphism of the graded algebra $\operatorname{gr}(H / I)$.

Next we observe that the subspaces

$$
\left\{\left(\mathcal{A}_{e}^{\prime} \otimes(H / I)\right)_{k}=\sum_{i+j \leq k} \mathcal{A}_{e, i}^{\prime} \otimes(H / I)^{j} \mid k \in \mathbb{Z}_{+}\right\}
$$

form an increasing filtration of the algebra $\mathcal{A}_{e}^{\prime} \otimes(H / I)$ such that

$$
\operatorname{gr}\left(\mathcal{A}_{e}^{\prime} \otimes(H / I)\right) \cong \operatorname{gr}\left(\mathcal{A}_{e}^{\prime}\right) \otimes \operatorname{gr}(H / I) \cong \mathbb{k}\left[X_{1}, \ldots, X_{2 s+2}\right] \otimes \operatorname{gr}(H / I) .
$$

By construction, the involution $\tau \otimes \sigma$ acts on the graded algebra $\operatorname{gr}\left(\mathcal{A}_{e}^{\prime} \otimes(H / I)\right)$ and

$$
\left(\operatorname{gr}\left(\mathcal{A}_{e}^{\prime} \otimes(H / I)\right)\right)^{\tau \otimes \sigma} \cong \operatorname{gr}\left(\left(\mathcal{A}_{e}^{\prime} \otimes(H / I)\right)^{\tau \otimes \sigma}\right)
$$

as graded algebras. Since the morphism

$$
\operatorname{Spec} \operatorname{gr}\left(\mathcal{A}_{e}^{\prime} \otimes(H / I)\right) \rightarrow \operatorname{Spec}\left(\operatorname{gr}\left(\mathcal{A}_{e}^{\prime} \otimes(H / I)\right)\right)^{\tau \otimes \sigma}
$$

induced by inclusion $\left(\operatorname{gr}\left(\mathcal{A}_{e}^{\prime} \otimes(H / I)\right)\right)^{\tau \otimes \sigma} \hookrightarrow \operatorname{gr}\left(\mathcal{A}_{e}^{\prime} \otimes(H / I)\right)$ is finite, the Noetherian $\mathbb{k}$-algebras $\left(\operatorname{gr}\left(\mathcal{A}_{e}^{\prime} \otimes(H / I)\right)\right)^{\tau \otimes \sigma}$ and $\operatorname{gr}\left(\mathcal{A}_{e}^{\prime}\right) \otimes \operatorname{gr}(H / I)$ have the same Krull dimension. Since the Krull dimensions of the graded algebras $\left(\operatorname{gr}\left(\mathcal{A}_{e}^{\prime} \otimes(H / I)\right)\right)^{\tau \otimes \sigma}$ and $\operatorname{gr}(H / I)$ coincide with the degrees of their respective Hilbert polynomials, we derive that

$$
\operatorname{Dim} \operatorname{gr}\left(\left(\mathcal{A}_{e}^{\prime} \otimes(H / I)\right)^{\tau \otimes \sigma}\right)=2(s+1)+\operatorname{Dim} \operatorname{gr}(H / I) .
$$


On the other hand, it follows from [25, Proposition 8.6.6] that $\operatorname{Dim} \operatorname{gr}(H / I)=\operatorname{Dim}(H / I)$ and $\operatorname{Dim} \operatorname{gr}\left(\left(\mathcal{A}_{e}^{\prime} \otimes(H / I)\right)^{\tau \otimes \sigma}\right)=\operatorname{Dim}\left(\left(\mathcal{A}_{e}^{\prime} \otimes(H / I)\right)^{\tau \otimes \sigma}\right)$. Combining this with 5.5.2 we get (i).

(c) Let $I \in \operatorname{Spec} H$ and suppose $\mathcal{J}_{1} \mathcal{J}_{2} \subseteq \tilde{I}_{f}$ for some two-sided ideals $\mathcal{J}_{1}$ and $\mathcal{J}_{2}$ of $U(\mathfrak{g})_{f}=\left(\mathcal{A}_{e} \otimes H\right)^{\tau \otimes \sigma}$. By part (a), there exist two-sided ideals $J_{1}$ and $J_{2}$ of $H$ such that $\mathcal{J}_{i}=\mathcal{A}_{e}^{\tau} \otimes J_{i,+} \oplus \mathcal{A}_{e}^{\tau} \Delta \otimes J_{i,-}, i=1,2$. Since $\widehat{\Delta}^{2}$ is invertible, it is easy to see that $J_{1} J_{2} \subseteq I$. Then either $J_{1} \subseteq I$ or $J_{2} \subseteq I$, and hence either $\mathcal{J}_{1} \subseteq \widetilde{I}_{f}$ or $\mathcal{J}_{2} \subseteq \widetilde{I}_{f}$. As a result, $\widetilde{I}_{f} \in \operatorname{Spec} U(\mathfrak{g})_{f}$ and $\widetilde{I}=\widetilde{I}_{f} \cap \bar{U}(\mathfrak{g}) \in \operatorname{Spec} U(\mathfrak{g})$.

Conversely, let $\mathcal{I}$ be a prime ideal of infinite codimension in $U(\mathfrak{g})$. The discussion at the beginning of this subsection shows that $S_{f}^{-1} \mathcal{I} \in \operatorname{Spec} U(\mathfrak{g})_{f}$. By part (a) of this proof, there is a two-sided ideal $I$ of $H$ such that $S_{f}^{-1} \mathcal{I}=\mathcal{A}_{e}^{\tau} \otimes I_{+} \oplus \mathcal{A}_{e}^{\tau} \Delta \otimes I_{-}$. Clearly, $\mathcal{I}=\widetilde{I}_{f} \cap U(\mathfrak{g})=\widetilde{I}$. If $P Q \subseteq I$ for some two-sided ideals $P, Q$ of $H$, then $\widetilde{P}_{f} \widetilde{Q}_{f} \subseteq S_{f}^{-1} \mathcal{I}$, forcing either $\widetilde{P}_{f} \subseteq S_{f}^{-1} \mathcal{I}$ or $\widetilde{Q}_{f} \subseteq S_{f}^{-1} \mathcal{I}$. As $S_{f}^{-1} \mathcal{I}=\widetilde{I}_{f}$, we infer that either $P \subseteq I$ or $Q \subseteq I$. Therefore, $I \in \operatorname{Spec} H$.

Now let $\bar{f}$ be the image of $f$ in $U(\mathfrak{g}) / \widetilde{I}$ where $I \in$ Spec $H$. Since $U(\mathfrak{g})_{f} / \widetilde{I}_{f} \cong$ $(U(\mathfrak{g}) / \widetilde{I})_{\bar{f}}$ by the exactness of localisation, it follows from [3, (6.3)] and (i) that $\operatorname{Dim}(U(\mathfrak{g}) / \widetilde{I})=\operatorname{Dim}(H / I)+\operatorname{dim} \mathcal{O}_{\text {min }}$.

5.6. Given a ring $R$ we let $\operatorname{Prim} R$ denote the primitive spectrum of $R$, the set of all primitive ideals of $R$ taken with the Jacobson topology. Set $X:=\operatorname{Prim} U(\mathfrak{g})$ and denote by $X_{\text {fin }}$ the set of all primitive ideals of finite codimension in $U(\mathfrak{g})$. Using the highest weight theory and $[9,(2.5 .6),(3.2 .3)]$ it is easy to observe that $X_{\text {fin }}$ is a countable dense subset of $X$. The topology of $X$ induces that on the complement $X_{\text {inf }}:=X \backslash X_{\text {fin }}$.

Recall that Prim $H$ is a subset of $\operatorname{Spec} H$. By Theorem 5.2(ii), the map $I \mapsto \widetilde{I}$ given by (5.5.1) sets up a bijection between Spec $H$ and the set of all prime ideals of infinite codimension in $U(\mathfrak{g})$. Identify $Z(\mathfrak{g})$ with $Z(H)$ according to Corollary 5.1.vi).

Theorem 5.3. The following are true:

(1) The map $I \mapsto \widetilde{I}$ takes Prim $H$ onto $X_{\text {inf }}$ and induces a homeomorphism of topological spaces $\operatorname{Prim} H \stackrel{\sim}{\rightarrow} X_{\text {inf. }}$

(2) Let $V$ be a finite-dimensional irreducible $H$-module and $I=\operatorname{Ann}_{H} V$. Then $\widetilde{I}=$ $\operatorname{Ann}_{U(\mathfrak{g})}\left(Q_{\chi} \otimes_{H} V\right)$ and $\operatorname{rk}(U(\mathfrak{g}) / \widetilde{I})=\operatorname{dim} \mathrm{Wh}\left(Q_{\chi} \otimes_{H} V\right)=\operatorname{dim} V$.

(3) Let $\mathcal{I}$ be a primitive ideal of $U(\mathfrak{g})$ with $\mathcal{V A}(\mathcal{I})=\overline{\mathcal{O}}_{\text {min. }}$. Then there is a finite-dimensional irreducible $H$-module $E$ such that $\mathcal{I}=\operatorname{Ann}_{U(\mathfrak{g})}\left(Q_{\chi} \otimes_{H} E\right)$.

(4) Let $V_{1}$ and $V_{2}$ be two finite-dimensional irreducible $H$-modules. Then $V_{1} \cong V_{2}$ as $H$-modules if and only if $\operatorname{Ann}_{U(\mathfrak{g})}\left(Q_{\chi} \otimes_{H} V_{1}\right)=\operatorname{Ann}_{U(\mathfrak{g})}\left(Q_{\chi} \otimes_{H} V_{2}\right)$.

(5) For any algebra homomorphism $\eta: Z(\mathfrak{g}) \rightarrow \mathbb{k}$ there is a bijection between the isoclasses of finite-dimensional irreducible $H$-modules with central character $\eta$ and the primitive ideals $\mathcal{I}$ of $U(\mathfrak{g})$ with $\mathcal{I} \cap Z(\mathfrak{g})=\operatorname{Ker} \eta$ and $\mathcal{V} \mathcal{A}(\mathcal{I})=\overline{\mathcal{O}}_{\text {min }}$.

(6) A prime ideal $I$ of $H$ is primitive if and only if $I \cap Z(H)$ is a maximal ideal of $Z(H)$. 
Proof. (a) Let $\left\{J^{\alpha} \mid \alpha \in A\right\}$ be a set of two-sided ideals of $H$, and $J=\bigcap_{\alpha \in A} J^{\alpha}$. Since any two-sided ideal of $H$ is $\sigma$-stable, we have

$$
J_{ \pm}=\{x \pm \sigma(x) \mid x \in J\}=\bigcap_{\alpha \in A}\left\{x \pm \sigma(x) \mid x \in J^{\alpha}\right\}=\bigcap_{\alpha \in A} J_{ \pm}^{\alpha} .
$$

Using 5 5.5.1 it is now easy to deduce that $\widetilde{J}_{f}=\bigcap_{\alpha \in A} \widetilde{J}_{f}^{\alpha}$ and $\widetilde{J}=\bigcap_{\alpha \in A} \widetilde{J}^{\alpha}$. Arguing similarly and using Theorem 5.2 one also observes that given $I, J \in \mathrm{Spec} H$ one has $I \subsetneq J$ if and only if $\widetilde{I} \subsetneq \widetilde{J}$.

(b) Let $I \in \operatorname{Prim} H$. Then $I=\operatorname{Ann}_{H} M_{0}$ for some irreducible $H$-module $M_{0}$. Let $M=$ $Q \otimes_{H} M_{0}$ and identify $M$ with $\mathbb{k}\left[h, z_{1}, \ldots, z_{s}\right] \otimes M_{0}$; see 5.1 for details. Theorem 5.2 shows that $\operatorname{Ann}_{U(\mathfrak{g})} M=\widetilde{J}$ for some $J \in \operatorname{Spec} H$, while Theorem 5.1 yields $\widetilde{J}_{f}=I_{f}$. Then $I=J$ in view of Theorem 5.2(i), forcing $\widetilde{I}=\operatorname{Ann}_{U(\mathfrak{g})} M$. As a consequence, the map $I \mapsto \widetilde{I}$ takes Prim $H$ into $X_{\text {inf. }}$

(c) Now suppose that $\tilde{I} \in X_{\text {inf }}$ for some two-sided ideal $I$ of $H$. By Theorem 5.2 (ii), $I \in \operatorname{Spec} H$. Let $\mathfrak{I}=\{J \in \operatorname{Spec} H \mid J \supsetneq I\}$, and $J_{0}=\bigcap_{J \in \mathfrak{I}} J$. Our discussion in part (a) in conjunction with Theorem 5.2 (ii) implies that $\widetilde{I}:=\{\widetilde{J} \mid J \in \mathfrak{I}\}$ coincides with the set of all prime ideals of $U(\mathfrak{g})$ containing $\widetilde{I}$ properly, and $\widetilde{J}_{0}=\bigcap_{\mathcal{I} \in \widetilde{I}} \mathcal{I}$. Since $\widetilde{I}$ is a primitive ideal, [9, (8.5.7)] applies, yielding $\widetilde{J}_{0} \supsetneq \widetilde{I}$. But then $J_{0} \supsetneq I$ by our concluding remark in part (a).

(d) We claim that the prime ideal $I$ from part (c) is the intersection of some primitive ideals of $H$. To see this one can mimic the proof of Proposition 3.1.15 in [9] which deals with enveloping algebras but applies to a larger class of filtered rings. For the reader's convenience we include the argument which goes back to Duflo. As in [9] we put $B:=$ $H / I$, let $X$ be a variable, and set $C:=B \otimes \mathbb{k}[X]$. The Kazhdan filtration of $H$ induces a filtration of $B$, which in turn gives rise to a filtration of $C$. Since gr $H$ is finitely generated and commutative, by [31, Theorem 4.6(iii)], so are gr $B$ and $\operatorname{gr} C$. Let $a \in J(B)$ where $J(B)$ is the Jacobson radical of $B$.

Suppose $C(1-a X) \neq C$. By Zorn's lemma, there exists a maximal left ideal of $C$ containing $C(1-a X)$, say $L$. Put $M:=C / L$, a simple $C$-module, and let $m_{0}$ denote the image of $1 \in C$ in $M$. Then $m_{0} \neq 0$ and $(1-a X) m_{0}=0$. Let $x$ and $a_{M}$ denote the images of $X$ and $a$ in End $M$. Since $X \in Z(C)$, we have $x \in \operatorname{End}_{C} M$. Since $\operatorname{gr} C$ is finitely generated and commutative, $x$ is invertible in End $C M$ and algebraic over $\mathbb{k}$, by Quillen's lemma. Put $y:=x^{-1}$. Then $x=p(y)$ for some $p \in \mathbb{k}[X]$, and $a_{M}\left(m_{0}\right)=y\left(m_{0}\right)$. Therefore, $(1-a p(a)) m_{0}=(1-y p(y))\left(m_{0}\right)=0$. On the other hand, $a p(a) \in J(B)$, hence $1-a p(a)$ is invertible in $C$; see $[9,(3.1 .12)]$ for instance. This contradiction shows that $C(1-a X)=C$.

As a consequence, $\left(a_{0}+a_{1} X+\cdots+a_{n} X^{n}\right)(1-a X)=1$ for some $a_{i} \in B$. Easy induction on $i$ gives $a_{i}=a^{i}$ for $0 \leq i \leq n$. Then $a^{n+1}=0$, showing that all elements in $J(B)$ are nilpotent. As (0) is a prime ideal of $B=H / I$, it follows from [9, (3.1.14)] that $\bigcap_{J \in \operatorname{Prim} B} J=J(B)=0$. But then $I$ is the intersection of some primitive ideals of $H$, as claimed. 
If $I \notin \operatorname{Prim} H$, then all primitive ideals of $H$ containing $I$ lie in $\mathfrak{I}$. However, this is impossible as $J_{0} \supsetneq I$; see part (c). Thus $I$ must be a primitive ideal of $H$, showing that the map $I \mapsto \widetilde{I}$ induces a bijection between Prim $H$ and $X_{\text {inf }}$; call it $\varkappa$.

Let $\mathcal{Y}$ be a closed set in Prim $H$. Then there is a two-sided ideal $I \mathcal{Y}$ of $H$ such that $\mathcal{Y}=\{J \in \operatorname{Prim} H \mid J \supseteq I \mathcal{Y}\}$; see [9, (3.2.3)]. Our earlier remarks in the proof show that $\varkappa(\mathcal{Y})=\left\{\mathcal{I} \in X_{\text {inf }} \mid \mathcal{I} \supseteq \widetilde{I} \mathcal{Y}\right\}$. Therefore, $\varkappa$ : Prim $H \rightarrow X_{\text {inf }}$ is a closed map.

Recall that the topology on $X_{\text {inf }}$ is induced by the Jacobson topology on Prim $U(\mathfrak{g})$. From $[9,(3.1 .10)]$ it follows that Prim $U(\mathfrak{g})$ is a Zariski space, that is, any closed set in Prim $U(\mathfrak{g})$ is a finite union of irreducible closed sets. But then $X_{\text {inf }}$ is a Zariski space as

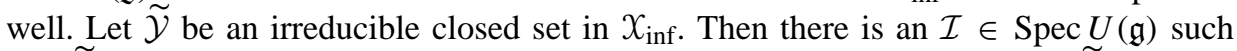
that $\tilde{\mathcal{Y}}=\left\{\mathcal{J} \in X_{\text {inf }} \mid \mathcal{J} \supseteq \mathcal{I}\right\}$; see [9, (3.2.5)]. By Theorem 5.2 (ii), $\mathcal{I}=\widetilde{I}$ for some $I \in$ Spec $H$. Furthermore, $\varkappa^{-1}(\tilde{\mathcal{Y}})=\{J \in$ Prim $H \mid J \supseteq I\}$ by our remarks earlier in the proof. From this it is immediate that $\varkappa^{-1}: X_{\text {inf }} \rightarrow$ Prim $H$ is a closed map too, proving (1).

(e) Let $V$ be a finite-dimensional irreducible $H$-module and $I=\operatorname{Ann}_{H} V$, a primitive ideal of finite codimension in $H$. By part (b) of this proof, $\widetilde{I}=\operatorname{Ann}_{U(\mathfrak{g})} \widetilde{V}$ where $\widetilde{V}=$ $Q_{\chi} \otimes_{H} V$. As before, we identify $\widetilde{V}$ with $\mathbb{k}\left[h, z_{1}, \ldots, z_{s}\right] \otimes V$; see 5.1 Then $\widetilde{\mathcal{B}}_{0}:=$ $U(\mathfrak{g})_{f} / \widetilde{I}_{f}$ identifies with a subalgebra of $\widetilde{\mathcal{B}}:=\mathcal{A}_{e} \otimes(H / I)$. More precisely, from part (b) of the proof of Theorem 5.2 we know that $\tau \otimes \sigma$ acts on $\widetilde{\mathcal{B}}$, and $\widetilde{\mathcal{B}}_{0}=\widetilde{\mathcal{B}}^{\tau \otimes \sigma}$.

Since $U(\mathfrak{g})$ is a completely reducible ad $\mathfrak{g}$-module, $(U(\mathfrak{g}) / \widetilde{I})^{\mathfrak{n}_{\chi}} \cong U(\mathfrak{g})^{\mathfrak{n}_{\chi}} / \widetilde{I}^{\mathfrak{n}_{x}}$; see $[13,(3.2)]$ for example. Since $f$ is central in $U(\mathfrak{g})^{\mathfrak{n}_{\chi}}$, we then have

$$
\mathcal{B}_{0}:=\widetilde{\mathcal{B}}_{0}^{\mathfrak{n}_{x}} \cong\left((U(\mathfrak{g}) / \widetilde{I})_{\bar{f}}\right)^{\mathfrak{n}_{x}} \cong\left(U(\mathfrak{g})^{\mathfrak{n}_{x}}\right)_{f} /\left(U(\mathfrak{g})^{\mathfrak{n}_{\chi}}\right)_{f} \cap \widetilde{I}_{f}
$$

where $\bar{f}$ is the image of $f$ in $U(\mathfrak{g}) / \widetilde{I}$. Put $\bar{H}=H / I$ and $\mathcal{B}=\widetilde{\mathcal{B}}^{\mathfrak{n}_{\chi}}$. Corollary 5.1.iii) implies that $\widetilde{\mathcal{B}}=\widetilde{\mathcal{B}}_{0} \oplus \widetilde{\mathcal{B}}_{0} t$ where $t$ stands for the image of $\widehat{\Delta}$ in $\widetilde{\mathcal{B}}$. Since $\widehat{\Delta}$ commutes with $\mathfrak{n}_{\chi}$, it must be that $\mathcal{B}=\mathcal{B}_{0} \oplus \mathcal{B}_{0} t$. In conjunction with Corollary 5.1 (v) this shows that the natural map $\left(\mathcal{A}_{e} \otimes H\right)^{\mathfrak{n}_{\chi}} \rightarrow \mathcal{B}$ is surjective and $\mathcal{B}=\mathbb{k}\left[t, t^{-1}\right] \otimes H$ as algebras, where $\mathbb{k}\left[t, t^{-1}\right]$ is the Laurent polynomial ring in $t$ over $\mathbb{k}$. As $\bar{H} \cong \operatorname{End}_{\mathbb{k}} V$ is a prime ring, so too is $\mathcal{B}=\bar{H}\left[t, t^{-1}\right]$.

Since $\widetilde{I}$ is a prime ideal of $U(\mathfrak{g})$, the $\operatorname{ring}(U(\mathfrak{g}) / \widetilde{I})^{\mathfrak{n}_{\chi}}$ is prime with $\operatorname{rk}(U(\mathfrak{g}) / I)^{\mathfrak{n}_{\chi}}=$ $\operatorname{rk}(U(\mathfrak{g}) / \widetilde{I})$; see $[13,(13.10)]$. Applying [9, (3.6.15)] we derive that the ring $\mathcal{B}_{0}=$ $\left((U(\mathfrak{g}) / I)^{\mathfrak{n}_{x}}\right)_{\bar{f}}$ is prime with $\operatorname{rk}\left(\mathcal{B}_{0}\right)=\operatorname{rk}(U(\mathfrak{g}) / \widetilde{I})$. On the other hand, it follows from [34] that $\operatorname{rk}(\mathbb{k}[t] \otimes \bar{H})=\operatorname{rk}(\bar{H})$. As $\mathbb{k}\left[t, t^{-1}\right] \otimes \bar{H}$ is a localisation of $\mathbb{k}[t] \otimes \bar{H}$ with respect to the Ore set $\left\{t^{i} \mid i \in \mathbb{Z}_{+}\right\}$, we have

$$
\operatorname{dim} V=\operatorname{rk}(\bar{H})=\operatorname{rk}\left(\mathbb{k}\left[t, t^{-1}\right] \otimes \bar{H}\right)=\operatorname{rk}(\mathcal{B}) ;
$$

see [25, Lemma 2.2.12] for example. We thus need to show that $\operatorname{rk}(\mathcal{B})=\operatorname{rk}\left(\mathcal{B}_{0}\right)$.

It should be mentioned that our present setting (involving a quadratic extension of rings) resembles that of $[16,(6.5)]$ where the above equality has been claimed in a more general situation. However, the proof of Lemma 6.5 in [16] is based on a faulty argument. Moreover, an example involving the first Weyl field shows that under the assumptions of $[16,(6.5)]$ the Goldie ranks of $\mathcal{B}$ and $\mathcal{B}_{0}$ can be different (I am thankful to Anthony 
Joseph for this clarification). We are lucky here because in the present case one can use another argument to prove the required equality of Goldie ranks.

Put $K:=\mathbb{k}(t), K_{0}:=\mathbb{k}\left(t^{2}\right)$, and $S:=\mathbb{k}\left[t^{2}\right] \backslash\{0\}$, a central Ore set in $\mathcal{B}_{0}$ and $\mathcal{B}$. Put $B:=S^{-1} \mathcal{B}$ and $B_{0}:=S^{-1} \mathcal{B}_{0}$. Since $\mathcal{B}=\bar{H}\left[t, t^{-1}\right]$ and $S^{-1} \mathbb{k}\left[t, t^{-1}\right]=K$, we have $B \cong K \otimes_{\mathbb{k}} \bar{H} \cong \operatorname{Mat}_{n}(K)$ where $n=\operatorname{dim} V$. Since $B$ is a simple Artinian ring, all regular elements of $B$ are invertible. Since $S$ consists of regular elements of $\mathcal{B}$, the universality property of quotient rings yields $B \cong \mathcal{Q}(\mathcal{B})$. In particular, $K$ is the Goldie field of $\mathcal{B}$ (this argument provides another proof for the equality $\operatorname{rk}(\mathcal{B})=\operatorname{dim} V)$. As in $[16,(6.5)]$, we regard $B$ as a Galois extension of $B_{0}$. The involution $\tau \otimes \sigma$ induces a $K_{0}$-automorphism of $B$, call it $\iota$. It is easy to see that $\iota(t)=-t, B=B_{0} \oplus B_{0} t$, and $B_{0}=B^{\iota}$. Thus $\iota$ can be viewed as the generator of the Galois group $\operatorname{Gal}\left(K / K_{0}\right)$.

Note that $Z\left(B_{0}\right)=Z(B)^{\iota}=K^{\iota}=K_{0}$ and $B \cong B_{0} \otimes_{K_{0}} K$ as $K$-algebras. Since $\mathcal{B}_{0}$ is a prime ring, so too is $B_{0}$; see [9, (3.6.15)]. The preceding remark then shows that $B_{0}$ is a simple algebra finite-dimensional over its centre $K_{0}$. Since $\mathbb{k}$ is algebraically closed, $K_{0} \cong \mathbb{k}(t)$ is a $C_{1}$-field, by Tsen's theorem. Therefore, $B_{0} \cong \operatorname{Mat}_{m}\left(K_{0}\right)$ as $K_{0}$-algebras. As in the previous paragraph one observes that $B_{0} \cong \mathcal{Q}\left(\mathcal{B}_{0}\right)$ and $K_{0}$ is the Goldie field of $\mathcal{B}_{0}$. Since $B \cong B_{0} \otimes_{K_{0}} K$, one has $m=n$, proving (2).

(f) Let $\mathcal{I}$ be a primitive ideal of $U(\mathfrak{g})$ with $\mathcal{V A}(\mathcal{I})=\overline{\mathcal{O}}_{\text {min }}$. Then $\mathcal{I}=\widetilde{I}$ for some $I \in \operatorname{Prim} H$, by part (1) of this theorem. Thanks to Theorem 5.2 (ii), $\operatorname{Dim}(H / I)=0$. Hence $H / I$ is finite-dimensional over $k$; see $[25,(8.1 .17)]$ for example. Since $H / I$ is a prime ring, it must be that $H / I \cong \operatorname{End}(E)$ for some finite-dimensional $H$-module $E$. As $H / I$ is simple, $I=\operatorname{Ann}_{H} E$. But then $\mathcal{I}=\operatorname{Ann}_{U(\mathfrak{g})}\left(Q_{\chi} \otimes_{H} E\right)$ by part (b) of this proof, as stated in (3).

Now let $V_{1}$ and $V_{2}$ be two finite-dimensional irreducible $H$-modules, and set $I_{i}:=$ $\operatorname{Ann}_{H} V_{i}, i=1,2$. If $V_{1} \cong V_{2}$ as $H$-modules then, of course, $I_{1}=I_{2}$. Hence $\widetilde{I}_{1}=\widetilde{\widetilde{I}}_{2}$, yielding $\operatorname{Ann}_{U(\mathfrak{g})}\left(Q_{\chi} \otimes_{H} V_{1}\right)=\operatorname{Ann}_{U(\mathfrak{g})}\left(Q_{\chi} \otimes_{H} V_{2}\right)$, again by part (b). Conversely, if $\operatorname{Ann}_{U(\mathfrak{g})}\left(Q_{\chi} \otimes_{H} V_{1}\right)=\operatorname{Ann}_{U(\mathfrak{g})}\left(Q_{\chi} \otimes_{H} V_{2}\right)$, then $I_{1}=I_{2}$ in view of Theorem 5.2 (ii) and part (b). So $H / I_{1}=H / I_{2} \cong \mathrm{Mat}_{m}(\mathbb{k})$ for some $m$. It is now straightforward to see that $V_{1} \cong V_{2}$ as $H$-modules, giving (4).

Fix an algebra homomorphism $\eta: Z(\mathfrak{g}) \rightarrow \mathbb{k}$ and identify $Z(\mathfrak{g})$ with $Z(H)$; see Corollary 5.1.(vi). If $V$ is a finite-dimensional $H$-module with central character $\eta$ and $I=\operatorname{Ann}_{H} V$, then $I \cap Z(H)=\operatorname{Ker} \eta$ is a maximal ideal of $Z(H)$, by Schur's lemma. Hence Ker $\eta=\operatorname{Ann}_{U(\mathfrak{g})}\left(Q_{\chi} \otimes_{H} V\right) \cap Z(\mathfrak{g})=\widetilde{I} \cap Z(\mathfrak{g})$. Thanks to Theorem 5.2(ii) we also have $\mathcal{V A}(\widetilde{I})=\overline{\mathcal{O}}_{\text {min }}$. Now let $\mathcal{I} \in X$ be such that $Z(\mathfrak{g}) \cap \mathcal{I}=\operatorname{Ker} \eta$ and $\mathcal{V A}(\mathcal{I})=\overline{\mathcal{O}}_{\text {min }}$. By parts (3) and (4) of this theorem, $\mathcal{I}=\operatorname{Ann}_{U(\mathfrak{g})}\left(Q_{\chi} \otimes_{H} E\right)$ for some finite-dimensional irreducible $H$-module $E$, which is uniquely determined up to isomorphism. Since Ker $\eta \subset \mathcal{I}$ and $Z(\mathfrak{g})=Z(H)$, the $H$-module $E$ has central character $\eta$. We obtain (5).

(g) Let $I \in \underset{\sim}{\operatorname{Spec} H}$ and suppose that $I \cap Z(H)$ is a maximal ideal of $Z(H)$. By Theo$\operatorname{rem} 5.2$ (ii), $\widetilde{I} \in \operatorname{Spec} U(\mathfrak{g})$. As explained in the proof of Corollary 5.1.vi),

$$
Z(H)=Z\left(\mathcal{A}_{e} \otimes H\right)=\mathbb{k} \otimes\left(H^{\sigma} \cap Z(H)\right)=\left(Z\left(\mathcal{A}_{e} \otimes H\right)\right)^{\tau \otimes \sigma} \subseteq Z\left(U(\mathfrak{g})_{f}\right)=Z(\mathfrak{g}) .
$$


It follows that $I \cap Z(H) \subseteq\left(\mathbb{k} \otimes I_{+}\right) \cap Z(\mathfrak{g}) \subseteq \widetilde{I}_{f} \cap Z(\mathfrak{g})=\widetilde{I} \cap Z(\mathfrak{g})$. As $Z(\mathfrak{g})=Z(H)$, we deduce that $\widetilde{I} \cap Z(\mathfrak{g})$ is a maximal ideal of $Z(\mathfrak{g})$. But then $\widetilde{I} \in \mathcal{X}$; see $[9,(8.5 .7)]$. Since $\widetilde{I}$ has infinite codimension in $U(\mathfrak{g})$, part (1) of this theorem implies that $I=\varkappa^{-1}(\widetilde{I}) \in$ Prim $H$. Finally, suppose $I \in \operatorname{Prim} H$ and let $M$ be an irreducible $H$-module such that $I=\operatorname{Ann}_{H} M$. Since gr $H$ is finitely generated and commutative, Quillen's lemma shows that $Z(H)$ acts on $M$ as scalar operators. Consequently, $I \cap Z(H)$ is a maximal ideal of $Z(H)$. The proof of the theorem is now complete.

5.7. Before finishing this section off we wish to discuss a possible extension of the above results to the case of a general algebra $H_{\chi}$. Let $e$ be any nilpotent element in $\mathfrak{g}$ and $\chi=\chi_{e} \in \mathfrak{g}^{*}$. Put $\mathcal{O}=\mathcal{O}_{\chi}$ and denote by $\chi_{\mathcal{O}}$ the set of all primitive ideals $\mathcal{I}$ of $U(\mathfrak{g})$ with $\mathcal{V} \mathcal{A}(\mathcal{I}) \supset \mathcal{O}$. Take Prim $H_{\chi}$ with the Jacobson topology and $x_{\mathcal{O}}$ with the topology induced by that of $\mathcal{X}$.

Question 5.1. Are the following true?

1. The centre of $H_{\chi}$ coincides with the image of $Z(\mathfrak{g})$ in $H_{\chi} 1^{1}$

2. There exists a homeomorphism $\varkappa$ : Prim $H_{\chi} \rightarrow X_{\mathcal{O}}$ such that:

(a) $\operatorname{Dim}(U(\mathfrak{g}) / \varkappa(I))=\operatorname{Dim}(H / I)+\operatorname{dim} \mathcal{O}$ for all $I \in \operatorname{Prim} H_{\chi}$;

(b) $\operatorname{rk}(U(\mathfrak{g}) / \varkappa(I))=\sqrt{\operatorname{dim}_{\mathbb{k}}(H / I)}$ for all $I \in \operatorname{Prim} H_{\chi}$ with $\operatorname{codim}_{H_{\chi}} I<\infty$.

3. For every character $\eta$ of $Z\left(H_{\chi}\right)=Z(\mathfrak{g})$ the map $\varkappa$ induces a bijection between the isoclasses of finite-dimensional $H_{\chi}$-modules with central character $\eta$ and the primitive ideals $\mathcal{I}$ of $U(\mathfrak{g})$ with $\mathcal{I} \cap Z(\mathfrak{g})=\operatorname{Ker} \eta$ and $\mathcal{V} \mathcal{A}(\mathcal{I})=\overline{\mathcal{O}}$.

\section{The Joseph ideal and a presentation of $H$}

6.1. In his seminal work [14] Joseph has discovered that outside type A the enveloping algebra $U(\mathfrak{g})$ has a unique completely prime primitive ideal whose associated variety is $\overline{\mathcal{O}}_{\text {min }}$. This ideal is denoted $\mathcal{J}_{0}$ and referred to as the Joseph ideal of $U(\mathfrak{g})$. For $\mathfrak{g}$ of type A the completely prime primitive ideals of $U(\mathfrak{g})$ with $\mathcal{V A}(\mathcal{I})=\overline{\mathcal{O}}_{\text {min }}$ form a single family parametrised by the elements of $\mathbb{k}$ (this will be explained in more detail in the course of proving Theorem 6.1.

1 At the Oberwolfach meeting on enveloping algebras in March 2005 Victor Ginzburg has explained to me that this is a consequence of the finiteness of the number of symplectic leaves of $\mathcal{S}_{e}$ contained in the fibres of the morphism $f: \mathcal{S}_{e} \rightarrow \mathfrak{g} / / G$ iduced by the adjoint quotient map of $\mathfrak{g}$. Each homogeneous element $z \in \operatorname{gr} Z\left(H_{\chi}\right)$ lies in the Poisson centre of gr $H_{\chi}=\mathbb{k}\left[\mathcal{S}_{e}\right]$, hence reduces to scalars on all symplectic leaves of $\mathcal{S}_{e}$. The Poisson structure on $\mathcal{S}_{e}$ induced by multiplication in $H_{\chi}$ is determined in [10, (3.2), (5.5)]. By [31, (5.4), (6.3)], all scheme-theoretic fibres of $f$ are reduced and irreducible, and $\operatorname{gr} H_{\chi}$ is a flat module over $\operatorname{gr} Z(\mathfrak{g})$. These results are needed to carry out Ginzburg's argument: Since each fibre of $f$ contains a Zariski dense symplectic leaf of $\mathcal{S}_{e}$, the regular function $z$ is constant on each fibre of $f$. The flatness of the $\operatorname{gr} Z(\mathfrak{g})$-module gr $H_{\chi}$ along with the fact that all scheme-theoretic fibres of $f$ are reduced then yields $z \in \operatorname{gr} Z(\mathfrak{g})$, implying $Z\left(H_{\chi}\right)=Z(\mathfrak{g})$. 
The Joseph ideal is prominent in several areas of representation theory, especially in the theory of minimal representations of $p$-adic groups. Different realisations of $\mathcal{J}_{0}$ can be found in the literature for various types of $\mathfrak{g}$ but most of them are ad hoc. This seems almost inevitable as outside type A the orbit $\mathcal{O}_{\text {min }}$ is rigid, that is, forms a single sheet in $\mathfrak{g}^{*}$. Hence $\mathcal{J}_{0}$ cannot be obtained by parabolic induction from a primitive ideal of a proper Levi subalgebra of $\mathfrak{g}$, the only 'regular' way so far to obtain primitive ideals.

It was noticed by Savin (in a letter to Vogan) that Joseph's original proof of the uniqueness of $\mathcal{J}_{0}$ was incomplete. This was recently fixed by W. T. Gan and Savin with the assistance of Wallach; see [11]. The argument in [11] relies on some invariant theory and earlier results of Garfinkle. We shall see in a moment that the existence and uniqueness of $\mathcal{J}_{0}$ follow readily from our results; see also Remark 6.4 .

6.2. Retain the assumptions and conventions of Sections 4 and 5 . Set $\mathbb{k}_{0}:=H / H^{+}$. Since $\mathbb{k}_{0}$ is an irreducible $H$-module, so is the $\mathfrak{g}$-module $Q_{\chi, 0}:=Q_{\chi} \otimes_{H} \mathbb{k}_{0}$. So $J_{0}:=$ $\operatorname{Ann}_{U(\mathfrak{g})} Q_{\chi, 0}$ is a primitive ideal of $U(\mathfrak{g})$.

Proposition 6.1. The ideal $J_{0}$ is completely prime and $\mathcal{V A}\left(J_{0}\right)=\overline{\mathcal{O}}_{\min }$. If $\mathfrak{g}$ is not of type A then $J_{0}$ is the only primitive ideal of $U(\mathfrak{g})$ with these properties, and hence $J_{0}$ is the Joseph ideal in this case.

Proof. Theorem 5.3 (2) shows that $\operatorname{rk}\left(U(\mathfrak{g}) / J_{0}\right)=\operatorname{dim}_{\mathbb{k}} \mathbb{k}_{0}=1$. Hence $\mathcal{Q}\left(U(\mathfrak{g}) / J_{0}\right)$ is a division ring. Then $U(\mathfrak{g}) / J_{0}$ is a domain, that is, $J_{0}$ is completely prime. Theorem 3.1 gives $\mathcal{V} \mathcal{A}\left(J_{0}\right)=\overline{\mathcal{O}}_{\text {min }}$. Now suppose $\mathfrak{g}$ is not of type A. Then Corollary 4.1 implies that $H$ has a unique one-dimensional representation. In view of Theorem 5.3 this means that $U(\mathfrak{g})$ has a unique completely prime primitive ideal whose associated variety is $\overline{\mathcal{O}}_{\text {min }}$. So $J_{0}=\mathcal{J}_{0}$ in this case.

Remark 6.1. The existence part of our proof is hardly shorter than Joseph's original proof in [14] as it relies on the brute force computations of Section 4. However, there is a slightly different proof of the uniqueness of $\mathcal{J}_{0}$ which eludes Section 4 completely. We just sketch the argument leaving the details to the interested reader: If $I$ is an ideal of codimension 1 in $H$ then $[H, H] \subset I$. Since outside type A the Lie algebra $\mathfrak{z}_{\chi}(0)$ is semisimple, we have $\Theta\left(\mathfrak{z}_{\chi}(i)\right) \subset I$ for $i=1$, 2. Also, $C-\mu \in I$ for some $\mu \in \mathbb{k}$. Using Proposition 2.1 it is not hard to observe that $\mu$ is independent of $I$. Therefore, $H$ cannot afford more than one ideal of codimension 1 . The rest of the proof is unchanged.

6.3. In [14], Joseph has also computed the infinitesimal character of $\mathcal{J}_{0}$, that is, the algebra homomorphism $Z(\mathfrak{g}) \rightarrow \mathbb{k}$ through which the centre $Z(\mathfrak{g})$ acts on the primitive quotient $U(\mathfrak{g}) / \mathcal{J}_{0}$. We are going to use his result to determine the elusive constant $c_{0}$ from Proposition 4.1

Theorem 6.1. In the notation of Section 4, the algebra $H$ is generated by the Casimir element $C$ and the subspaces $\Theta\left(\mathfrak{z}_{\chi}(i)\right)$ for $i=0,1$, subject to the following relations:

(i) $\left[\Theta_{x}, \Theta_{y}\right]=\Theta_{[x, y]}$ for all $x, y \in \mathfrak{z}_{\chi}(0)$; 
(ii) $\left[\Theta_{x}, \Theta_{u}\right]=\Theta_{[x, u]}$ for all $x \in \mathfrak{z}_{\chi}(0)$ and $u \in \mathfrak{z}_{\chi}(1)$;

(iii) $C$ is central in $H$;

(iv) $\left[\Theta_{u}, \Theta_{v}\right]=\frac{1}{2}(f,[u, v])\left(C-\Theta_{\text {Cas }}-c_{0}\right)+\frac{1}{2} \sum_{i=1}^{2 s}\left(\Theta_{\left[u, z_{i}\right]^{\sharp}} \Theta_{\left[v, z_{i}^{*}\right]^{\sharp}}+\Theta_{\left[v, z_{i}^{*}\right]^{\sharp}} \Theta_{\left[u, z_{i}\right]^{\sharp}}\right)$ for all $u, v \in \mathfrak{z}_{\chi}(1)$, where $\Theta_{\mathrm{Cas}}=\sum_{i} \Theta_{a_{i}} \Theta_{b_{i}}$ is a Casimir element of the Lie algebra $\Theta\left(\mathfrak{z}_{\chi}(0)\right)$ and the constant $-c_{0}$ is given in the table below:

\begin{tabular}{|c|c|c|c|c|c|c|c|c|c|}
\hline Type & $\mathrm{A}_{n}$ & $\mathrm{~B}_{n}$ & $\mathrm{C}_{n}$ & $\mathrm{D}_{n}$ & $\mathrm{E}_{6}$ & $\mathrm{E}_{7}$ & $\mathrm{E}_{8}$ & $\mathrm{~F}_{4}$ & $\mathrm{G}_{2}$ \\
\hline$-c_{0}$ & $\frac{n(n+1)}{4}$ & $\frac{(2 n+1)(2 n-3)}{4}$ & $\frac{n(2 n+1)}{8}$ & $n(n-2)$ & 36 & 84 & 240 & $\frac{39}{2}$ & $\frac{28}{9}$ \\
\hline
\end{tabular}

If $\mathfrak{g}$ is not of type A then $c_{0}$ is the eigenvalue of $C$ on the primitive quotient $U(\mathfrak{g}) / \mathcal{J}_{0}$. If $\mathfrak{g}$ is of type $\mathrm{A}_{1}$ then $\mathfrak{z}_{\chi}(0)=\mathfrak{z}_{\chi}(1)=0$ and $H=\mathbb{k}[C]$.

Proof. First we determine $c_{0}$. Recall from 4.1 that $(\gamma, \gamma)=2$. Therefore, if $\mathfrak{g}$ is not of type $\mathrm{C}_{n}$ or $\mathrm{G}_{2}$, then the scalar product (., $\left.\cdot\right)$ on the $\mathbb{Q}$-span of $P$ in $\mathfrak{h}^{*}$ coincides with the scalar product $(\cdot \cdot \cdot)$ from Bourbaki's tables in [4]. In the remaining two cases, $(\cdot, \cdot)=$ $\frac{1}{2}(\cdot \cdot \cdot)$ for $\mathfrak{g}$ of type $C_{n}$, and $(\cdot, \cdot)=\frac{1}{3}(\cdot \cdot \cdot)$ for $\mathfrak{g}$ of type $G_{2}$. Recall that for any $\lambda \in \mathfrak{h}^{*}$ the eigenvalue of the Casimir element $C$ on the irreducible highest weight module $L(\lambda)$ equals $(\lambda, \lambda+2 \rho)$.

(a) Suppose $\mathfrak{g}$ is not of type A. In [14, p. 15], Joseph has found an irreducible highest weight module $L\left(\lambda_{0}\right)$ with $\operatorname{Ann}_{U(\mathfrak{g})} L\left(\lambda_{0}\right)=\mathcal{J}_{0}$. It is immediate from the definition of $H^{+}$that $C$ acts on $\mathbb{k}_{0}=H / H^{+}$as scalar $c_{0}$. But then $C_{\mid Q_{0}}=c_{0}$ id. Proposition 6.1 now shows that $C$ acts as $c_{0}$ id on the primitive quotient $U(\mathfrak{g}) / \mathcal{J}_{0}$. In view of our remarks above this yields $c_{0}=\left(\lambda_{0}, \lambda_{0}+2 \rho\right)$.

If $\mathfrak{g}$ is of type $\mathrm{E}$, then $\lambda_{0}=-\varpi_{4}$; see [14]. Using parts (VI) and (VII) of Tables V-VII in [4] one finds that $c_{0}=-240$ for $\mathfrak{g}$ of type $\mathrm{E}_{8}, c_{0}=-84$ for $\mathfrak{g}$ of type $\mathrm{E}_{7}$, and $c_{0}=-36$ for $\mathfrak{g}$ of type $\mathrm{E}_{6}$. If $\mathfrak{g}$ is of type $\mathrm{D}_{n}, n \geq 4$, then $\lambda_{0}=-\varpi_{n-2}$. Parts (VI) and (VII) of Table $\mathrm{V}$ in [4] yield $\left(\varpi_{n-2}, \varpi_{n-2}\right)=n-2$ and $\left(\varpi_{n-2}, 2 \rho\right)=n^{2}-n-2$. Therefore, $c_{0}=-n(n-2)$ in this case.

If $\mathfrak{g}$ is of type $\mathrm{B}_{n}, n \geq 3$, then $\lambda_{0}=-\frac{1}{2}\left(\varpi_{n-2}+\varpi_{n-1}\right)$. Form Table II in [4] we get $\left(\lambda_{0}, \lambda_{0}\right)=(4 n-7) / 4$ and $\left(\lambda_{0}, 2 \rho\right)=-n^{2}+\frac{5}{2}$. Then $c_{0}=-\left(n^{2}-n-\frac{3}{4}\right)=$ $-(2 n+1)(2 n-3) / 4$. If $\mathfrak{g}$ is of type $\mathrm{C}_{n}, n \geq 2$, then $\lambda_{0}=-\frac{1}{2} \varpi_{n}$. Table III in [4] yields $\left(\lambda_{0} \mid \lambda_{0}\right)=n / 4$ and $\left(\lambda_{0} \mid 2 \rho\right)=-n(n+1) / 2$. Consequently, $\left(\lambda_{0} \mid \lambda_{0}+2 \rho\right)=$ $-n(2 n+1) / 4$ and $c_{0}=\frac{1}{2}\left(\lambda_{0} \mid \lambda_{0}+2 \rho\right)=-n(2 n+1) / 8$. If $\mathfrak{g}$ is of type $\mathrm{G}_{2}$, then $\lambda_{0}=-\frac{2}{3} \varpi_{2}=-\frac{2}{3} \widetilde{\alpha}$. Hence $\left(\lambda_{0} \mid \lambda_{0}\right)=\frac{8}{3}$ and

$$
\left(\lambda_{0} \mid 2 \rho\right)=\left(-\frac{2}{3} \varpi_{2} \mid 10 \alpha_{1}+6 \alpha_{2}\right)=-4\left(\varpi_{2} \mid \alpha_{2}\right)=-2\left(\alpha_{2} \mid \alpha_{2}\right)\left\langle\varpi_{2}, \alpha_{2}\right\rangle=-12 ;
$$

see [4, Table IX]. Therefore, $\left(\lambda_{0} \mid \mu_{0}+2 \rho\right)=-\frac{28}{3}$ and $c_{0}=\frac{1}{3}\left(\lambda_{0} \mid \lambda_{0}+2 \rho\right)=-\frac{28}{9}$. If $\mathfrak{g}$ is of type $\mathrm{F}_{4}$, then $\lambda_{0}=-\frac{1}{2}\left(\varpi_{1}+\varpi_{2}\right)$. Using Table VIII in [4] we get $\left(\lambda_{0} \mid \lambda_{0}\right)=\frac{7}{2}$ and $\left(\lambda_{0} \mid 2 \rho\right)=-23$. Hence $c_{0}=\left(\lambda_{0}, \lambda_{0}+2 \rho\right)=\left(\lambda_{0} \mid \lambda_{0}+2 \rho\right)=-\frac{39}{2}$.

(b) Now suppose $\mathfrak{g}$ is of type $\mathrm{A}_{n}, n \geq 2$. This case is more subtle because here we have an infinite family of completely prime ideals in $X$ sharing the same associated variety $\overline{\mathcal{O}}_{\text {min }}$. 
In order to determine $c_{0}$ in the present case we shall have to locate a special member of this family. Theorem 5.3 will play a crucial rôle here.

Let $\mathfrak{p}_{1}$ be the standard parabolic subalgebra of $\mathfrak{g}$ whose Levi subalgebra is generated by $\mathfrak{h}$ and all $e_{ \pm \alpha_{i}}$ with $2 \leq i \leq n$. For any $t \in \mathbb{k}$ the linear function $t \varpi_{1}$ vanishes on all $h_{\alpha_{i}}$ with $i \geq 2$, hence extends uniquely to a one-dimensional representation of $\mathfrak{p}_{1}$. Let $\mathbb{k}_{t}$ denote the corresponding one-dimensional $\mathfrak{p}_{1}$-module, and put $I\left(\mathfrak{p}_{1}, t\right):=$ $\operatorname{Ann}_{U(\mathfrak{g})}\left(U(\mathfrak{g}) \otimes_{U\left(\mathfrak{p}_{1}\right)} \mathbb{k}_{t}\right)$. Although some of the induced $\mathfrak{g}$-modules $U(\mathfrak{g}) \otimes_{U\left(\mathfrak{p}_{1}\right)} \mathbb{k}_{t}$ are reducible, it follows from [9, (8.5.7)] and Conze's theorem [7] that all two-sided ideals $I\left(\mathfrak{p}_{1}, t\right)$ are primitive and completely prime. It is not hard to check that $t_{1} \varpi_{1}$ and $t_{2} \varpi_{1}$ are conjugate under the dot action of the Weyl group $W \cong \mathfrak{S}_{n+1}$ if and only if $t_{1}=t_{2}$. Consequently, all members of the family $\mathfrak{I}_{\mathbb{k}}:=\left\{I\left(\mathfrak{p}_{1}, t\right) \mid t \in \mathbb{k}\right\}$ have pairwise distinct infinitesimal characters. In view of [13, (17.17)] they share the same associated variety $\overline{\mathcal{O}}_{\min }$.

Let $\mathcal{I} \in X$ be a completely prime ideal with $\mathcal{V A}(\mathcal{I})=\overline{\mathcal{O}}_{\text {min }}$. It follows from the main result of Mœglin in [30] that there exist a standard parabolic subalgebra $\mathfrak{p}$ of $\mathfrak{g}$ and a one-dimensional representation $f: \mathfrak{p} \rightarrow \mathbb{k}$ such that $\mathcal{I}=\operatorname{Ann}_{U(\mathfrak{g})}\left(U(\mathfrak{g}) \otimes_{U(\mathfrak{p})} \mathbb{k}_{f}\right)$. In conjunction with [13, (17.17), (15.27)] this yields $\mathcal{I} \in \mathfrak{I}_{\mathbb{k}}$.

Let $I_{0}$ be the two-sided ideal of $H$ generated by $[H, H]$. In order to describe the one-dimensional representations of $H$ we have to take a close look at the commutative $\mathbb{k}$-algebra $H^{\mathrm{ab}}:=H / I_{0}$. Given $x \in H$ we denote by $\bar{x}$ the image of $x$ in $H^{\mathrm{ab}}$. We may assume that $\mathfrak{g}=\mathfrak{s l}_{n+1}(\mathbb{k})$ and $\mathfrak{h}$ is the subalgebra of all diagonal matrices in $\mathfrak{g}$. Let $\left\{e_{i j} \mid 1 \leq i, j \leq n+1\right\}$ be the matrix units in $\mathfrak{g l}_{n+1}(\mathbb{k})$. We may also assume that $\alpha_{i}=\varepsilon_{i}-\varepsilon_{i+1}$ and $e_{\alpha_{i}}=e_{i, i+1}$ for $1 \leq i \leq n$; see Table I in [4]. Then $e=e_{n, n+1}$, $h=e_{n n}-e_{n+1, n+1}$, and $f=e_{n+1, n}$ by our conventions in 4.1 No generality will be lost by assuming that $z_{i}=e_{n+1, i}$ and $z_{i}^{*}=-e_{i n}$ for $1 \leq i \leq n-1$ (notice that $s=n-1$ in the present case). It is straightforward to see that the centre of the subalgebra $\mathfrak{g}(0)^{\sharp}=\mathfrak{z} \chi(0)$ is spanned by the element $z:=e_{n n}+e_{n+1, n+1}-\frac{2}{n+1} I_{n+1}$. Therefore, $\mathfrak{z} \chi(0)=\mathbb{k} z \mathfrak{z}_{\chi}(0)^{\prime}$ where $\mathfrak{z}_{\chi}(0)^{\prime}=\left[\mathfrak{z}_{\chi}(0), \mathfrak{z}_{\chi}(0)\right]$. Since $\mathfrak{z}_{\chi}(1)$ has no zero weight relative to $\mathfrak{h}_{e}=\mathfrak{h} \cap \mathfrak{z}_{\chi}(0)$, this implies that the $\mathbb{k}$-algebra $H^{\mathrm{ab}}$ is generated by $\bar{C}$ and $\bar{\Theta}_{z}$.

Set $u=e_{1, n+1}$ and $v=-e_{n, 1}$. We have $\left[u, z_{i}\right]^{\sharp} \in\left[\mathfrak{z}_{\chi}(0), \mathfrak{z}_{\chi}(0)\right]$ for $2 \leq i \leq s$ and $\left[u, z_{i}^{*}\right]=0$ for $1 \leq i \leq s$. Also, $\left[u, z_{1}\right]^{\sharp}=\left(e_{11}-e_{n+1, n+1}\right)-\frac{1}{2} h=e_{11}-\frac{1}{2}\left(e_{n n}+\right.$ $\left.e_{n+1, n+1}\right)$. Likewise, $\left[v, z_{1}^{*}\right]^{\sharp}=\left(e_{n n}-e_{11}\right)-\frac{1}{2} h=-e_{11}+\frac{1}{2}\left(e_{n n}+e_{n+1, n+1}\right)$. Since $(z, z)=2-\frac{8}{n+1}+\frac{4}{n+1}=\frac{2(n-1)}{n+1}$, we can take $a_{1}=z, b_{1}=\frac{n+1}{2(n-1)} z$, and $a_{i}, b_{i} \in \mathfrak{z} \chi(0)^{\prime}$ for $i>1$. Next observe that $\left(z, e_{11}-\frac{1}{2}\left(e_{n n}+e_{n+1, n+1}\right)\right)=-1$. As $z \perp \mathfrak{z}_{\chi}(0)^{\prime}$, it follows that $e_{11}-\frac{1}{2}\left(e_{n n}+e_{n+1, n+1}\right)$ is congruent to $-\frac{1}{(z, z)} z$ modulo $\mathfrak{z}_{\chi}(0)^{\prime}$. As $(f,[u, v])=1$, Proposition 4.1 now yields

$$
\frac{1}{2}\left(\bar{C}-c_{0}-\frac{n+1}{2(n-1)} \bar{\Theta}_{z}^{2}\right)+\frac{1}{2} \cdot(-2) \cdot \frac{(n+1)^{2}}{4(n-1)^{2}} \bar{\Theta}_{z}^{2}=0
$$

As a consequence, the following relation holds in $H^{\text {ab }}$ :

$$
\bar{\Theta}_{z}^{2}=\frac{(n-1)^{2}}{n(n+1)}\left(\bar{C}-c_{0}\right) .
$$


Since $n \geq 2$, this shows that $H^{\mathrm{ab}}$ is a homomorphic image of the polynomial algebra $\mathbb{k}[X]$. On the other hand, Theorem 5.3 in conjunction with our earlier remarks entails that the one-dimensional representations of $H^{\mathrm{ab}}$ are in 1-1 correspondence with the elements in $\mathfrak{I}_{\mathbb{k}}$. But then the maximal spectrum $\operatorname{Max}\left(H^{\mathrm{ab}}\right)$ of $H^{\mathrm{ab}}$ is infinite, forcing $H^{\mathrm{ab}} \cong \mathbb{k}[X, Y] /\left(X^{2}-\frac{(n-1)^{2}}{n(n+1)} Y\right)$ (under the algebra map $\left.X \mapsto \bar{\Theta}_{z}, Y \mapsto \bar{C}-c_{0}\right)$.

For $c \in \mathbb{k}$ we let $\operatorname{Max}_{c}\left(H^{\mathrm{ab}}\right)$ (resp. $\left.\mathfrak{I}_{\mathbb{k}, c}\right)$ denote the set of all $I$ in $\operatorname{Max}\left(H^{\mathrm{ab}}\right)$ (resp. $I\left(\mathfrak{p}_{1}, t\right)$ in $\left.\mathfrak{I}_{\mathbb{K}}\right)$ containing $\bar{C}-c$ (resp. $\left.C-c\right)$. It is immediate from 6.3.1) that

$$
\left|\operatorname{Max}_{c}\left(H^{\mathrm{ab}}\right)\right|= \begin{cases}2 & \text { when } c \neq c_{0}, \\ 1 & \text { when } c=c_{0} .\end{cases}
$$

Theorem 5.3 implies that for any $c \in \mathbb{k}$ the map $\varkappa$ : Prim $H \rightarrow X_{\text {inf }}, I \mapsto \widetilde{I}$, induces a bijection between $\operatorname{Max}_{c}\left(H^{\mathrm{ab}}\right)$ and $\mathfrak{I}_{\mathbb{k}, c}$. It is well-known that $C$ acts on the induced module $U(\mathfrak{g}) \otimes_{U\left(\mathfrak{p}_{1}\right)} \mathbb{k}_{t}$ as $\left(t \varpi_{1}, t \varpi_{1}+2 \rho\right)$ id. So $I\left(\mathfrak{p}_{1}, t\right)$ contains $C-\left(t \varpi_{1}, t \varpi_{1}+2 \rho\right)$. It is immediate from [4, Table I] that $\left(t \varpi_{1}, t \varpi_{1}+2 \rho\right)=\frac{n}{n+1} t^{2}+n t$. The equation $\frac{n}{n+1} t^{2}+n t-c=0$ has two distinct roots if and only if $n^{2}+\frac{4 n c}{n+1} \neq 0$. Therefore,

$$
\left|\mathfrak{I}_{\mathbb{k}, c}\right|= \begin{cases}2 & \text { when } c \neq-n(n+1) / 4, \\ 1 & \text { when } c=-n(n+1) / 4 .\end{cases}
$$

But then $\operatorname{Max}_{c_{0}}\left(H^{\mathrm{ab}}\right)$ must be mapped onto $\mathfrak{I}_{\mathbb{k},-n(n+1) / 4}$, forcing $c_{0}=-n(n+1) / 4$.

(c) Now let $\widehat{H}$ be the associative $\mathbb{k}$-algebra generated by an element $\widehat{C}$ and isomorphic copies $\widehat{\Theta}\left(\mathfrak{z}_{\chi}(i)\right)$ of the subspaces $\mathfrak{z}_{\chi}(i)$ with $i=1,2$, subject to the relations (i)-(iv) from the formulation of this theorem. Define an increasing filtration in $\widehat{H}$ by giving $\widehat{C}$ filtration degree 4 , by assigning to all nonzero elements of $\widehat{\Theta}\left(\mathfrak{z}_{\chi}(i)\right)$ filtration degree $i+2$, and by extending to $\widehat{H}$ algebraically.

Choose bases $x_{1}, \ldots, x_{q}$ and $y_{1}, \ldots, y_{2 s}$ in $\mathfrak{z} \chi(0)$ and $\mathfrak{z} \chi(1)$, respectively, and set $X_{i}=\widehat{\Theta}_{x_{i}}$ for $1 \leq i \leq q$ and $Y_{i}=\widehat{\Theta}_{y_{i}}$ for $1 \leq i \leq 2 s$. Let $\widehat{H}^{\prime}$ be the $\mathbb{k}$-span of all monomials $m(\mathbf{a}, \mathbf{b}, l):=X_{1}^{a_{1}} \cdots X_{q}^{a_{q}} \cdot Y_{1}^{b_{1}} \cdots Y_{2 s}^{b_{2 s}} \cdot \widehat{C}^{l}$ with $a_{i}, b_{j}, l \in \mathbb{Z}_{+}$. Note that $m(\mathbf{a}, \mathbf{b}, l)$ has filtration degree $2 \sum a_{i}+3 \sum b_{j}+4 l$. Using the relations (i)-(iv) and induction on the filtration degree of $m(\mathbf{a}, \mathbf{b}, l)$ it is straightforward to see that $\widehat{H}^{\prime}$ is a two-sided ideal of $\widehat{H}$. Since $1 \in \widehat{H}^{\prime}$ it must be that $\widehat{H}=\widehat{H}^{\prime}$.

It follows from Proposition 4.1 and Lemmas 2.4 and 2.5 that there is a surjective algebra homomorphism $f: \widehat{H} \rightarrow H$ such that $f(\widehat{C})=C, f\left(X_{i}\right)=\Theta_{x_{i}}$ for $i \leq q$, and $f\left(Y_{i}\right)=\Theta_{y_{i}}$ for $i \leq 2 s$. Since the vectors $f(m(\mathbf{a}, \mathbf{b}, l))$ are linearly independent in $H$, by [31, Theorem 4.6(ii)], the equality $\widehat{H}=\widehat{H}^{\prime}$ shows that $f$ is injective. But then $\widehat{H} \cong H$, and our proof is complete.

Remark 6.2. We have originally computed the infinitesimal character of $J_{0}$ by using a direct approach in the spirit of Section 4; this was done before we established a link between $H$ and $\mathcal{J}_{0}$. Having established that link we discovered that outside type A our result was consistent with [14, p. 15]. In type A we have found two different proofs yielding the same result. This eventually convinced us that the quadratic relation of Theorem 6.1 was 
correct. Our computations are rather lengthy, especially in type C, and will not be presented here.

Remark 6.3. Suppose $\mathfrak{g}$ is of type $\mathrm{C}_{2}$. Then $\mathfrak{z}_{\chi}(0) \cong \mathfrak{s h}_{2}$ and $\mathfrak{z}_{\chi}(1)$ is an irreducible 2-dimensional $\mathfrak{z}_{\chi}(0)$-module. In this case Theorem 6.1 shows that the algebra $H$ is generated by six elements $e, h, f, u, v, c$, subject to the following relations:

1. $(e, h, f)$ is an $\mathfrak{s l}_{2}$-triple relative to the commutator product in $H$;

2. $[e, u]=0=[f, v],[e, v]=u,[f, u]=v,[h, u]=u,[h, v]=-v$;

3. $[u, v]=e f+f e+\frac{1}{2} h^{2}-\frac{1}{2} c-\frac{5}{8}$;

4. $c$ is central in $H$.

For any $t \in \mathbb{k}$ the factor algebra $H_{t}:=H /(c-t)$ is isomorphic to one of the deformed symplectic oscillator algebras $H_{f}$ studied by Khare in [23] (in type $\mathrm{C}_{n}, n \geq 3$, the defining relations of the algebras $H /(C-t)$ differ from those of $\left.H_{f}\right)$. Arguing as in the proof of Theorem 6.4 in [31] one can observe that the image of $Z(\mathfrak{g})$ in $H_{t}$ is isomorphic to a polynomial algebra in one variable. It is likely that the centre of $H_{t}$ is generated by that image. It would be very interesting to describe the Goldie field of $H$ in the present case. In view of Corollary 5.1 (iv) this might help to resolve the Gelfand-Kirillov conjecture for $\mathfrak{g}=\mathfrak{s p}_{4}(\mathbb{k})$.

Remark 6.4 (A. Joseph). Assume $\mathfrak{g}$ is not of type A. The argument below gives a short proof of the uniqueness of $\mathcal{J}_{0}$ relying only on the information available at the time when [14] was written. Let $(e, h, f)$ be an $\mathfrak{s l}_{2}$-triple in $\mathfrak{g}$ with $e$ being a highest root vector. Let $\mathfrak{d}=\operatorname{Ker}(\operatorname{ad} h-\mathrm{id}) \oplus \operatorname{Ker}(\operatorname{ad} h-2 \mathrm{id})$, a Heisenberg Lie subalgebra of $\mathfrak{g}$, and $\mathfrak{r}=\mathbb{k} h \oplus \mathfrak{d}$. Let $J$ be a completely prime primitive ideal of $U(\mathfrak{g})$ such that $\operatorname{Dim}(U(\mathfrak{g}) / J)=\operatorname{dim} \mathcal{O}_{\min }$. Since ad $e$ is nilpotent, $U(\mathfrak{g}) / J$ embeds into its localisation $U$ at $e$, which contains the localisation $A$ of $U(\mathfrak{r})$ at $e$. Let $Z$ denote the centraliser of $A$ in $U$. It follows from [14, Lemma 4.1] that $A$ is a localised Weyl algebra with $\operatorname{Dim}(A)=\operatorname{dim} \mathcal{O}_{\text {min }}$. Clearly, $Z$ inherits a filtration from $U(\mathfrak{g})$ such that $\operatorname{gr} Z$ is commutative. Since $\mathfrak{d}$ is the nilradical of a parabolic subalgebra of $\mathfrak{g}$, Hadziev's theorem shows that the algebra gr $Z$ is finitely generated (one also needs the fact that ad $h$ is semisimple). Since $A$ is central simple, the multiplication map $Z \otimes A \rightarrow U$ is injective. Since both gr $Z$ and gr $A$ are commutative and finitely generated, we have $\operatorname{Dim}(Z \otimes A)=\operatorname{Dim}(Z)+\operatorname{Dim}(A)$. As $\operatorname{Dim}(U)=\operatorname{Dim}(A)$, we get $\operatorname{Dim}(Z)=0$. Hence $Z$ is algebraic over $\mathbb{k}$. Since $U$ is a domain, we now obtain $Z=\mathbb{k}$. Since $\mathfrak{d}$ consists of nilpotent elements of $\mathfrak{g}$, Taylor's lemma proved in [15] implies that for any $h$-weight vector $u \in U$ there is an $h$-weight vector $a \in A$ of the same weight as $u$ such that $u-a$ commutes with the image of $\mathfrak{d}$ in $U$ (a preprint version of [15] was available since 1973 and is quoted in [14]). Taking $u$ to be the image of $f$ in $U$ we get $e(f-a) \in Z=\mathbb{k}$. But then $f \in A$ and so $U=A$, by the simplicity of $\mathfrak{g}$. Now apply $\left[14\right.$, Theorem 4.3] to deduce the equality $J=\mathcal{J}_{0}$.

Lemma 6.1. If $\mathfrak{g}$ is of type $\mathrm{G}_{2}$ then the algebra $H$ admits a 2-dimensional irreducible representation $\rho$ such that $\rho(C)=-\frac{16}{9}$ id and $\rho\left(\Theta_{u}\right)=0$ for all $u \in \mathfrak{z}_{\chi}(1)$.

Proof. For any $\alpha=m \alpha_{1}+n \alpha_{2} \in \Phi^{+}$we set $e_{m, n}=e_{\alpha}, h_{m, n}=h_{\alpha}$, and $f_{m, n}=e_{-\alpha}$. Recall from (4.1) that $\beta=\alpha_{2}$. It is easy to see that in the present case $\mathfrak{z}_{\chi}(0) \cong \mathfrak{s l}_{2}$ 
and $\mathfrak{z}_{\chi}(1)$ is an irreducible 4-dimensional $\mathfrak{z}_{\chi}(0)$-module. Furthermore, $\mathfrak{z}_{\chi}(0)=\mathbb{k} e_{2,1} \oplus$ $\mathbb{k} h_{2,1} \oplus \mathbb{k} f_{2,1}$. We can assume, after possibly an admissible sign change, that $z_{1}=f_{3,2}$, $z_{1}^{*}=e_{3,1}, z_{2}=f_{1,1}$, and $z_{2}^{*}=\frac{1}{3} e_{1,0}$. Then $\left[e_{3,1}, f_{3,2}\right]=\frac{1}{3}\left[e_{1,0}, f_{1,1}\right]=f_{0,1}=f$. Put $u_{1}^{*}=e_{3,2}, u_{1}=-f_{3,1}, u_{2}^{*}=e_{1,1}$, and $u_{2}=a f_{1,0}$, where $a \in \mathbb{k}^{\times}$. Clearly, $\mathfrak{z}_{\chi}(1)$ is spanned by the $u_{1}, u_{2}, u_{1}^{*}, u_{2}^{*}$. Since

$$
\begin{aligned}
\left(\left[e_{3,2},-f_{3,1}\right],\left[f_{3,2}, e_{3,1}\right]\right) & =\left(e_{3,2},\left[f_{3,2}, h_{3,1}\right]\right)=-\left(e_{3,2},\left[h_{3,1}, f_{3,2}\right]\right) \\
& =\left(e_{3,2}, f_{3,2}\right)=1,
\end{aligned}
$$

there is $a \in \mathbb{k}^{\times}$such that $\left[u_{i}, u_{j}\right]=\left[u_{i}^{*}, u_{j}^{*}\right]=0$ and $\left[u_{i}^{*}, u_{j}\right]=-\delta_{i j} e$ for $1 \leq i, j \leq 2$.

Let $\{E, H, F\}$ be the standard basis of $\mathfrak{s l}_{2}(\mathbb{k})$ and $\mathcal{E}=\Theta\left(\mathfrak{z}_{\chi}(0)\right) \cup \Theta\left(\mathfrak{z}_{\chi}(1)\right) \cup\{C\}$. Let $\rho: \mathcal{E} \rightarrow \mathrm{Mat}_{2}(\mathbb{k})$ be such that $\rho\left(\Theta_{x e_{2,1}+y h_{2,1}+z f_{2,1}}\right)=x E+y H+z F, \rho\left(\Theta_{u}\right)=0$, and $\rho(C)=-\frac{16}{9} I_{2}$ for all $x, y, z \in \mathbb{k}$ and $u \in \mathfrak{z}_{\chi}(1)$. We claim that the elements from $\rho(\mathcal{E})$ satisfy the relations (i)-(iv) of Theorem 6.1. Since the relations (i)-(iii) are satisfied for obvious reasons, we just need to check the quadratic relation (iv).

Since $2 \alpha_{1}+\alpha_{2}$ is a short root, we have $\left(h_{2,1}, h_{2,1}\right)=6$ and $\left(e_{2,1}, f_{2,1}\right)=3$. Therefore, $C_{0}=\frac{1}{3}\left(e_{2,1} f_{2,1}+f_{2,1} e_{2,1}+\frac{1}{2} h_{2,1}^{2}\right)$. Note that $E F+F E+\frac{1}{2} H^{2}=\frac{3}{2} I_{2}$. Since $c_{0}=-\frac{28}{9}$ in the present case, we have to show that

$$
\sum_{i=1}^{4}\left(\rho\left(\Theta_{\left[u, z_{i}\right]^{\sharp}}\right) \rho\left(\Theta_{\left.\left[v, z_{i}^{*}\right]\right)^{\sharp}}\right)+\rho\left(\Theta_{\left[v, z_{i}^{*}\right]^{\sharp}}\right) \rho\left(\Theta_{\left[u, z_{i}\right]^{\sharp}}\right)\right)=-\frac{5}{6}(f,[u, v]) I_{2}
$$

for all $u, v \in \mathfrak{z}_{\chi}(1)$. When $u$ and $v$ run through the set $\left\{u_{1}, u_{2}, u_{1}^{*}, u_{2}^{*}\right\}$, the LHS of 6.3.2 is always a linear combination of matrices $X Y+Y X$ with $X, Y \in \mathfrak{s h}_{2}(\mathbb{k})$. Since all such matrices are multiples of $I_{2}$, the LHS of 6.3.2 equals $g(u, v) I_{2}$ for some skewsymmetric bilinear form $g$ on $\mathfrak{z} \chi(1)$. Using the relations (i) and (ii) of Theorem 6.1 it is easy to observe that this form is $\mathfrak{z}_{\chi}(0)$-invariant. As $\mathfrak{z}_{\chi}(1)$ is an irreducible $\mathfrak{z} \chi(0)$-module, there is a scalar $c \in \mathbb{k}$ such that $g(u, v)=c(f,[u, v])$ for all $u, v \in \mathfrak{z} \chi(1)$. Thus we need to check that $c=-\frac{5}{6}$.

Note that $\left[u_{1}^{*}, z_{i}^{*}\right]=\left[u_{1}, z_{i}\right]=0$ for $i=1,2$. Also, $\left[u_{1}^{*}, z_{2}\right]^{\#}=\left[u_{1}^{*}, z_{2}\right]$ and $\left[u_{1}, z_{2}^{*}\right]^{\sharp}=\left[u_{1}, z_{2}^{*}\right]$. It follows that for $i=1,2$,

$$
\begin{aligned}
\left(\left[u_{1}^{*}, z_{i}\right],\left[u_{1}, z_{i}^{*}\right]\right) & =\left(u_{1}^{*},\left[u_{1},\left[z_{i}, z_{i}^{*}\right]\right]\right)=-\left(u_{1}^{*},\left[u_{1}, f\right]\right)=-\left(\left[u_{1}^{*}, u_{1}\right], f\right) \\
& =(e, f)=1 .
\end{aligned}
$$

As $\left(u_{1}^{*}, z_{1}\right)=-\left(u_{1}, z_{1}^{*}\right)=1$, we have $\left[u_{1}^{*}, z_{1}\right]^{\sharp}=\left[u_{1}^{*}, z_{1}\right]-\frac{1}{2} h$ and $\left[u_{1}, z_{1}^{*}\right]^{\sharp}=$ $\left[u_{1}, z_{1}^{*}\right]+\frac{1}{2} h$. Consequently,

$$
\begin{aligned}
\left(\left[u_{1}^{*}, z_{1}\right]^{\sharp},\left[u_{1}, z_{1}^{*}\right]^{\sharp}\right) & =\left(\left[u_{1}^{*}, z_{1}\right],\left[u_{1}, z_{1}^{*}\right]\right)+\frac{1}{2}\left(\left[u_{1}^{*}, z_{1}\right], h\right)-\frac{1}{2}\left(h,\left[u_{1}, z_{1}^{*}\right]\right)-\frac{1}{4}(h, h) \\
& =1+\frac{1}{2}\left(u_{1}^{*}, z_{1}\right)-\frac{1}{2}\left(u_{1}, z_{1}^{*}\right)-\frac{1}{2}=2-\frac{1}{2}=\frac{3}{2} .
\end{aligned}
$$

Since $\left[u_{1}^{*}, z_{1}\right]^{\sharp},\left[u_{1}^{*}, z_{2}\right],\left[u_{1}, z_{1}^{*}\right]^{\sharp}$, and $\left[u_{1}, z_{2}^{*}\right]$ are multiples of $h_{2,1}, e_{2,1}, h_{2,1}$, and $f_{2,1}$, respectively, and $\left(e_{2,1}, f_{2,1}\right)=\frac{1}{2}\left(h_{2,1}, h_{2,1}\right)=3$, the preceding remarks show that

$$
\sum_{i=1}^{4}\left(\rho\left(\Theta_{\left[u_{1}^{*}, z_{i}\right]^{\sharp}}\right) \rho\left(\Theta_{\left.\left[u_{1}, z_{i}^{*}\right]\right)^{\sharp}}\right)+\rho\left(\Theta_{\left[u_{1}, z_{i}^{*}\right]^{\sharp}}\right) \rho\left(\Theta_{\left[u_{1}^{*}, z_{i}\right]^{\sharp}}\right)\right)=\frac{1}{3}(E F+F E)+\frac{1}{2} H^{2}=\frac{5}{6} I_{2} .
$$


As $\left(f,\left[u_{1}^{*}, u_{1}\right]\right)=-1$, we deduce from 6.3 .2 that $c=-\frac{5}{6}$, as wanted. As Theorem 6.1 gives a presentation of $H$ by generators and relations, the result follows.

Remark 6.5. It is immediate from the proof of Lemma 6.1 that for $\mathfrak{g}$ of type $\mathrm{G}_{2}$ the following relation holds in $H$ :

$$
\left[\Theta_{u_{1}^{*}}, \Theta_{u_{1}}\right]=-\frac{1}{2} C+\frac{1}{3}\left(\Theta_{e_{2,1}} \Theta_{f_{2,1}}+\Theta_{f_{2,1}} \Theta_{e_{2,1}}+\frac{1}{2} \Theta_{h_{2,1}}^{2}\right)+\frac{1}{6} \Theta_{h_{2,1}}^{2}-\frac{14}{9} .
$$

The expressions for all $\left[\Theta_{u}, \Theta_{v}\right]$ with $u, v \in \mathfrak{z}_{\chi}(1)$ can be derived from 6.3.3) by using the action of ad $\Theta\left(\mathfrak{z}_{\chi}(0)\right)$ on $\Theta\left(\mathfrak{z}_{\chi}(i)\right), i=1,2$. For example, it can be deduced easily that $\left[\Theta_{u_{1}^{*}}, \Theta_{u_{2}^{*}}\right]$ is a nonzero scalar multiple of $\Theta_{e_{2,1}}^{2}$. This implies that the span of all PBW monomials in $C, \Theta_{h_{2,1}}, \Theta_{e_{2,1}}, \Theta_{u_{1}^{*}}, \Theta_{u_{2}^{*}}$ is a subalgebra of $H$. It can be regarded as a Borel subalgebra of $H$.

6.4. As yet another application of Theorems 5.3 and 6.1 we are going to classify all irreducible finite-dimensional representations of $H$ in the case where $\mathfrak{g}$ is of type $\mathrm{C}_{n}$ or $\mathrm{G}_{2}$. Dimension formulae for these representations will be given. We shall rely on Joseph's theory of Goldie-rank polynomials. The reader will notice that our method is quite general and can be applied to any simple Lie algebra $\mathfrak{g}$. However, various problems remain in the general case, especially for Lie algebras of type $E_{7}$ and $E_{8}$. We hope to return to this interesting subject in the future.

Given $v \in \mathfrak{h}^{*}$ we denote by $I(v)$ the annihilator of the irreducible highest weight module $L(v)$ in $U(\mathfrak{g})$. Recall from the proof of Theorem 6.1 that $\lambda_{0}=-\frac{1}{2} \varpi_{n}$ for $\mathfrak{g}$ of type $C_{n}$ and $\lambda_{0}=-\frac{2}{3} \varpi_{2}$ for $\mathfrak{g}$ of type $\mathrm{G}_{2}$. Let $\Phi_{0}=\left\{\alpha \in \Phi \mid\left\langle\lambda_{0}, \alpha\right\rangle \in \mathbb{Z}\right\}$. It is easy to see that $\Phi_{0}$ coincides with the set of all short roots in $\Phi$. In particular, $\Phi_{0}$ is a root system in $\mathfrak{h}^{*}$ but not a closed subsystem of $\Phi$. The set $\Pi_{0}=\left\{\alpha_{1}, \ldots, \alpha_{n-1}, \alpha_{n-1}+\alpha_{n}\right\}$ is the basis of simple roots in $\Phi_{0}$ contained in $\Phi^{+}$. This implies that $\Phi_{0}$ is of type $\mathrm{D}_{n}$ and $\mathrm{A}_{2}$ when $\mathfrak{g}$ is of type $C_{n}$ and $G_{2}$, respectively (our convention here is that $D_{2} \cong A_{1} \times A_{1}$ and $\mathrm{D}_{3} \cong \mathrm{A}_{3}$ ). Note that $\lambda_{0}=-\frac{d-1}{d} \varpi_{n}$ where

$$
d=\frac{\left(\alpha_{n}, \alpha_{n}\right)}{\left(\alpha_{n-1}, \alpha_{n-1}\right)}= \begin{cases}2 & \text { when } \mathfrak{g} \text { is of type } \mathrm{C}_{n} \\ 3 & \text { when } \mathfrak{g} \text { is of type } \mathrm{G}_{2}\end{cases}
$$

It is well-known that the subgroup $W_{0}:=\left\{w \in W \mid w\left(\lambda_{0}\right)-\lambda_{0} \in \mathbb{Z} \Phi\right\}$ of $W$ is generated by the reflections $s_{\alpha}$ with $\alpha \in \Phi_{0}$, hence identifies with the Weyl group of $\Phi_{0}$. We note for further references that

$$
\begin{aligned}
\left\langle\lambda_{0}+\mu+\rho, \alpha_{n-1}+\alpha_{n}\right\rangle & =\left\langle\lambda_{0}+\mu+\rho, \alpha_{n-1}\right\rangle+d\left\langle\lambda_{0}+\mu+\rho, \alpha_{n}\right\rangle \\
& =\left\langle\mu, \alpha_{n-1}\right\rangle+1+d\left(\left\langle\mu, \alpha_{n}\right\rangle-\frac{d-1}{d}+1\right) \\
& =\left\langle\mu, \alpha_{n-1}\right\rangle+d\left\langle\mu, \alpha_{n}\right\rangle+2
\end{aligned}
$$


Theorem 6.2. Let $\Phi_{0}^{+}$denote the set of all short roots in $\Phi^{+}$.

1. If $\mathfrak{g}$ is of type $\mathrm{C}_{n}, n \geq 2$, then to every $\mu \in P^{+}$there corresponds a finite-dimensional simple $H$-module $V_{H}(\mu)$ such that

$$
\operatorname{dim} V_{H}(\mu)=\prod_{\alpha \in \Phi_{0}^{+}} \frac{\left\langle 2 \mu+2 \rho-\varpi_{n}, \alpha\right\rangle}{\left\langle 2 \rho-\varpi_{n}, \alpha\right\rangle} .
$$

Any finite-dimensional simple $H$-module is isomorphic to one of the modules $V_{H}(\mu)$, $\mu \in P^{+}$. The central characters of these modules are pairwise distinct.

2. If $\mathfrak{g}$ is of type $\mathrm{G}_{2}$, then to every $\mu=a \varpi_{1}+b \varpi_{2} \in P^{+}$there correspond two finitedimensional simple $H$-modules $V_{H}^{ \pm}(\mu)$ such that

$$
\begin{aligned}
& \operatorname{dim} V_{H}^{+}(\mu)=\frac{(a+1)(a+3 b+2)(2 a+3 b+3)}{6}, \\
& \operatorname{dim} V_{H}^{-}(\mu)=\frac{(a+1)(a+3 b+3)(2 a+3 b+4)}{6} .
\end{aligned}
$$

Any finite-dimensional simple $H$-module is isomorphic to one of the modules $V_{H}^{ \pm}(\mu)$, $\mu \in P^{+}$. The central characters of these modules are pairwise distinct.

Proof. By Theorem 5.3 the isoclasses of finite-dimensional simple $H$-modules are in 1-1 correspondence with the primitive ideals $\mathcal{I}$ of $U(\mathfrak{g})$ such that $\mathcal{V} \mathcal{A}(\mathcal{I})=\overline{\mathcal{O}}_{\text {min }}$. By Duflo's theorem, $\mathcal{I}=I(\lambda)$ for some $\lambda \in \mathfrak{h}^{*}$. Let $\Phi_{\lambda}=\{\alpha \in \Phi \mid\langle\lambda, \alpha\rangle \in \mathbb{Z}\}$ and let $\Pi_{\lambda}$ be the basis of simple roots of $\Phi_{\lambda}$ contained in $\Phi_{\lambda} \cap \Phi^{+}$. As explained in [19, p. 41], the equality $\mathcal{V} \mathcal{A}(I(\lambda))=\overline{\mathcal{O}}_{\text {min }}$ holds if and only if $\operatorname{dim} \mathcal{O}_{\text {min }}=|\Phi|-\left|\Phi_{\lambda}\right|$ and $\langle\lambda+\rho, \alpha\rangle>0$ for all $\alpha \in \Pi_{\lambda}$ (the argument in [19] relies on the fact that $\mathcal{O}_{\min }$ is not a special orbit in the sense of Lusztig when $\mathfrak{g}$ is not simply laced). Since $\operatorname{dim} \mathcal{O}_{\min }=|\Phi|-\left|\Phi_{0}\right|$, we have $\left|\Phi_{0}\right|=$ $\left|\Phi_{\lambda}\right|$. Now $\Phi_{\lambda}^{\vee}$ is a closed symmetric subsystem of the dual root system $\Phi^{\vee} \subset \mathfrak{h}$. The Borel-de Siebenthal algorithm implies that there is only one such subsystem in $\Phi^{\vee}$ of size $\left|\Phi_{0}\right|$, namely $\Phi_{0}^{\vee}$; see [4, Ch. VI, Sect. 4, Exerc. 4]. This shows $\Phi_{\lambda}=\Phi_{0}$ and $\Pi_{\lambda}=\Pi_{0}$.

Write $\lambda=\lambda_{0}+\sum_{i=1}^{n} l_{i} \varpi_{i}$ with $l_{i} \in \mathbb{k}$. Since $\alpha_{1}, \ldots, \alpha_{n-1} \in \Pi_{0}$, it must be that $l_{i} \in \mathbb{Z}_{+}$for $1 \leq i \leq n-1$, while our earlier remarks show that $\left\langle\lambda+\rho, \alpha_{n-1}+\alpha_{n}\right\rangle=$ $l_{n-1}+d l_{n}+2$ is a positive integer. Hence $l_{n} \in \frac{1}{d} \mathbb{Z}$. Since $\Phi_{\lambda} \neq \Phi$ we have $\lambda \notin P$, giving $l_{n} \notin-\frac{1}{d}+\mathbb{Z}$. For $\mathfrak{g}$ of type $\mathrm{C}_{n}$ this says $l_{n} \in \mathbb{Z}$, while for $\mathfrak{g}$ of type $\mathrm{G}_{2}$ we infer that either $l_{n} \in \mathbb{Z}$ or $l_{n} \in \frac{1}{3}+\mathbb{Z}$.

It is easy to see that $s_{\alpha_{n}}$ permutes the positive short roots in $\Phi$. Therefore, $I(\lambda)=$ $I\left(s_{\alpha_{n}} \cdot \lambda\right)$; see $[13,(5.16)]$. As $\alpha_{n}=-d \varpi_{n-1}+2 \varpi_{n}$ we have

$$
\begin{aligned}
s_{\alpha_{n}} \cdot \lambda & =s_{\alpha_{n}}\left(\sum_{i=1}^{n-1}\left(l_{i}+1\right) \varpi_{i}+\left(l_{n}+\frac{1}{d}\right) \varpi_{n}\right)-\rho=\lambda-\frac{d l_{n}+1}{d} \alpha_{n} \\
& =\lambda_{0}+\sum_{i=1}^{n-2} l_{i} \varpi_{i}+\left(l_{n-1}+d l_{n}+1\right) \varpi_{n-1}+\left(l_{n}-\frac{2}{d}\left(d l_{n}+1\right)\right) \varpi_{n} \\
& =\lambda_{0}+\sum_{i=1}^{n-2} l_{i} \varpi_{i}+\left(l_{n-1}+d l_{n}+1\right) \varpi_{n-1}-\left(l_{n}+\frac{2}{d}\right) \varpi_{n} .
\end{aligned}
$$


Thus replacing $\lambda$ by $s_{\alpha_{n}} \cdot \lambda$ if necessary we may assume further that $l_{n} \geq 0$.

Suppose $l_{n} \in \mathbb{Z}$. Then the above discussion shows that $\lambda-\lambda_{0} \in P^{+}$. It follows from Joseph's theory of Goldie-rank polynomials that

$$
\operatorname{rk}\left(U(\mathfrak{g}) / I\left(\lambda_{0}+\mu\right)\right)=c \prod_{\alpha \in \Phi_{0}^{+}}\left\langle\lambda_{0}+\mu+\rho, \alpha\right\rangle
$$

for all $\mu \in P^{+}$, where $c$ is a constant independent of $\mu$; see [17, p. 303]. Recall that Theorem 5.3 associates to each $\mathcal{I} \in X$ with $\mathcal{V} \mathcal{A}(\mathcal{I})=\overline{\mathcal{O}}_{\text {min }}$ an irreducible finite-dimensional $H$-module (up to isomorphism). Abusing notation we denote this module by $\varkappa^{-1}(\mathcal{I})$. We have already mentioned that $\mathcal{V} \mathcal{A}\left(I\left(\lambda_{0}+\mu\right)\right)=\overline{\mathcal{O}}_{\text {min }}$. Therefore, to each $\mu \in P^{+}$there corresponds an irreducible finite-dimensional $H$-module $V_{H}(\mu):=\varkappa^{-1}\left(I\left(\lambda_{0}+\mu\right)\right)$. By Theorem 5.3 (2),

$$
\operatorname{dim} V_{H}(\mu)=\operatorname{rk}\left(U(\mathfrak{g}) / I\left(\lambda_{0}+\mu\right)\right) \quad\left(\forall \mu \in P^{+}\right) .
$$

Since $I\left(\lambda_{0}\right)$ is the Joseph ideal, Theorem 5.3 together with Proposition 6.1 gives $\operatorname{dim} V_{H}(0)=\operatorname{dim} H / H^{+}=1$. Therefore, $c^{-1}=\prod_{\alpha \in \Phi_{0}^{+}}\left\langle\lambda_{0}+\rho, \alpha\right\rangle$ and

$$
\operatorname{dim} V_{H}(\mu)=\prod_{\alpha \in \Phi_{0}^{+}} \frac{\left\langle\lambda_{0}+\mu+\rho, \alpha\right\rangle}{\left\langle\lambda_{0}+\rho, \alpha\right\rangle}
$$

Since $\lambda_{0}+\rho+P^{+}$is contained in the interior of the dominant Weyl chamber, the modules in the set $\left\{V_{H}(\mu) \mid \mu \in P_{+}\right\}$have pairwise distinct central characters. This settles the case where $\mathfrak{g}$ is of type $\mathrm{C}_{n}$.

Suppose $\mathfrak{g}$ of type $\mathrm{G}_{2}$. For $\mu=a \varpi_{1}+b \varpi_{2} \in P^{+}$we put $V_{H}^{+}(\mu):=V_{H}(\mu)$. Since $\Phi_{0}^{+}=\left\{\alpha_{2}, \alpha_{1}+\alpha_{2}, 2 \alpha_{1}+\alpha_{2}\right\}$ and $\lambda_{0}=-\frac{2}{3} \varpi_{2}$, the dimension formula 6.4.1 reads

$$
\operatorname{dim} V_{H}^{+}(\mu)=\frac{(a+1)(a+3 b+2)(2 a+3 b+3)}{6} .
$$

Now suppose $l_{n} \notin \mathbb{Z}$. Our earlier remarks show that $\mathfrak{g}$ is of type $\mathrm{G}_{2}$ and $l_{n} \in \frac{1}{3}+\mathbb{Z}_{+}$. As a consequence, $\lambda \in \frac{1}{2} \lambda_{0}+P^{+}$. For any $\mu \in P^{+}$we have $\Phi_{\frac{1}{2} \lambda_{0}+\mu}=\Phi_{0}$. As $\frac{1}{2} \lambda_{0}+$ $\mu+\rho$ lies in the interior of the dominant Weyl chamber, the above argument applies, yielding $\mathcal{V} \mathcal{A}\left(I\left(\frac{1}{2} \lambda_{0}+\mu\right)\right)=\overline{\mathcal{O}}_{\text {min }}$. Theorem 5.3 shows that $V_{H}^{-}(\mu):=\varkappa^{-1}\left(I\left(\frac{1}{2} \lambda_{0}+\mu\right)\right)$ is an irreducible finite-dimensional $H$-module with

$$
\operatorname{dim} V_{H}^{-}(\mu)=\operatorname{rk}\left(U(\mathfrak{g}) / I\left(\frac{1}{2} \lambda_{0}+\mu\right)\right) .
$$

In conjunction with the discussion in $[17$, p. 303] this entails that

$$
\operatorname{dim} V_{H}^{-}(\mu)=\operatorname{rk}\left(U(\mathfrak{g}) / I\left(\frac{1}{2} \lambda_{0}+\mu\right)\right)=c^{\prime} \prod_{\alpha \in \Phi_{0}^{+}}\left\langle\frac{1}{2} \lambda_{0}+\mu+\rho, \alpha\right\rangle
$$

for all $\mu \in P^{+}$where $c^{\prime}$ is a constant independent of $\mu$. 
It also follows from Theorem 5.3 that $C$ acts on $V_{H}^{ \pm}(\mu)$ as $f^{ \pm}(\mu)$ id where $f^{+}(\mu)=$ $\left(\lambda_{0}+\mu, \lambda_{0}+\mu+2 \rho\right)$ and $f^{-}(\mu)=\left(\frac{1}{2} \lambda_{0}+\mu, \frac{1}{2} \lambda_{0}+\mu+2 \rho\right)$. We have noted in the proof of Theorem 6.1 that $(\cdot, \cdot)=\frac{1}{3}(\cdot \mid \cdot)$. By $\left[4\right.$, Table IX], $\left(\varpi_{1} \mid \varpi_{1}\right)=2,\left(\varpi_{1} \mid \varpi_{2}\right)=3$, and $\left(\varpi_{2} \mid \varpi_{2}\right)=6$. Using this fact it is straightforward to see that $f^{-}(\mu)=f^{-}\left(a \varpi_{1}+b \varpi_{2}\right)$ is a quadratic polynomial in $a, b$ with all coefficients positive except for the constant term $f^{-}(0)=\left(-\frac{1}{3} \varpi_{2},-\frac{1}{3} \varpi_{2}+2 \rho\right)=-\frac{16}{9}$. Furthermore,

$$
f^{+}\left(a \varpi_{1}+b \varpi_{2}\right)=\frac{2}{3} a^{2}+2 b^{2}+2 a b+2 a+\frac{10}{3} b-\frac{28}{9} .
$$

As a consequence, $f^{+}(\mu)>-\frac{16}{9}$ for all nonzero $\mu \in P^{+}$.

Let $M$ be an $H$-module affording the representation $\rho$ from Lemma 6.1. The above discussion shows that $M \cong V^{ \pm}(v)$ for some $v \in P^{+}$. Since $C$ acts on $M$ as $-\frac{16}{9}$ id, the preceding remark yields $M \cong V_{H}^{-}(0)$. As $\operatorname{dim} M=2$, this allows us to determine the scale factor $c^{\prime}$. In view of 6.4 .2 we then get

$$
\operatorname{dim} V_{H}^{-}(\mu)=2 \prod_{\alpha \in \Phi_{0}^{+}} \frac{\left\langle-\frac{1}{3} \varpi_{2}+\mu+\rho, \alpha\right\rangle}{\left\langle-\frac{1}{3} \varpi_{2}+\rho, \alpha\right\rangle}=\frac{(a+1)(a+3 b+3)(2 a+3 b+4)}{6}
$$

for all $\mu \in P^{+}$. Since $\left(-\frac{2}{3} \varpi_{2}+\rho+P^{+}\right) \cap\left(-\frac{1}{3} \varpi_{2}+\rho+P^{+}\right)=\emptyset$ and the union $\left(-\frac{2}{3} \varpi_{2}+\rho+P^{+}\right) \cup\left(-\frac{1}{3} \varpi_{2}+\rho+P^{+}\right)$is contained in the interior of the dominant Weyl chamber, the modules in the set $\left\{V_{H}^{ \pm}(\mu) \mid \mu \in P^{+}\right\}$have pairwise distinct central characters. This completes the proof.

Remark 6.6. When $\mathfrak{g}$ is of type $\mathrm{C}_{2}$ we have $\Phi_{0}^{+}=\left\{\varepsilon_{1}-\varepsilon_{2}, \varepsilon_{1}+\varepsilon_{2}\right\}, \varpi_{1}=\varepsilon_{1}, \varpi_{2}=$ $\varepsilon_{1}+\varepsilon_{2}$, and $\lambda_{0}=-\frac{1}{2}\left(\varepsilon_{1}+\varepsilon_{2}\right)$; see [4, Table III]. In this case our dimension formula reads

$$
\operatorname{dim} V_{H}(\mu)=\frac{(a+1)(a+2 b+2)}{2}=\frac{(r-s+1)(r+s+2)}{2},
$$

where $\mu=a \varpi_{1}+b \varpi_{2}=(a+b) \varepsilon_{1}+b \varepsilon_{2}=r \varepsilon_{1}+s \varepsilon_{2}$ and $r, s \in \mathbb{Z}_{+}, r \geq s$. The same dimension formula can be found in [23] where it was obtained by a completely different method in the context of deformed symplectic oscillator algebras of rank one; see Remark 6.3.

\section{Highest weight modules for $H$}

7.1. Let $\Phi_{e}$ denote the set of all $\alpha \in \Phi$ with $\alpha(h) \in\{0,1\}$, and put $\Phi_{e}^{ \pm}=\Phi_{e} \cap \Phi^{ \pm}$, $\Phi_{e, i}^{ \pm}=\left\{\alpha \in \Phi_{e}^{ \pm} \mid \alpha(h)=i\right\}$. Recall that $\mathfrak{z}_{x}$ is spanned by $\mathfrak{h}_{e}$, by all $e_{\alpha}$ with $\alpha \in \Phi_{e}$, and by $e$. Let $h_{1}, \ldots, h_{l-1}$ be a basis of $\mathfrak{h}_{e}$, and let $\mathfrak{n}^{ \pm}(i)$ be the span of all $e_{\alpha}$ with $\alpha \in \Phi_{e, i}^{ \pm}$. Clearly, $\mathfrak{n}^{+}(0)$ and $\mathfrak{n}^{-}(0)$ are maximal nilpotent subalgebras of $\mathfrak{g}(0)^{\sharp}$. Let $\left\{x_{1}, \ldots, x_{t}\right\}$ and $\left\{y_{1}, \ldots, y_{t}\right\}$ be bases of $\mathfrak{n}^{+}(0)$ and $\mathfrak{n}^{-}(0)$ consisting of root vectors $e_{\alpha}$ with $\alpha \in \Phi$. Recall that the $z_{i}$ 's with $1 \leq i \leq 2 s$ are root vectors for $\mathfrak{h}$. For $1 \leq i \leq s$, set $u_{i}=\left[e, z_{i}\right]$ and $u_{i}^{*}=\left[e, z_{i}^{*}\right]$. It follows from our discussion in 4.1 that $u_{i}$ (resp. $u_{i}^{*}$ ) is a root vector for $\mathfrak{h}$ corresponding to the root $\beta+\gamma_{i} \in \Phi_{e, 1}^{-}$(resp. $\beta+\gamma_{i}^{*} \in \Phi_{e, 1}^{+}$). Furthermore, $\left\{u_{1}, \ldots, u_{s}, u_{1}^{*}, \ldots, u_{s}^{*}\right\}$ is a $\mathbb{k}$-basis of $\mathfrak{z}_{\chi}(1)$. 
Given a linear function $\lambda$ on $\mathfrak{h}_{e}$ and $c \in \mathbb{k}$ we denote by $J_{\lambda, c}$ the linear span in $H$ of all PBW monomials of the form

$$
\prod_{i=1}^{t} \Theta_{y_{i}}^{l_{i}} \cdot \prod_{i=1}^{s} \Theta_{u_{i}}^{m_{i}} \cdot \prod_{i=1}^{\ell-1}\left(\Theta_{h_{i}}-\lambda\left(h_{i}\right)\right)^{n_{i}} \cdot(C-c)^{n_{\ell}} \cdot \prod_{i=1}^{s} \Theta_{u_{i}^{*}}^{r_{i}} \cdot \prod_{i=1}^{t} \Theta_{x_{i}}^{q_{i}},
$$

where $\sum_{i=1}^{\ell} n_{i}+\sum_{i=1}^{t} r_{i}+\sum_{i=1}^{s} q_{i}>0$.

Lemma 7.1. The subspace $J_{\lambda, c}$ is a left ideal of the algebra $H$.

Proof. For $\mathbf{a}, \mathbf{b} \in \mathbb{Z}_{+}^{t}, \mathbf{c}, \mathbf{d} \in \mathbb{Z}_{+}^{s}, \mathbf{m} \in \mathbb{Z}_{+}^{\ell}$, set

$$
\Theta(\mathbf{a}, \mathbf{b}, \mathbf{c}, \mathbf{d}, \mathbf{m}):=\left(\prod_{i=1}^{t} \Theta_{y_{i}}^{a_{i}}\right)\left(\prod_{i=1}^{s} \Theta_{u_{i}}^{c_{i}}\right)\left(\prod_{i=1}^{\ell-1} \Theta_{h_{i}}^{m_{i}}\right) C^{m_{l}}\left(\prod_{i=1}^{s} \Theta_{u_{i}^{*}}^{d_{i}}\right)\left(\prod_{i=1}^{t} \Theta_{x_{i}}^{b_{i}}\right) .
$$

By [31, Theorem 4.6(ii)], the PBW monomials $\Theta(\mathbf{a}, \mathbf{b}, \mathbf{c}, \mathbf{d}, \mathbf{m})$ form a $\mathbb{k}$-basis of $H$. Note that $\operatorname{deg}_{e}(\Theta(\mathbf{a}, \mathbf{b}, \mathbf{c}, \mathbf{d}, \mathbf{m}))=4 m_{\ell}+3(|\mathbf{c}|+|\mathbf{d}|)+2(|\mathbf{a}|+|\mathbf{b}|)+2 \sum_{i=1}^{\ell-1} m_{i}$.

Since $C-c$ is central in $H$ we have $\Theta(\mathbf{a}, \mathbf{b}, \mathbf{c}, \mathbf{d}, \mathbf{m})(C-c) \in J_{\lambda, c}$. Relations (i) and (ii) of Theorem 6.1 imply that $\Theta(\mathbf{a}, \mathbf{b}, \mathbf{c}, \mathbf{d}, \mathbf{m})\left(\Theta_{h_{i}}-\lambda\left(h_{i}\right)\right) \in J_{\lambda, c}$ for $1 \leq i \leq$ $\ell-1$. Since $\Theta\left(\mathfrak{n}^{+}(0)\right)$ is a Lie subalgebra of $\Theta(\mathfrak{z} \chi(0))$, by Theorem 6.1, we also have $\Theta(\mathbf{a}, \mathbf{b}, \mathbf{c}, \mathbf{d}, \mathbf{m}) \cdot \Theta_{e_{\alpha}} \in J_{\lambda, c}$ for all $\alpha \in \Phi_{e, 0}^{+}$.

It remains to show that $\Theta(\mathbf{a}, \mathbf{b}, \mathbf{c}, \mathbf{d}, \mathbf{m}) \cdot \Theta_{u_{i}^{*}} \in J_{\lambda, c}$ for all $i \leq s$. We shall use induction on $\operatorname{deg}_{e}(\Theta(\mathbf{a}, \mathbf{b}, \mathbf{c}, \mathbf{d}, \mathbf{m}))$, so assume from now on that $\operatorname{deg}_{e}(\Theta(\mathbf{a}, \mathbf{b}, \mathbf{c}, \mathbf{d}, \mathbf{m}))=N$ and $H^{k} \cdot \Theta_{u_{i}^{*}} \in J_{\lambda, c}$ for all $i \leq s$ and all $k<N$. First note that the span of $u_{1}^{*}, \ldots, u_{s}^{*}$ equals $\mathfrak{n}^{+}(1)$, hence is stable under the adjoint action of $\mathfrak{n}^{+}(0)$. Since we have already established that $H \cdot \Theta_{e_{\alpha}} \in J_{\lambda, c}$ for all $\alpha \in \Phi_{e, 0}$, relation (ii) of Theorem 6.1 yields

$$
\Theta(\mathbf{a}, \mathbf{b}, \mathbf{c}, \mathbf{d}, \mathbf{m}) \cdot \Theta_{u_{i}^{*}} \in \Theta(\mathbf{a}, \mathbf{b}, \mathbf{c}, \mathbf{0}, \mathbf{m}) \cdot \Theta\left(\mathfrak{n}^{+}(1)\right)+J_{\lambda, c} .
$$

Thus we may assume that $\mathbf{d}=\mathbf{0}$. If $b_{j}=0$ for all $j>i$, then $\Theta(\mathbf{a}, \mathbf{b}, \mathbf{c}, \mathbf{0}, \mathbf{m}) \cdot \Theta_{u_{i}^{*}}=$ $\Theta\left(\mathbf{a}, \mathbf{b}+\mathbf{e}_{i}, \mathbf{c}, \mathbf{0}, \mathbf{m}\right) \in J_{\lambda, c}$. So suppose $\mathbf{b}=\left(b_{1}, \ldots, b_{k}, 0, \ldots, 0\right)$ where $b_{k}>0$ and $k>i$. Then in view of [31, Theorem 4.6(iv)] and our induction assumption we have

$$
\begin{aligned}
\Theta(\mathbf{a}, \mathbf{b}, \mathbf{c}, \mathbf{0}, \mathbf{m}) \cdot \Theta_{u_{i}^{*}} \in & \Theta\left(\mathbf{a}, \mathbf{b}+\mathbf{e}_{i}, \mathbf{c}, \mathbf{0}, \mathbf{m}\right)+\Theta\left(\mathbf{a}, \mathbf{b}-\mathbf{e}_{k}, \mathbf{c}, \mathbf{0}, \mathbf{m}\right)\left[\Theta_{u_{k}^{*}}, \Theta_{u_{i}^{*}}\right] \\
& +H^{N-2} \cdot \Theta_{u_{k}^{*}} \subseteq \Theta\left(\mathbf{a}, \mathbf{b}-\mathbf{e}_{k}, \mathbf{c}, \mathbf{0}, \mathbf{m}\right)\left[\Theta_{u_{k}^{*}}, \Theta_{u_{i}^{*}}\right]+J_{\lambda, c} .
\end{aligned}
$$

Since $\left(f,\left[u_{k}^{*}, u_{i}^{*}\right]\right)=0$, Theorem 6.1 shows that

$$
\left[\Theta_{u_{k}^{*}}, \Theta_{u_{i}^{*}}\right]=\frac{1}{2} \sum_{j=1}^{2 s}\left(\Theta_{\left[u_{k}^{*}, z_{j}\right]^{\sharp}} \Theta_{\left[u_{i}^{*}, z_{j}^{*}\right]^{\sharp}}+\Theta_{\left[u_{i}^{*}, z_{j}^{*}\right]^{\sharp}} \Theta_{\left[u_{k}^{*}, z_{j}\right]^{\sharp}}\right) \in \sum_{\alpha \in \Phi_{e, 0}^{+}} H \cdot \Theta_{e_{\alpha}}
$$

(one should take into account that $\left[u_{i}^{*}, z_{j}^{*}\right],\left[\left[u_{i}^{*}, z_{j}^{*}\right]^{\sharp},\left[u_{k}^{*}, z_{j}\right]^{\sharp}\right] \in \bigcup_{\alpha \in \Phi_{e, 0}^{+}} \mathbb{k} e_{\alpha}$ for all $j \leq s)$. So $\Theta(\mathbf{a}, \mathbf{b}, \mathbf{c}, \mathbf{0}, \mathbf{m}) \cdot \Theta_{u_{i}^{*}} \in J_{\lambda, c}$, and the result follows by induction on $N$. 
7.2. Put $Z_{H}(\lambda, c):=H / J_{\lambda, c}$ and let $v_{0}$ denote the image of 1 in $Z_{H}(\lambda, c)$. Clearly, $Z_{H}(\lambda, c)$ is a cyclic $H$-module generated by $v_{0}$. We call $Z_{H}(\lambda, c)$ the Verma module of level c corresponding to $\lambda$. By Lemma 7.1 , the vectors

$$
\left\{\Theta_{y_{1}}^{l_{1}} \ldots \Theta_{y_{t}}^{l_{t}} \Theta_{u_{1}}^{m_{1}} \ldots \Theta_{u_{s}}^{m_{s}}\left(v_{0}\right) \mid l_{1}, \ldots, l_{t}, m_{1}, \ldots, m_{s} \in \mathbb{Z}_{+}\right\}
$$

form a $\mathbb{k}$-basis of the Verma module $Z_{H}(\lambda, c)$. Let $Z_{H}^{+}(\lambda, c)$ denote the $\mathbb{k}$-span of all $\left\{\Theta_{y_{1}}^{l_{1}} \ldots \Theta_{y_{t}}^{l_{t}} \Theta_{u_{1}}^{m_{1}} \ldots \Theta_{u_{s}}^{m_{s}}\left(v_{0}\right)\right.$ with $\sum_{i} l_{i}+\sum_{i} m_{i}>0$. Let $Z_{H}^{\max }(\lambda, c)$ denote the sum of all $H$-submodules of $Z_{H}(\lambda, c)$ contained in $Z_{H}^{+}(\lambda, c)$, and put

$$
L_{H}(\lambda, c):=Z_{H}(\lambda, c) / Z_{H}^{\max }(\lambda, c) .
$$

Proposition 7.1. The following are true:

(i) $Z_{H}^{\max }(\lambda, c)$ is a unique maximal submodule of the Verma module $Z_{H}(\lambda, c)$ and hence $L_{H}(\lambda, c)$ is a simple $H$-module.

(ii) The simple $H$-modules $L_{H}(\lambda, c)$ and $L_{H}\left(\lambda^{\prime}, c^{\prime}\right)$ are isomorphic if and only if $\lambda=\lambda^{\prime}$ and $c=c^{\prime}$.

(iii) Any finite-dimensional simple $H$-module is isomorphic to one of the modules $L_{H}(\lambda, c)$ with $\lambda \in \mathfrak{h}_{e}^{*}$ satisfying $\lambda\left(h_{\alpha}\right) \in \mathbb{Z}_{+}$for all $\alpha \in \Phi_{e, 0}^{+}$. Furthermore, if $\mathfrak{g}$ is not of type A then $c$ is a rational number.

Proof. (a) For a root $\alpha=\sum_{i=1}^{\ell} n_{i} \alpha_{i}$ in $\Phi$ we put

$$
\mathrm{ht}_{\beta}(\alpha):=\sum_{\alpha_{i} \neq \beta} n_{i} .
$$

Clearly, $\mathrm{ht}_{\beta}(\alpha)=0$ if and only if $\alpha= \pm \beta$. As all derivations of $\mathfrak{g}$ are inner, there is a unique $h_{0} \in \mathfrak{h}$ such that $\left[h_{0}, e_{\alpha}\right]=\mathrm{ht}_{\beta}(\alpha) e_{\alpha}$ for all $\alpha \in \Phi$. As $\left[h_{0}, e_{ \pm \beta}\right]=0$ we see that $h_{0} \in \mathfrak{h}_{e}$. Clearly, $\Theta_{h_{0}}\left(v_{0}\right)=\lambda\left(h_{0}\right) v_{0}$ and $Z_{H}(\lambda, c)=\mathbb{k} v_{0} \oplus Z_{H}^{+}(\lambda, c)$. Since all $y_{i}$ and $z_{i}$ are root vectors for $\mathfrak{h}$ corresponding to negative roots different from $-\beta$, it follows from Theorem 6.1 that the subspace $Z_{H}^{+}(\lambda, c)$ decomposes into eigenspaces for $\Theta_{h_{0}}$ and the eigenvalues of $\Theta_{h_{0}}$ on $Z_{H}^{+}(\lambda, c)$ are of the form $\lambda\left(h_{0}\right)-k$ where $k$ is a positive integer.

Let $V$ be a nonzero $H$-submodule of $Z_{H}(\lambda, c)$. If $V \nsubseteq Z_{H}^{+}(\lambda, c)$, the above discussion shows that $v_{0} \in V$. But then $V=Z_{H}(\lambda, c)$. Thus any proper submodule of $Z_{H}(\lambda, c)$ is contained in $Z_{H}^{+}(\lambda, c)$. As a consequence, $Z_{H}^{\max }(\lambda, c)$ is a unique maximal submodule of $Z_{H}(\lambda, c)$, proving (i).

(b) It follows from part (a) that each $H$-module $L_{H}(\lambda, c)$ decomposes into eigenspaces for $\Theta_{h_{0}}$, the eigenvalues of $\Theta_{h_{0}}$ on $L_{H}(\lambda, c)$ lie in the set $\lambda\left(h_{0}\right)-\mathbb{Z}_{+}$, and the eigenspace $L_{H}(\lambda, c)_{\lambda\left(h_{0}\right)}$ is spanned by $v_{0}$. If $L_{H}(\lambda, c) \cong L_{H}\left(\lambda^{\prime}, c^{\prime}\right)$ as $H$-modules then it must be that $\lambda\left(h_{0}\right) \in \lambda^{\prime}\left(h_{0}\right)-\mathbb{Z}_{+}$and $\lambda^{\prime}\left(h_{0}\right) \in \lambda\left(h_{0}\right)-\mathbb{Z}_{+}$. This implies that $\lambda\left(h_{0}\right)=$ $\lambda^{\prime}\left(h_{0}\right)$ and $L_{H}(\lambda, c)_{\lambda\left(h_{0}\right)} \cong L_{H}\left(\lambda^{\prime}, c^{\prime}\right)_{\lambda^{\prime}\left(h_{0}\right)}$ as modules over the commutative subalgebra $\Theta\left(\mathfrak{h}_{e}\right) \oplus \mathbb{k} C$ of $H$. But then $\lambda=\lambda^{\prime}$ and $c=c^{\prime}$, hence (ii).

(c) Let $M$ be a finite-dimensional simple $H$-module. Then $C \in Z(H)$ acts on $M$ as $c$ id for some $c \in \mathbb{k}$. Since $\Theta\left(\mathfrak{h}_{e}\right)$ is abelian, $M$ contains at least one weight subspace for $\Theta\left(\mathfrak{h}_{e}\right)$. 
From Theorem 6.1 it follows that the direct sum $\bigoplus_{\mu \in \mathfrak{h}_{e}^{*}} M_{\mu}$ of all weight subspaces of $M$ is an $H$-submodule of $M$. Hence $M$ decomposes into weight spaces relative to $\Theta\left(\mathfrak{h}_{e}\right)$. Note that any linear function on $\mathfrak{h}$ vanishing on $\mathfrak{h}_{e}$ is a scalar multiple of $\beta$. Since $\beta$ is a simple root, any sum of roots from $\Phi_{e}^{+}$restricts to a nonzero function on $\mathfrak{h}_{e}$. But then the relation

$$
\phi \geq \psi \Leftrightarrow \phi=\psi+\left(\sum_{\gamma \in \Phi_{e}^{+}} r_{\gamma} \gamma\right)_{\mid \mathfrak{h}_{e}}, \quad r_{\gamma} \in \mathbb{Z}_{+}, \quad\left(\forall \phi, \psi \in \mathfrak{h}_{e}^{*}\right)
$$

is a partial ordering on $\mathfrak{h}_{e}^{*}$. Since the set of $\Theta\left(\mathfrak{h}_{e}\right)$-weights of $M$ is finite, it contains at least one maximal element with respect to this ordering, $\lambda$ say. Let $m$ be a nonzero vector in $M_{\lambda}$. Then $\Theta_{x_{i}}(m)=\Theta_{u_{i}^{*}}(m)=0$ for all admissible $i$. As a consequence, there exists a homomorphism of $H$-modules $\xi: Z_{H}(\lambda, c) \rightarrow M$ such that $\xi\left(v_{0}\right)=m$. The simplicity of $M$ implies that $\xi$ is surjective, while part (a) yields $\operatorname{Ker} \xi=Z_{H}^{\max }(\lambda, c)$. Restricting $M$ to the $\mathfrak{s l}_{2}$-triple $\left(\Theta_{e_{\alpha}}, \Theta_{h_{\alpha}}, \Theta_{e_{-\alpha}}\right) \subset H$ with $\alpha \in \Phi_{e, 0}$ it is easy to observe that $\lambda\left(h_{\alpha}\right) \in \mathbb{Z}_{+}$ for all $\alpha \in \Phi_{e, 0}^{+}$.

Finally, suppose $\mathfrak{g}$ is not of type A. Then $\mathfrak{z}_{\chi}(0)$ is a semisimple Lie algebra. By Weyl's theorem, $M$ is a completely reducible $\Theta\left(\mathfrak{z}_{\chi}(0)\right)$-module. Let $\mathfrak{g}_{\mathbb{Q}}$ be the $\mathbb{Q}$-form in $\mathfrak{g}$ spanned by the Chevalley system from 4.1 and $\mathfrak{z} \chi, \mathbb{Q}(i)=\mathfrak{g}_{\mathbb{Q}} \cap \mathfrak{z} \chi(i)$ where $i=0,1$. Choose $u, v \in \mathfrak{z}_{\chi}, \mathbb{Q}(1)$ with $(f,[u, v])=2$. Then $\left[u, z_{i}\right]^{\sharp},\left[v, z_{i}^{*}\right]^{\sharp} \in \mathfrak{z}_{\chi}, \mathbb{Q}(0)$ for all $i$. The highest weight theory implies that there is a $\mathbb{Q}$-form in $M$ stable under the action of $\Theta\left(\mathfrak{z}_{\chi}, \mathbb{Q}(0)\right)$. It follows that $\operatorname{tr}_{M}\left(\Theta_{\left[u, z_{i}\right]^{\sharp}} \Theta_{\left[v, z_{i}^{*}\right]^{\sharp}}\right) \in \mathbb{Q}$ for $1 \leq i \leq 2 s$. Since $\operatorname{tr}_{M}\left[\Theta_{u}, \Theta_{v}\right]=0$, Theorem 6.1 entails that $\left(c-c_{0}\right) \operatorname{dim} M \in \mathbb{Q}$. Since $c_{0} \in \mathbb{Q}$ by Theorem 6.1. we obtain $c \in \mathbb{Q}$.

7.3. To determine the composition factors of the Verma modules $Z_{H}(\lambda, c)$ with their multiplicities we are going to establish a link between these $H$-modules and the $\mathfrak{g}$-modules obtained by parabolic induction from Whittaker modules for $\mathfrak{s l}_{2}(\mathbb{k})$. The latter modules have been studied in much detail in $[26,28,1]$, and it is known that their composition multiplicities can be calculated by using the Kazhdan-Lusztig algorithm. We are going to rely on Skryabin's equivalence (3.1); the Kazhdan filtration of $H$ will play an important rôle too.

Let $\mathfrak{s}_{\beta}$ denote the subalgebra of $\mathfrak{g}$ spanned by $(e, h, f)=\left(e_{\beta}, h_{\beta}, f_{\beta}\right)$, and put

$$
\mathfrak{p}_{\beta}:=\mathfrak{s}_{\beta}+\mathfrak{h}+\sum_{\alpha \in \Phi^{+}} \mathbb{k} e_{\alpha}, \quad \mathfrak{n}_{\beta}:=\sum_{\alpha \in \Phi^{+} \backslash\{\beta\}} \mathbb{k} e_{\alpha}, \quad \widetilde{\mathfrak{s}}_{\beta}:=\mathfrak{h}_{e} \oplus \mathfrak{s} \beta .
$$

Clearly, $\mathfrak{p}_{\beta}=\widetilde{\mathfrak{s}}_{\beta} \oplus \mathfrak{n}_{\beta}$ is a parabolic subalgebra of $\mathfrak{g}$ with nilradical $\mathfrak{n}_{\beta}$ and $\widetilde{\mathfrak{s}}_{\beta}$ is a Levi subalgebra of $\mathfrak{p}_{\beta}$. Let $C_{\beta}=e f+f e+\frac{1}{2} h^{2}=2 e f+\frac{1}{2} h^{2}-h$ be the Casimir element of $U\left(\mathfrak{s}_{\beta}\right)$. Given $\lambda \in \mathfrak{h}_{e}^{*}$ and $c \in \mathbb{k}$ we denote by $I_{\beta}(\lambda, c)$ the left ideal of $U\left(\mathfrak{p}_{\beta}\right)$ generated by $f-1, C_{\beta}-c$, all $h-\lambda(h)$ with $h \in \mathfrak{h}_{e}$, and all $e_{\gamma}$ with $\gamma \in \Phi^{+} \backslash\{\beta\}$.

Define $Y(\lambda, c):=U\left(\mathfrak{p}_{\beta}\right) / I_{\beta}(\lambda, c)$, a $\mathfrak{p}_{\beta}$-module with the trivial action of $\mathfrak{n}_{\beta}$, and let $1_{\lambda, c}$ denote the image of 1 in $Y(\lambda, c)$. Since $f .1_{\lambda, c}=1_{\lambda, c}$, we have

$$
e .1_{\lambda, c}=\frac{1}{2}\left(C_{\beta}-\frac{1}{2} h^{2}+h\right) \cdot 1_{\lambda, c}=\left(-\frac{1}{4} h^{2}+\frac{1}{2} h+\frac{1}{2} c\right) .1_{\lambda, c} .
$$


Together with the PBW theorem this shows that the vectors $\left\{h^{k} \cdot 1_{\lambda, c} \mid k \in \mathbb{Z}_{+}\right\}$form a $\mathbb{k}$-basis of $Y(\lambda, c)$ (the independence of these vectors follows from the fact that $Y(\lambda, c)$ is infinite-dimensional). We mention for completeness that $Y(\lambda, c)$ is isomorphic to a Whittaker module for $\mathfrak{s}_{\beta} \cong \mathfrak{s l}_{2}(\mathbb{k})$.

The above discussion shows that the vectors

$$
m(\mathbf{i}, \mathbf{j}, \mathbf{k}, l):=z_{1}^{i_{1}} \cdots z_{s}^{i_{s}} \cdot y_{1}^{j_{1}} \cdots y_{t}^{j_{t}} \cdot u_{1}^{k_{1}} \cdots u_{s}^{k_{s}} \cdot h^{l}\left(1_{\lambda, c}\right)
$$

with $\mathbf{i}, \mathbf{k} \in \mathbb{Z}_{+}^{s}, \mathbf{j} \in \mathbb{Z}_{+}^{t}$, and $l \in \mathbb{Z}_{+}$form a $\mathbb{k}$-basis of the induced $\mathfrak{g}$-module

$$
M(\lambda, c):=U(\mathfrak{g}) \otimes_{U\left(\mathfrak{p}_{\beta}\right)} Y(\lambda, c)
$$

7.4. Recall from 4.1 that each $z_{i}^{*}$ with $1 \leq i \leq s$ is a root vector for $\mathfrak{h}$ corresponding to $\gamma_{i}^{*}=-\beta-\gamma_{i} \in \Phi^{+}$. Put $\delta=\frac{1}{2}\left(\gamma_{1}^{*}+\cdots+\gamma_{s}^{*}\right)$ and $\rho_{0}=\rho-2 \delta-(s+1) \beta=\sum_{\alpha \in \Phi_{e, 0}^{+}} \alpha$. Since the restriction of $(\cdot, \cdot)$ to $\mathfrak{h}_{e}$ is nondegenerate, for any $\eta \in \mathfrak{h}_{e}^{*}$ there exists a unique $t_{\eta} \in \mathfrak{h}_{e}$ such that $\varphi=\left(t_{\eta}, \cdot\right)$. Hence $(\cdot, \cdot)$ induces a nondegenerate bilinear form on $\mathfrak{h}_{e}^{*}$ via $(\mu, v):=\left(t_{\mu}, t_{\nu}\right)$ for all $\mu, v \in \mathfrak{h}_{e}^{*}$. Given a linear function $\varphi$ on $\mathfrak{h}$ we denote by $\bar{\varphi}$ the restriction of $\varphi$ to $\mathfrak{h}_{e}$.

Theorem 7.1. Each $\mathfrak{g}$-module $M(\lambda, c)$ is an object of the category $\mathrm{C}$. Furthermore, $\mathrm{Wh}(M(\lambda, c)) \cong Z_{H}(\lambda+\bar{\delta}, c+(\lambda+2 \bar{\rho}, \lambda))$ as $H$-modules.

Proof. Put $M:=M(\lambda, c)$, and let $M_{0}$ (resp. $\left.M_{1}\right)$ denote the $\mathbb{k}$-span of all $m(\mathbf{i}, \mathbf{j}, \mathbf{k}, l)$ in $M$ with $|\mathbf{i}|+l=0$ (resp. $|\mathbf{i}|+l>0$ ). Clearly, $M=M_{0} \oplus M_{1}$ as vector spaces. Let pr: $M=M_{0} \oplus M_{1} \rightarrow M_{0}$ denote the first projection.

If $|\mathbf{i}|+2|\mathbf{j}|+3|\mathbf{k}|+2 l=k$, we say that $m(\mathbf{i}, \mathbf{j}, \mathbf{k}, l)$ has Kazhdan degree $k$. Let $M^{k}$ denote the $\mathbb{k}$-span in $M$ of all $m(\mathbf{i}, \mathbf{j}, \mathbf{k}, l)$ of Kazhdan degree $\leq k$. Then $\left\{M^{k} \mid k \in \mathbb{Z}_{+}\right\}$ is an increasing filtration in $M$ and $M^{0}=\mathbb{k} 1_{\lambda, c}$. Taking $U(\mathfrak{g})$ with its Kazhdan filtration (as defined in [10] for example) we can thus regard $M$ as a filtered $U(\mathfrak{g})$-module.

Let $z=\lambda f+\sum_{i=1}^{s} \mu_{i} z_{i}^{*} \in \mathfrak{m}_{\chi}$ where $\lambda, \mu_{i} \in \mathbb{k}$. Since $z_{i}^{*} \in \mathfrak{n}_{\beta}$ for $1 \leq i \leq s$ and $f .1_{\lambda, c}=1_{\lambda, c}$, we have $z \cdot 1_{\lambda, c}=\lambda \cdot 1_{\lambda, c}=\chi(z) \cdot 1_{\lambda, c}$. Since $z$ acts locally nilpotently on $U(\mathfrak{g})$, we deduce that $z-\chi(z)$ acts locally nilpotently on $M$ for all $z \in \mathfrak{m}_{\chi}$. As a consequence, $M$ is an object of $\mathrm{C}_{\chi}$. By our discussion in 3.1. Wh $(M) \neq 0$, the algebra $H$ acts on $M$, and $M \cong Q_{\chi} \otimes_{H} \mathrm{Wh}(M)$ as $\mathfrak{g}$-modules.

Now observe that

$$
z_{k}^{*} \cdot m(\mathbf{i}, \mathbf{j}, \mathbf{k}, l) \in i_{k} \cdot m\left(\mathbf{i}-\mathbf{e}_{k}, \mathbf{j}, \mathbf{k}, l\right)+\operatorname{span}\left\{m\left(\mathbf{i}^{\prime}, \mathbf{j}^{\prime}, \mathbf{k}^{\prime}, l^{\prime}\right)|| \mathbf{i}^{\prime}|\geq| \mathbf{i} \mid\right\}
$$

for all $k \leq s$, and

$$
(f-1) \cdot m(\mathbf{i}, \mathbf{j}, \mathbf{k}, l) \in 2^{l} \cdot m(\mathbf{i}, \mathbf{j}, \mathbf{k}, 0)+\operatorname{span}\left\{m\left(\mathbf{i}^{\prime}, \mathbf{j}^{\prime}, \mathbf{k}^{\prime}, l^{\prime}\right) \mid l^{\prime}>0\right\} \quad \text { when } l>0 .
$$

From this it is immediate that the map pr: $\mathrm{Wh}(M) \rightarrow M_{0}$ is injective. 
Note that $1_{\lambda, c} \in \mathrm{Wh}(M)$, and for all $h \in \mathfrak{h}_{e}$ we have

$$
\begin{aligned}
\Theta_{h}\left(1_{\lambda, c}\right) & =\left(h+\frac{1}{2} \sum_{i=1}^{2 s}\left[h, z_{i}^{*}\right] z_{i}\right)\left(1_{\lambda, c}\right)=\left(h+\frac{1}{2} \sum_{i=1}^{s}\left[\left[h, z_{i}^{*}\right], z_{i}\right]\right)\left(1_{\lambda, c}\right) \\
& =\left(\lambda(h)+\frac{1}{2} \sum_{i=1}^{s} \gamma_{i}^{*}(h) f\right) \cdot 1_{\chi, c}=(\lambda+\delta)(h) \cdot 1_{\chi, c} .
\end{aligned}
$$

Suppose $x \in \mathfrak{z}_{\chi}(0)$ is a root vector for $\mathfrak{h}$ corresponding to root $\gamma \in \Phi_{e, 0}^{+}$. Then $x \in \mathfrak{n}_{\beta}$ and $\left[\left[x, z_{i}^{*}\right], z_{i}\right] \in \mathfrak{n}_{\beta}$ for all $i \leq s$. Therefore,

$$
\Theta_{x}\left(1_{\lambda, c}\right)=\left(x+\frac{1}{2} \sum_{i=1}^{2 s}\left[x, z_{i}^{*}\right] z_{i}\right)\left(1_{\lambda, c}\right)=\left(h+\frac{1}{2} \sum_{i=1}^{s}\left[\left[x, z_{i}^{*}\right], z_{i}\right]\right)\left(1_{\lambda, c}\right)=0 .
$$

Recall from (2.5) that for any positive root vector $u \in \mathfrak{z}_{\chi}(1)$ we have

$$
z_{u}=-\frac{1}{3} \sum_{i=1}^{2 s}\left\langle z_{i}^{*},\left[u,\left[z_{i}, z_{i}^{*}\right]\right]\right\rangle z_{i}=\frac{1}{3} \sum_{i=1}^{2 s}\left\langle z_{i}^{*},[u, f]\right\rangle z_{i} \in \mathfrak{n}_{\beta} .
$$

This implies that

$$
\begin{aligned}
\Theta_{u}\left(1_{\lambda, c}\right) & =\left(u+\sum_{i=1}^{2 s}\left[u, z_{i}^{*}\right] z_{i}+\frac{1}{3} \sum_{i, j=1}^{2 s}\left[u z_{i}^{*} z_{j}^{*}\right] z_{j} z_{i}+z_{u}\right)\left(1_{\lambda, c}\right) \\
& =\left(\sum_{i=1}^{s}\left[\left[u, z_{i}^{*}\right], z_{i}\right]+\frac{1}{3} \sum_{i, j=1}^{s}\left[u z_{i}^{*} z_{j}^{*}\right] z_{j} z_{i}+\frac{1}{3} \sum_{i=1}^{s} \sum_{j=s+1}^{2 s}\left[u z_{i}^{*} z_{j}^{*}\right] z_{j} z_{i}\right)\left(1_{\lambda, c}\right) \\
& =\frac{1}{3}\left(\sum_{i, j=1}^{s}\left[\left[u z_{i}^{*} z_{j}^{*}\right], z_{j}\right] z_{i}+\sum_{i, j=1}^{s} z_{j}\left[\left[u z_{i}^{*} z_{j}^{*}\right], z_{i}\right]-\sum_{i=1}^{s}\left[u z_{i}^{*} z_{i}\right]\left[z_{i}^{*}, z_{i}\right]\right)\left(1_{\lambda, c}\right) \\
& =\frac{1}{3}\left(\sum_{i, j=1}^{s}\left[\left[\left[u z_{i}^{*} z_{j}^{*}\right], z_{j}\right], z_{i}\right]-\sum_{i=1}^{s}\left[u z_{i}^{*} z_{i}\right] f\right)\left(1_{\lambda, c}\right) \in \mathfrak{n}_{\beta} \cdot 1_{\lambda, c}=0 .
\end{aligned}
$$

Therefore, $\Theta_{u_{i}^{*}}\left(1_{\lambda, c}\right)=0$ for all $i \leq s$. Our discussion in 4.4 shows that

$$
\begin{aligned}
C\left(1_{\lambda, c}\right) & =\left(2 e+\frac{h^{2}}{2}-(s+1) h+C_{0}+2 \sum_{i=1}^{2 s}\left[e, z_{i}^{*}\right] z_{i}\right)\left(1_{\lambda, c}\right) \\
& =\left(C_{\beta}-s h+C_{0}+2 \sum_{i=1}^{s}\left[\left[e, z_{i}^{*}\right], z_{i}\right]\right)\left(1_{\lambda, c}\right) .
\end{aligned}
$$

As $\left[\left[\left[e, z_{i}^{*}\right], z_{i}\right], f\right]=\left[\left[[e, f], z_{i}^{*}\right], z_{i}\right]=\left[\left[h, z_{i}^{*}\right], z_{i}\right]=-f$ we have $\left[\left[e, z_{i}^{*}\right], z_{i}\right]-\frac{1}{2} h$ $\in \mathfrak{h}_{e}$ for all $i \leq s$. Let $x$ be an arbitrary element in $\mathfrak{h}_{e}$. Then $(x, h)=0, \beta(x)=0$, and

$$
\left.\left(x,\left[\left[e, z_{i}^{*}\right], z_{i}\right]-\frac{1}{2} h\right)=\left(\left[x,\left[e, z_{i}^{*}\right]\right], z_{i}\right]\right)=\gamma_{i}^{*}(x)\left(\left[e, z_{i}^{*}\right], z_{i}\right)=\gamma_{i}^{*}(x),
$$


that is, $\left[\left[e, z_{i}^{*}\right], z_{i}\right]-\frac{1}{2} h=t_{\bar{\gamma}_{i}^{*}}$ for all $i \leq s$; see our discussion at the beginning of this subsection. But then

$$
\left(2 \sum_{i=1}^{s}\left[\left[e, z_{i}^{*}\right], z_{i}\right]-s h\right)\left(1_{\lambda, c}\right)=\left(2 \sum_{i=1}^{s} t_{\bar{\gamma}_{i}^{*}}\right)\left(1_{\lambda, c}\right)=4(\lambda, \bar{\delta}) \cdot 1_{\lambda, c} .
$$

Since $C_{0}=\sum a_{i} b_{i}$ is a Casimir element of $U\left(\mathfrak{z}_{\chi}(0)\right)$ and all positive root vectors in $\mathfrak{z}_{\chi}(0)$ annihilate $1_{\lambda, c}$, it is straightforward to see that $C_{0}\left(1_{\lambda, c}\right)=\left(\lambda, \lambda+2 \bar{\rho}_{0}\right) \cdot 1_{\lambda, c}$. In conjunction with (7.4.1) and (7.4.2) this yields

$$
C\left(1_{\lambda, c}\right)=\left(c+\left(\lambda, \lambda+2 \bar{\rho}_{0}\right)+4(\lambda, \bar{\delta})\right) \cdot 1_{\lambda, c}=(c+(\lambda, \lambda+2 \bar{\rho})) \cdot 1_{\lambda, c} .
$$

Put $\lambda^{\prime}:=\lambda+\bar{\delta}$ and $c^{\prime}:=c+(\lambda, \lambda+2 \bar{\rho})$. Let $V_{0}$ denote the $H$-submodule of $M$ generated by $1_{\lambda, c}$. The above discussion shows that the left ideal $J_{\lambda^{\prime}, c^{\prime}}$ of $H$ annihilates $1_{\lambda, c}$. Therefore, $V_{0}$ is a homomorphic image of the Verma module $Z_{H}\left(\lambda^{\prime}, c^{\prime}\right)$.

We claim that the restriction of pr: $M \rightarrow M_{0}$ to $V_{0}$ is surjective. Recall that $M_{0}$ is spanned by all $m(\mathbf{0}, \mathbf{j}, \mathbf{k}, 0)$ with $\mathbf{j} \in \mathbb{Z}_{+}^{t}$ and $\mathbf{k} \in \mathbb{Z}_{+}^{s}$. Clearly, $m(\mathbf{0}, \mathbf{0}, \mathbf{0}, 0)=1_{\lambda, c} \in$ $\operatorname{pr}\left(V_{0}\right)$. Assume that all vectors $m(\mathbf{0}, \mathbf{j}, \mathbf{k}, 0)$ of Kazhdan degree $2|\mathbf{j}|+3|\mathbf{k}|<n$ are in $\operatorname{pr}\left(V_{0}\right)$. Now let $m(\mathbf{0}, \mathbf{a}, \mathbf{b}, 0) \in M_{0}$ be such that $2|\mathbf{a}|+3|\mathbf{b}|=n$ and $|\mathbf{a}|+|\mathbf{b}|=k$, and denote by $M_{n, k}$ the span of all $m(\mathbf{i}, \mathbf{j}, \mathbf{k}, l)$ of Kazhdan degree $n$ with $|\mathbf{i}|+|\mathbf{j}|+|\mathbf{k}|+l>k$. Assume that all vectors $m(\mathbf{0}, \mathbf{j}, \mathbf{k}, 0)$ of Kazhdan degree $n$ with $|\mathbf{j}|+|\mathbf{k}|>k$ are in $\operatorname{pr}\left(V_{0}\right)$. Since $M$ is a filtered $U(\mathfrak{g})$-module, it follows from Lemmas 2.3 and 2.5 that

$$
\Theta_{y_{1}}^{a_{1}} \cdots \Theta_{y_{t}}^{a_{t}} \Theta_{u_{1}}^{b_{1}} \cdots \Theta_{u_{s}}^{b_{s}}\left(1_{\lambda, c}\right) \in m(\mathbf{0}, \mathbf{a}, \mathbf{b}, 0)+M_{n, k}+M^{n-1} .
$$

In view of our assumptions on $n$ and $k$ we get $m(\mathbf{0}, \mathbf{a}, \mathbf{b}, 0) \in \operatorname{pr}\left(V_{0}+M^{n-1}+M_{n, k}\right)=$ $\operatorname{pr}\left(V_{0}\right)$. Our claim now follows by double induction on $n$ and $k$. Since we have already established that $\mathrm{pr}: \mathrm{Wh}(M) \rightarrow M_{0}$ is injective, this yields $\mathrm{Wh}(M)=V_{0}$.

Using 7.4 .3 it is easy to observe that the vectors $\Theta_{y_{1}}^{a_{1}} \ldots \Theta_{y_{t}}^{a_{t}} \Theta_{u_{1}}^{b_{1}} \ldots \Theta_{u_{s}}^{b_{s}}\left(1_{\lambda, c}\right)$ with $\mathbf{a} \in \mathbb{Z}_{+}^{t}$ and $\mathbf{b} \in \mathbb{Z}_{+}^{s}$ are linearly independent over $\mathbb{k}$. Hence it follows from Lemma 7.1 and our discussion at the beginning of 7.2 that $V_{0} \cong Z_{H}\left(\lambda^{\prime}, c^{\prime}\right)$ as $H$-modules.

Remark 7.1. Combined with Skryabin's equivalence and the main results of MiličićSoergel [27] and Backelin [1], Theorem 7.1 implies that the composition multiplicities of the Verma modules $Z_{H}(\lambda, c)$ can be computed with the help of inverse parabolic Kazhdan-Lusztig polynomials associated with the pair $\left(W,\left\langle s_{\beta}\right\rangle\right)$. This confirms in the minimal nilpotent case the Kazhdan-Lusztig conjecture for finite $\mathcal{W}$-algebras as formulated by de Vos and van Driel in [8]. Recall that our construction of $H_{\chi}$ is a special instance of quantum Hamiltonian reduction where the constraints imposed are read off from the $\mathfrak{s l}_{2}$-triple $\left(e_{\beta}, h_{\beta}, e_{-\beta}\right)$. In the physics literature the algebra $H$ appears undercover under the name of a finite $\mathcal{W}$-algebra associated with the minimal embedding $\mathfrak{s l}_{2}(\mathbb{k}) \hookrightarrow \mathfrak{g}$.

Remark 7.2. It would be interesting to relate the (Kazhdan) filtered algebra $H$ to the Becchi-Rouet-Stora-Tyutin (BRST) quantisation of the Poisson algebra gr $H$. We recall that the Poisson structure on gr $H$ is determined in [10]. It would be important for the characteristic $p$ theory to determine all $(\lambda, c) \in \mathfrak{h}_{e}^{*} \times \mathbb{k}$ such that the simple $H$-module $L_{H}(\lambda, c)$ is finite-dimensional; see Proposition 7.1 (iii). 
Given $t \in \mathbb{k}$ we let $H_{t}$ denote the factor algebra $H /(C-t)$ where $(C-t)$ is the two-sided ideal of $H$ generated by the central element $C-t$. It is clear from the definition that each $L(\lambda, t)$ is an $H_{t}$-module.

Corollary 7.1. If $\mathfrak{g}$ is of type $\mathrm{C}_{n}$ or $\mathrm{G}_{2}$, then any finite-dimensional $H_{t}$-module is completely reducible.

Proof. It suffices to show that $\operatorname{Ext}_{H_{t}}^{1}(M, N)=0$ for any two finite-dimensional simple $H_{t}$-modules $M$ and $N$. If the $H_{t}$-modules $M$ and $N$ are not isomorphic, then they have distinct central characters; see Theorem 6.2 Thus it remains to show that $\operatorname{Ext}_{H_{t}}^{1}(M, M)=0$. By Proposition 7.1(iii), $M \cong L_{H}(\lambda, t)$ for some $\lambda \in \mathfrak{h}_{e}^{*}$. Let $V$ be a finite-dimensional $H_{t}$-module containing $M$ as a submodule and such that $V / M \cong M$ as $H_{t}$-modules. As $\Theta\left(\mathfrak{z}_{\chi}(0)\right)$ is a semisimple Lie subalgebra of $H$, Weyl's theorem shows that $V$ decomposes into weight spaces relative to $\Theta\left(\mathfrak{h}_{e}\right)$, say $V=\bigoplus_{\mu \in X(V)} V_{\mu}$. Moreover, the set of $\Theta\left(\mathfrak{h}_{e}\right)$-weights of $V$ coincides with that of $L(\lambda, c)$, showing that $\mu \leq \lambda$ for all $\mu \in X(V)$. It follows from our discussion in 7.2 that $\operatorname{dim} M_{\lambda}=1$ and $\operatorname{dim} V_{\lambda}=2$. Let $v \in V_{\lambda} \backslash M_{\lambda}$ and let $M^{\prime}$ denote the $H$-submodule of $V$ generated by $v$. By construction, the left ideal $J_{\lambda, t}$ of $H$ annihilates $v$, showing that $M^{\prime}$ is a homomorphic image of the Verma module $Z_{H}(\lambda, t)$. But then the $\Theta\left(\mathfrak{h}_{e}\right)$-weight space $M_{\lambda}^{\prime}$ is 1-dimensional, implying $\left(M \cap M^{\prime}\right)_{\lambda}=M_{\lambda} \cap M_{\lambda}^{\prime}=0$. Consequently, $M \cap M^{\prime}=0$. The irreducibility of $V / M$ now entails that $V=M \oplus M^{\prime}$ and $M^{\prime} \cong M$. Then $\operatorname{Ext}_{H_{t}}^{1}(M, M)=0$, completing the proof.

Acknowledgements. I would like to thank Jonathan Brundan, Victor Ginzburg, Anthony Joseph and Alexander Kleshchev for interesting discussions and e-mail correspondence. Some results of this work were announced in my talks at the AMS Summer Research Conference in Snowbird, Utah in July 2004, at the Oberwolfach meeting on enveloping algebras in March 2005, and at the Luminy conference "Geometry and Representations" in April 2005.

\section{References}

[1] Backelin, E.: Representation of the category $\mathcal{O}$ in Whittaker categories. Int. Math. Res. Not. 1997, no. 4, 153-172 Zbl 0974.17007 MR 1436773

[2] Borho, W., Brylinski, J.-L.: Differential operators on homogeneous spaces. I. Irreducibility of the associated variety for annihilators of irreducible modules. Invent. Math. 69, 437-476 (1982) Zbl 0504.22015 MR 0679767

[3] Borho, W., Kraft, H.: Über die Gelfand-Kirillov-Dimension. Math. Ann. 220, 1-24 (1976) Zbl 0306.17005 MR 0412240

[4] Bourbaki, N.: Groupes et algèbres de Lie. Chapitres IV, V, VI. Hermann, Paris, 1968 Zbl 0186.33001 MR 0240238

[5] Brundan, J., Kleshchev, A.: Shifted Yangians and finite $\mathcal{W}$-algebras. Adv. Math. 200, 136-195 ((2006)) Zbl 1083.17006 MR 2199632

[6] Carter, R. W.: Finite Groups of Lie Type, Conjugacy Classes and Complex Characters. Wiley, Chichester (1985) Zbl 0567.20023 MR 1266626

[7] Conze, N.: Algèbres d'opérateurs différentiels et quotients des algèbres enveloppantes. Bull. Soc. Math. France 102, 379-415 (1974) Zbl 0298.17012 MR 0374214 
[8] de Vos, K., van Driel, P.: The Kazhdan-Lusztig conjecture for finite $\mathcal{W}$-algebras. Lett. Math. Phys. 35, 333-344 (1995) Zbl 0838.17027 MR 1358298

[9] Dixmier, J.: Algèbres Enveloppantes. Gauthier-Villars, Paris (1974) Zbl 0308.17007 MR 0498737

[10] Gan, W. L., Ginzburg, V.: Quantization of Slodowy slices. Int. Math. Res. Not. 2002, no. 5, 243-255 Zbl 0989.17014 MR 1876934

[11] Gan, W. T.: Savin, G.: Uniqueness of Joseph ideal. Math. Res. Lett. 11, 589-598 (2004) Zbl pre02152362 MR 2106228

[12] Ginzburg, V.: On primitive ideals. Selecta Math. (N. S.) 9, 379-407 (2003) Zbl 1041.16016 MR 2006573

[13] Jantzen, J. C.: Einhüllende Algebren halbeinfacher Lie-Algebren. Ergeb. Math. Grenzgeb. 3, Springer, New York (1983) Zbl 0541.17001 MR 0804695

[14] Joseph, A.: The minimal orbit in a simple Lie algebra and its associated maximal ideal. Ann. Sci. École Norm. Sup. (4) 9, 1-29 (1976) Zbl 0346.17008 MR 0404366

[15] Joseph, A.: A generalization of the Gelfand-Kirillov conjecture. Amer. J. Math. 99, 11511165 (1977) Zbl 0378.17005 MR 0460397

[16] Joseph, A.: A preparation theorem for the primitive spectrum of a semisimple Lie algebra. J. Algebra 48, 241-289 (1977) Zbl 0405.17007 MR 0453829

[17] Joseph, A.: Goldie rank in the enveloping algebra of a semisimple Lie algebra, II. J. Algebra 65, 284-306 (1980) Zbl 0441.17004 MR 0585721

[18] Joseph, A.: On the associated variety of a primitive ideal. J. Algebra 93, 509-523 (1985) Zbl 0594.17009 MR 0786766

[19] Joseph, A.: Orbital varieties of the minimal nilpotent orbit. Ann. Sci. École Norm. Sup. (4) 31, 17-45 (1998) Zbl 0908.17002 MR 1604290

[20] Kac, V. G.: Wakimoto, M.: Quantum reduction and representation theory of superconformal algebras. Adv. Math. 185, 400-458 (2004) Zbl 1049.17025 MR 2060475

[21] Kashiwara, M., Tanisaki, T.: The characteristic cycles of holonomic systems on a flag manifold related to the Weyl group algebra. Invent. Math. 77, 185-198 (1984) Zbl 0611.22008 MR 0751138

[22] Kawanaka, N.: Generalized Gelfand-Graev representations and Ennola duality. In: Algebraic Groups and Related Topics, Adv. Stud. Pure Math. 6, North-Holland, 175-206 (1985) MR 0803335

[23] Khare, A.: Category $\mathcal{O}$ over a deformation of the symplectic oscillator algebra. J. Pure Appl. Algebra 195, 131-166 (2005) Zbl 1083.17003 MR 2108468

[24] Matumoto, H.: Whittaker vectors and associated varieties. Invent. Math. 89, 219-224 (1987) Zbl 0633.17006 MR 0892192

[25] McConnell, J. C., Robson, J. C.: Noncommutative Noetherian Rings. Pure Appl. Math., Wiley-Interscience, Chichester (1988) Zbl 0644.16008 MR 0934572

[26] McDowell, E.: On modules induced from Whittaker modules. J. Algebra 96, 161-177 (1985) Zbl 0584.17002 MR 0808846

[27] McNinch, G. J., Sommers, E.: Component groups of unipotent centralisers in good characteristic. J. Algebra 260, 323-337 (2003) Zbl 1026.20026 MR 1976698

[28] Miličić, D.: Soergel, W.: The composition series of modules induced from Whittaker modules. Comment. Math. Helv. 72, 503-520 (1997) Zbl 0956.17004 MR 1600134

[29] Mœglin, C.: Modules de Whittaker et idéaux primitifs complètement premiers dans les algèbres enveloppantes. C. R. Acad. Sci. Paris 303, 845-848 (1986) Zbl 0628.17007 MR 0870903

[30] Mœglin, C.: Idéaux complètement premiers de l'algèbre enveloppante de $\mathfrak{g l}_{n}(\mathbb{C})$. J. Algebra 106, 287-366 (1987) Zbl 0637.17005 MR 0880962 
[31] Premet, A.: Special transverse slices and their enveloping algebras. Adv. Math. 170, 1-55 (2002) (with an Appendix by S. Skryabin) Zbl 1005.17007 MR 1929302

[32] Premet, A.: Nilpotent orbits in good characteristic and the Kempf-Rousseau theory. J. Algebra 260, 338-366 (2003) Zbl 1020.20031 MR 1976699

[33] Ragoucy, E., Sorba, P.: Yangian realisations from finite $W$-algebras. Comm. Math. Phys. 203, 551-572 (1999) Zbl 0943.17009 MR 1700166

[34] Shock, R. C.: Polynomial rings over finite-dimensional rings. Pacific J. Math. 42, 251-257 (1972) Zbl 0213.04301 MR 0318201

[35] Sommers, E.: A generalisation of the Bala-Carter theorem for nilpotent orbits. Int. Math. Res. Not. 1998, no. 11, 539-562 Zbl 0909.22014 MR 1631769

[36] Vogan, D.: Associated varieties and unipotent representations. In: Harmonic Analysis on Reductive Groups, Progr. Math. 101, Birkhäuser, Boston, MA, 315-388 (1991) Zbl 0832.22019 MR 1168491 